\title{
THE RESIDUE CALCULUS IN SEVERAL COMPLEX VARIABLES
}

\author{
BY \\ GERALD LEONARD GORDON $\left({ }^{1}\right)$
}

\begin{abstract}
Let $W$ be a complex manifold and $V$ an analytic variety. Then homology classes in $W-V$ which bound in $V$, called the geometric residues, are studied. In fact, a long exact sequence analogous to the Thom-Gysin sequence for nonsingular $V$ is formed by a geometric construction. A geometric interpretation of the Leray spectral sequence of the inclusion of $W-V \subset V$ is also given.

If the complex codimension of $V$ is one, then one shows that each cohomology class of $W-V$ can be represented by a differential form of the type $\theta \wedge \lambda$ $+\eta$ where $\lambda$ is the kernel associated to $V$ and $\theta \mid V$ is the Poincaré residue of this class.
\end{abstract}

1. Introduction. Let $W$ be a complex manifold and $V$ a subvariety of codimension $q$ in $W$. Then let

$$
R_{p}(V)=\operatorname{kernel}\left\{H_{p}(W-V) \rightarrow H_{p}(W)\right\}
$$

and $R_{p}(V)$ are called the geometric $q$-residues of $V$.

In the classical case when $W$ is a Riemann surface $R_{1}(V)$ is generated by small circles $\gamma_{i}$ about the points $P_{i} \in V$ and the relation is given by $\Sigma \gamma_{i} \sim 0$ in case $W$ is compact. This is a special case of when $V$ is nonsingular and one looks at the Thom-Gysin sequence of the normal sphere bundle of $V$ in $W$ :

$$
\rightarrow H_{p+1}(W) \stackrel{I}{\rightarrow} H_{p-2 q+1}(V) \stackrel{\tau}{\rightarrow} H_{p}(W-V) \rightarrow H_{p}(W) \rightarrow
$$

where $\tau$ is tubes over cycles and $I$ is transverse intersection.

In $\$ 2$ we study $V$ in the case when $V$ has normal crossing, i.e., transverse intersection of nonsingular hypersurfaces. We construct $H_{p}(V)_{\Delta}$, a quotient group of a subgroup of the $p$-cycles of $V$, such that

\section{COROLlaRy 2.14. The following is exact}

Presented to the Society, January 24, 1969; received by the editors March 9, 1971 and, in revised form, July 10, 1974.

AMS (MOS) subject classifications (1970). Primary 14C30, 14E15, 32A25, 32C10, $32 \mathrm{C30}, 53 \mathrm{C30}$.

Key words and phrases. Residues, analytic varieties, Thom-Gysin sequence, Whitney stratification, tubular neighborhoods, normal crossings, Poincaré residue operator, poles of order one.

(1) This research was partially supported by NSF Grant GP 21058. 


$$
\cdots \rightarrow H_{p+1}(W) \stackrel{I}{\rightarrow} H_{p-1}(V)_{\Delta} \stackrel{\tau}{\rightarrow} H_{p}(W-V) \rightarrow H_{p}(W) \rightarrow \cdots
$$

where $\tau$ will be induced from the nonsingular hypersurfaces.

The proof is geometric. In $\S 3$ it is shown that if one can resolve singularities via monoidal transforms with nonsingular centers, then it suffices to have normal crossings to study $R_{p}(V)$.

In $\S 4$ we consider the general case of $V$, a subvariety of codimension $q$ in $W$. There a tubular neighborhood $\tau(V) \subset W-V$ of $V$ in $W$ is constructed such that

COROLlaRY 4.17. The following is exact

$$
\cdots \rightarrow H_{p+1}(W) \rightarrow H_{p-2 q+1}(V)_{\Delta} \stackrel{\tau}{\rightarrow} H_{p}(W-V) \rightarrow H_{p}(W) \rightarrow \cdots
$$

where $\tau$ is the tubes over cycles via this tubular neighborhood and $H_{p-2 q+1}(V)_{\Delta}$ is a quotient group of a subgroup of the $(p-2 q+1)$-cycles of $V$.

The proof is geometric and uses Whitney work on stratifications to construct the tubular neighborhood. At the end of the section, the spectral sequence induced from the inclusion map $W-V \rightarrow W$ is studied and one has

$$
E_{2}^{p, q} \Rightarrow H^{p+q}(W-V)
$$

with

$$
E_{2}^{p, 0} \Rightarrow \text { image }\left\{H^{p}(W) \rightarrow H^{p}(W-V)\right\}
$$

and

$$
E_{2}^{p-q, q} \Rightarrow \operatorname{cokernel}\left\{H^{p}(W) \rightarrow H^{p}(W-V)\right\}
$$

(i.e., $R_{p}(V)$ ) for $q>1$. The $d_{2}$-term of the spectral sequence is essentially the Gysin map and the geometric results of this section are an investigation of this map.

We note here that Corollary 4.17 is true if $V$ satisfies Whitney condition A and $W$ is a differentiable manifold. In particular if $V$ has a regular neighborhood, e.g. $V$ is a polyhedron.

If we take coefficients in $\mathbf{C}$, rather than $Z$, and let $q=1$, then by dualizing Corollary 4.17 we get

$$
H^{p}(W-V) \stackrel{R}{\rightarrow} H^{p-1}(V)_{\Delta}=\operatorname{Hom}\left(H_{p-1}(V)_{\Delta}, C\right)
$$

where $R$ is the Poincare residue operator if $V$ is nonsingular. We show that

THEOREM 5.2. If $V$ has normal crossings, then $\alpha \in H^{p}(W-V)$ can be represented by a differential form with a pole of order one on $V$. 
By the de Rham theorem $H^{p}(W-V ; \mathrm{C})$ can be represented by closed $C^{\infty} p$-forms on $W-V$ modulo exact ones. Furthermore, if $V$ has normal crossings, $\omega$ will be of the type

$$
\omega=\theta \wedge\left(\sum_{U} e_{U} \frac{d f_{U}}{f_{U}}\right)+\eta
$$

where $f_{U}$ is a local defining equation of $V$ in $U$, a suitably chosen coordinate chart in $W$ and $e_{U}$ a partition of unity subordinate to $U$. Also $\eta$ is smooth in $W$ and closed near $V$. Then $R(\omega)=\theta \mid V$, a closed form on $V$, but not necessarily smooth, i.e., $\theta \mid V$ can have a simple pole on the singular locus of $V$.

It is conjectured that for any hypersurface, $\omega$ will have this form, in fact, if $V$ is a subvariety of codimension $q$, then it is conjectured that $\omega=\theta \wedge \lambda+\eta$ where $\lambda$ is a $(q, q-1)$-form with $d \lambda$ the representative of the Poincare dual of $V$ in $W$.

Finally unless otherwise specified, in $\S \S 2-4$, we always have $H_{*}(X)=$ $H_{*}(X ; Z)$, i.e., coefficients in $Z$, and in $\S 5, H_{*}(X)=H_{*}(X ; \mathrm{C})$ and $H^{*}(X)=$ $H^{*}(X ; \mathrm{C})$.

2. Residues of normal crossings. Let $W$ be an $n$-complex dim manifold and $V$ a hypersurface. If $V$ is nonsingular, then we have a map $\tau: H_{p}(V) \rightarrow$ $H_{p+1}(W-V)$ called tubes over cycles which is adjoint to integration over the fibre $R: H^{p+1}(W-V) \rightarrow H^{p}(V)$, i.e. if we represent cohomology of $W-V$ by closed $C^{\infty}$ differential forms on $W-V$ (modulo exact ones), so that if $\omega$ is a closed $(p+1)$-form on $W-V$ and $\alpha \in H_{p}(V)$, then $\int_{\tau(\alpha)} \omega=\int_{\alpha} R(\omega)$. Essentially, if $\gamma_{p}$ is a $p$-cycle which represents $\alpha$, i.e., $\gamma_{p} \in \alpha$, and $s_{\alpha}$ is a local defining equation of $V$ in $U_{\alpha}$ (where we choose coordinates of $W$ in $U_{\alpha}$ so that $s_{\alpha}$ is one of them, which is possible if $V$ is nonsingular), then $\tau\left(\gamma_{p}\right) \cap U_{\alpha}=\left(\left|s_{\alpha}\right|=1\right) \times$ $\left(\gamma_{p} \cap U_{\alpha}\right)$. (Note that $\tau$ is the map which appears in the Thom-Gysin sequence of the normal bundle of $V$ in $W$.) For a precise definition and discussion of $\tau$, see Leray [14], where $\tau$ is called $\delta^{*}=$ coboundary map.

If $V^{\prime}$ is another nonsingular hypersurface of $W$, then we have the maps

$$
H_{p-1}\left(V \cap V^{\prime}\right) \rightarrow H_{p}\left(V-\left(V \cap V^{\prime}\right)\right) \rightarrow H_{p+1}\left(W-\left(V \cup V^{\prime}\right)\right)
$$

and the composition is called reiterated tubes over cycles.

We also have a map on the chain level, $T: C_{p}(V) \rightarrow C_{p+z}(W)$ where if $c_{p}$ is a $p$-chain of $V$ and $s_{\alpha}$ and $U_{\alpha}$ as above, then $T\left(c_{p}\right) \cap U_{\alpha}=\left(\left|s_{\alpha}\right| \leqslant 1\right) \times$ $\left(c_{p} \cap U_{\alpha}\right)$, so that $\partial \circ T=\tau+T \circ \partial$ where $\partial$ is the boundary map (recall $\left.\partial(a \times b)=\partial a \times b+(-1)^{\operatorname{dim} a} a \times \partial b\right)$.

Note that even if $V$ has a nonempty singular locus $S$, if $c_{p}$ is a chain in $V-S$, then we can still speak of the $(p+1)$-chain $\tau\left(c_{p}\right) \subset W-V$ and the $(p+2)$-chain $T\left(c_{p}\right) \subset W-S$. 
2.1 Definition. We say $V$ has normal crossings if $V=\bigcup_{i=1}^{m} V_{i}$ where each $V_{i}$ is a nonsingular hypersurface and if $P \in V_{1} \cap \cdots \cap V_{k}$ and $z_{j}$ is a local defining equation for $V_{j}, 1 \leqslant j \leqslant k$, then $\left(z_{1}, \ldots, z_{k}\right)$ can be extended to a local coordinate system of $W$ about $P$.

Let $i: W-V \rightarrow W$ be the inclusion map and so we get $i_{*}: H_{p}(W-V) \rightarrow$ $H_{p}(W)$ and $\operatorname{Ker} i_{*}=p$-residues of $V=R_{p}(V)$. Also, let $Z_{p}(V, U)$ denote the relative $p$-cycles of $V \bmod U$ for $U \subset V$.

2.2 THEOREM. Let $W$ be an n-dim complex manifold and $V$ a hypersurface with normal crossings. Let $S$ be the singular locus of $V$. Then we can choose $S^{\prime}$ an arbitrary small neighborhood of $S$ in $V$ such that every class of $R_{p}(V)$ has a representative of the form $\tau\left(\gamma_{p-1}\right)$ for some $\gamma_{p-1} \in Z_{p-1}\left(V, S^{\prime}\right)$.

ProOF. Let $\alpha \in R_{p}(V)$ and let $\gamma_{p}$ be a representative of $\alpha$. Then $\gamma_{p}=$ $\partial c_{p+1}, c_{p+1} \subset W$.

Let $M_{i}=i$-fold points of $V$, i.e.,

$$
M_{i}=\underbrace{\bigcup}_{\substack{\left\{j_{1}, \cdots, j_{i}\right\} \\ \text { distinct }}}\left\{V_{j_{1}} \cap \cdots \cap V_{j_{i}}-\bigcup_{\substack{j_{i+1} \neq j_{k} \\ 1<k<i}} V_{j_{1}} \cap \cdots \cap V_{j_{i}} \cap V_{j_{i+1}}\right\}
$$

Therefore, $P \in M_{i}$ implies we can choose coordinates so that $V$ is given by $\Pi_{k=1}^{i} z_{k}=0$ in a neighborhood of $P$ and $\operatorname{dim}_{\mathrm{C}} M_{i}=n-i$ with $M_{i}$ a nonsingular locally closed submanifold and $\partial M_{i}=\bigcup_{j>1} M_{j}$.

Since each $M_{i}$ is a manifold, it makes sense to speak of the transverse intersection of chains with $M_{i}$. Then we let $c_{p+1} \cap M_{i}=\gamma_{i} \in Z_{p+1-2 i}\left(M_{i}\right) \cdot \gamma_{i}$ is a cycle because $\left(\partial c_{p+1}\right) \cap M_{i}=\varnothing=\left(\partial M_{i}\right) \cap \gamma_{i}$. Then we let $i_{0}$ be the largest integer such that $0 \neq\left[\gamma_{i_{0}}\right] \in H_{p+1-2 i_{0}}\left(M_{i_{0}}\right)$, i.e., if $c_{p+1} \cap M_{i}=\varnothing$ for $i>i^{\prime}$ and $\gamma_{i}^{\prime} \sim 0$ in $M_{i^{\prime}}$, then we can deform $c_{p+1}$, keeping $\gamma_{p} \subset W-V$ fixed so that $c_{p+1} \cap M_{i}=\varnothing$. We denote by $i_{0}$ the largest integer such that this process terminates, and $i_{0} \geqslant 1$, since otherwise $\gamma_{p}=\partial c_{p+1}^{\prime}, c_{p+1}^{\prime} \subset W-V$, but then $0=$ $\alpha \in R_{p}(V)$. (Recall $R_{p}(V)$ is a subgroup of $H_{p}(W-V)$ ).)

If $P \in c_{p+1} \cap M_{i_{0}}$ and about $P, V$ is given by $\Pi_{k=1}^{i_{0}} z_{k}=0$, then locally about $P$ we get that $c_{p+1}=C_{1} \times \cdots \times C_{i_{0}} \times \gamma_{i_{0}}$ where $C_{j}=z_{j}$-plane. This is because $c_{p+1}$ hits $P$ transversely and $M_{i_{0}}=\left(0, \ldots, 0, C_{i_{0}+1}, \ldots, C_{n}\right)$.

Since $V$ has normal crossings, if $A$ is a set in $M_{k}$ which misses $\partial M_{k}$, then we can speak of the tubular neighborhood of $A$ in $\bar{M}_{k-1}$, where we put $M_{0}=W-V$. That is, if we have

$$
A \subset V_{1} \cap \cdots \cap V_{k}-\bigcup_{j>k} V_{1} \cap \cdots \cap V_{k} \cap V_{j}
$$


then we can take the tubular neighborhood of $A$ in $V_{1} \cap \cdots \cap \hat{V}_{i} \cap \cdots \cap V_{k}$, since $V_{1} \cap \cdots \cap V_{k}$ is a nonsingular hypersurface in $V_{1} \cap \cdots \cap \hat{V}_{t} \cap \cdots \cap$ $V_{k}$. We denote it by $T_{k, i}(A)=$ solid tube, i.e., all fibres of length $\leqslant$ some $\epsilon$.

Let

$$
T_{k}(A)=\sum_{i=1}^{k} T_{k, i}(A)
$$

Furthermore, by putting appropriate Riemannian metrics in each normal bundle, we can assume that, if $\tau_{k, j}(A)$ denotes the boundary of the tube over $A$, i.e., $T_{k, j}(A)=$ (all vectors $\left.\leqslant \epsilon\right) \times A$ and $\tau_{k, j}(A)=($ all vectors $=\epsilon) \times A$, then we have that as sets $\tau_{k-1, i} \tau_{k, j}(A)=\tau_{k-1, j} \tau_{k, i}(A)$.

However, if $A$ is an oriented chain, we have

$$
\text { 2.3 LeMma. } \tau_{k-1, i} \tau_{k, j}(A)=-\tau_{k-1, j} \tau_{k, i}(A) \text {. }
$$

Proof. See Leray $[14$, p. 132] for proof. Essentially this is true because in a fibre bundle, if $c_{1}$ and $c_{2}$ are oriented cycles, then $c_{1} \times c_{2}=-c_{2} \times c_{1}$. This completes the proof of the lemma.

Let

$$
\tau_{k}(A)=\sum_{i=1}^{k} \tau_{k, i}(A)
$$

2.4 Corollary. $\tau_{k-1} \tau_{k}(A)=0$, if $A$ is an oriented chain.

Proof. $\tau_{k-1} \tau_{k}(A)$ is a sum of pairs of the form $\tau_{k-1, i} \tau_{k, j}(A)+$ $\tau_{k-1, j} \tau_{k, i}(A)$ which is zero by the lemma. Q.E.D. for Corollary 2.4.

If $A \subset V_{1} \cap \cdots \cap V_{k}$ let

$$
\tilde{T}_{i} \cdots \widetilde{T}_{k}(A)=\sum_{i_{1}<\cdots<i_{k-i+1}} T_{i, i_{1}} T_{i+1, i_{2}<k} \cdots T_{k, i_{k-i+1}}(A) .
$$

Note that $\widetilde{T}_{k}(A)=T_{k}(A)$.

Let $\ddot{\gamma}_{i_{0}}=\gamma_{i_{0}}$ and by induction define for $i<i_{0}$

$$
\tilde{\gamma}_{i}=\gamma_{i}-\left[\widetilde{T}_{i+1} \tilde{\gamma}_{i+1}+\widetilde{T}_{i+1} \widetilde{T}_{i+2} \tilde{\gamma}_{i+2}+\cdots+\widetilde{T}_{i+1} \cdots \widetilde{T}_{i_{0}} \tilde{\gamma}_{i_{0}}\right]
$$

$\underset{\widetilde{T}}{\text { after deforming }} C_{p+1}$ (leaving $\gamma_{p}$ fixed) such that $\widetilde{T}_{i+1} \tilde{\gamma}_{i+1}+\cdots+\widetilde{T}_{i+1} \cdots$ $\widetilde{T}_{i_{0}} \widetilde{\gamma}_{i_{0}}$ is a $(p+1-2 i)$-dim chain contained in $\gamma_{i}$, which is possible by transversality.

2.5 Lemma. $\partial \tilde{\gamma_{i}}=-\tau_{i+1} \tilde{\gamma_{i+1}}$ for $i<i_{0}$. 
PROOF. For $i=i_{0}-1$,

$$
\partial \tilde{\gamma}_{i_{0}-1}=\partial \gamma_{i_{0}-1}-T_{i_{0}-1} \partial \gamma_{i_{0}}-\tau_{i_{0}} \gamma_{i_{0}}=-\tau_{i_{0}} \gamma_{i_{0}}
$$

since the $\gamma_{j}$ are cycles.

For $i<i_{0}-1$, since $\tau_{i, j} T_{i+1, k}=T_{i, k} \tau_{i+1, j}$ (because the $T_{i, j}$ are locally products of 2-dim chains), we have that

$$
\begin{gathered}
\sum_{\substack{i_{1}<\cdots<i_{k} \\
i<i_{j}<m}} \sum_{j=1}^{k} T_{i_{, i} i_{1}} \cdots T_{i+j-1, i_{j}} \tau_{i+j, i_{j+1}} T_{i+j+1, i_{j+2}} \cdots T_{i+k-1, i_{k}}(A) \\
\quad \sum_{\substack{i_{1}<\cdots<i_{k-1} \\
i<i_{j}<m}} T_{i, i_{1}} \cdots T_{i+k-2, i_{k-1}} \tau_{i+k-1}(A)
\end{gathered}
$$

where $\tau_{i+k+1}$ is defined in (2). This is a straightforward combinatorial argument.

Hence by induction on $i$ and the above equality, we get

$$
\begin{aligned}
\partial\left(\tilde{T}_{i+1} \cdots \tilde{T}_{i+j} \tilde{\gamma}_{i+j}\right)= & \tilde{T}_{i+1} \cdots \tilde{T}_{i+j-1} \tau_{i+j} \tilde{\gamma}_{i+j} \\
& -\tilde{T}_{i+1} \cdots \tilde{T}_{i+j} \tau_{i+j+1} \tilde{\gamma}_{i+j+1}
\end{aligned}
$$

Then a straightforward computation and the induction hypothesis yield $\partial \tilde{\gamma}_{i}=$ $-\tau_{i+1} \tilde{\gamma}_{i+1}$. Q.E.D. for Lemma 2.5 .

Let $T^{\prime}=T_{1} \tilde{\gamma}_{1}$ which is $(p+1)$-dim chain in $W$; deform $c_{p+1}$ (leaving $\gamma_{p}$ fixed) so that $T^{\prime} \subset c_{p+1}$. Then $\partial\left(c_{p+1}-T^{\prime}\right)=\gamma_{p}-\tau_{1} \tilde{\gamma}_{1}-T_{1}\left(\partial \tilde{\gamma}_{1}\right)$. Now, $-T_{1} \partial \tilde{\gamma}_{1}=T_{1} \tau_{2} \tilde{\gamma}_{2}$ by Lemma 2.5 , and $\partial\left(T_{1}\left(\partial \tilde{\gamma}_{1}\right)\right)=\tau_{1} \tau_{2} \tilde{\gamma}_{2}=0$ by Corollary 2.4; therefore $T_{1}\left(\partial \tilde{\gamma}_{1}\right)$ is an absolute cycle which implies $\tau_{1}\left(\tilde{\gamma}_{1}\right)$ is also an absolute cycle.

2.6 LEMMA. $T_{1}\left(\partial \tilde{\gamma}_{1}\right)=-T_{1} \tau_{2} \tilde{\gamma}_{2}=\partial T^{\prime \prime}$ where

$$
T^{\prime \prime}=\tilde{T}_{1} \tilde{T}_{2} \tilde{\gamma}_{2}+\tilde{T}_{1} \widetilde{T}_{2} \tilde{T}_{3} \tilde{\gamma}_{3}+\cdots+\widetilde{T}_{1} \cdots \widetilde{T}_{i_{0}} \tilde{\gamma}_{i_{0}}
$$

ProOF. We have

$$
\tilde{T}_{1} \cdots \tilde{T}_{k} \tilde{\gamma}_{k}=\tilde{T}_{1} \cdots \tilde{T}_{k-1} \tau_{k} \tilde{\gamma}_{k}-\tilde{T}_{1} \cdots \tilde{T}_{k} \tau_{k+1} \tilde{\gamma}_{k+1}
$$

by Lemma 2.5 . Then computing $T^{\prime \prime}$ with the above equality yields a telescoping series whose first term is $-T_{1} \tau_{2} \widetilde{\gamma}_{2}$ and whose last term is $\widetilde{T}_{1} \cdots \widetilde{T}_{i_{0}} \partial \widetilde{\gamma}_{i_{0}}$ $=0$. Q.E.D. for Lemma 2.6.

Therefore, let $T=T^{\prime}+T^{\prime \prime} \subset c_{p+1}$ and $c_{p+1} \cap V \subset T$. Then we have 
$c_{p+1}-T \subset W-V$ and

$$
\partial\left(c_{p+1}-T\right)=\gamma_{p}-\tau_{1}\left(\tilde{\gamma}_{p-1}\right)+T_{1}\left(\partial \tilde{\gamma}_{1}\right)-T_{1}\left(\partial \tilde{\gamma}_{1}\right)=\gamma_{p}-\tau_{1}\left(\tilde{\gamma}_{p-1}\right)
$$

Since we have that $\tilde{\gamma}_{p-1,1} \in Z_{p-1}\left(V, S^{\prime}\right)$ where $S^{\prime}$ is an arbitrary small neighborhood in $V$ of $U_{j>2} M_{j}=S=$ singular locus of $V$ by choosing the tubular neighborhoods sufficiently small. Q.E.D. for Theorem 2.2.

The $\tau$ we have constructed is not well defined on homology, i.e., $\gamma_{p-1}$ can bound in $V$ but $\tau_{1}\left(\tilde{\gamma}_{1}\right)$ might not bound in $W-V$. However, if $\gamma_{p-1}$ does bound in $V$, then it is a tube over a lower-dimensional cycle in $V$. Before proving this we will need some more notation.

Again, let $V=\bigcup_{i=1}^{m} V_{i}$ have normal crossings. Let $M_{i}$ be the $i$-tuple points, i.e.,

$$
M_{i}=\bigcup_{\text {distinct } i \text {-tuples }} M\left(j_{1}, \ldots, j_{i}\right)
$$

where

$$
M\left(j_{1}, \ldots, j_{i}\right)=V_{j_{1}} \cap \cdots \cap V_{j_{i}}-\bigcup_{k \neq j_{l} ; 1 \leqslant l \leqslant i} V_{k} \cap V_{j_{1}} \cap \cdots \cap V_{i_{i}} .
$$

Let $\bar{M}\left(j_{1}, \ldots, j_{i}\right)=$ closure of $M\left(j_{1}, \ldots, j_{i}\right)=V_{j_{1}} \cap \cdots \cap V_{j_{i}}$. For notational completeness, let $M_{0}=W-V$. Also, $\bar{M}_{i}=\bigcup^{1} \bar{M}\left(j_{1}, \ldots, j_{i}\right)$.

At each component of $M_{i+j}$ there are $\left(\begin{array}{c}i+j \\ i\end{array}\right)=\left(\begin{array}{c}i+j \\ j\end{array}\right)$-components of $M_{i}$ nearby, i.e., if locally $M_{i+j}=\left\{z_{1} \cdots z_{i+j}=0\right\}$, then $\left\{z_{k_{1}} \cdots z_{k_{i}}=0\right\}$ for all distinct subsets $\left\{k_{1}, \ldots, k_{i}\right\} \subset\{1, \ldots, i+j\}$ defines the $\left(\begin{array}{c}i+j \\ i\end{array}\right)$-components. Now $\left(\begin{array}{c}i+j \\ i\end{array}\right) \geqslant j+1$ and we say that:

2.7 Definition. $(j+1)$ of the components, say $\left\{k_{1 s}, \ldots, k_{i s}\right\}, s=$ $1, \ldots, j+1$, are tubular if $\bigcap_{s=1}^{j+1}\left\{k_{1 s}, \ldots, k_{i s}\right\}=\left\{h_{1}, \ldots, h_{i-1}\right\}$, i.e., $\bigcap_{s=1}^{j+1} M\left(k_{1 s}, \ldots, k_{i s}\right)$ is a component of $\bar{M}_{i-1}$.

Note that $\left|\bigcap_{s=1}^{j+1}\left\{k_{1 s}, \ldots, k_{i s}\right\}\right| \leqslant i-1$ where the $k_{i s}$ are arbitrary. Another way to see this is: if say $M(1, \ldots, i+j) \subset M(i+j)$ and take any $M_{i-1}$ nearby, say $M(1, \ldots, i-1)$. Then there are exactly $(j+1)$-components of $M_{i}$ between $M(1, \ldots, i-1)$ and $M(1, \ldots, i+j)$, namely $M(1, \ldots, i-1, k), k=i, \ldots, i+j$, and these $M(1, \ldots, i-1, k)$ are tubular.

We let $H_{p}\left(\bar{M}_{i}\right)_{\Delta}$ (the tubular cycles) be those classes of $H_{p}\left(\bar{M}_{i}\right)$ which have a representative such that each component $\gamma_{p}$ has the following properties:

(a) If $\gamma_{p} \cap M\left(k_{1}, \ldots, k_{i+j}\right) \neq \varnothing$, then $\operatorname{dim}_{R} M\left(k_{1}, \ldots, k_{i+j}\right) \cap \gamma_{p}=$ $p-2 j$, i.e., they intersect transversely.

(b) Of the $\left(\begin{array}{c}j+1 \\ i\end{array}\right)$-components of $M_{i}$ at $M\left(k_{1}, \ldots, k_{i+j}\right), \gamma_{p}$ meets exactly 
$(j+1)$ of them in a nonempty transverse intersection.

(c) These $(j+1)$-components form a tubular set.

This means, for example, if $\operatorname{dim}_{\mathrm{C}} W=2$ and $V=V_{1} \cup V_{2}$ with $V_{i}$ being nonsingular curves which intersect in double points, then if $\gamma_{1}$ is a line in $V_{1}$ from one double point to a different one and a line in $V_{2}$ connecting the same double points, then (a) implies that $\left[\gamma_{1}\right] \notin H_{1}\left(\bar{M}_{1}\right)_{\Lambda}$. (b) implies $\left[V_{1}\right] \notin H_{2}\left(\bar{M}_{1}\right)_{\Delta}$. Also note that in Theorem $2.2 H_{p-1}\left(\bar{M}_{1}\right)_{\Delta}$ is precisely those classes for which we constructed tubes in $W-V$. We did not have to worry about tubular then, since for $i=1,\left(\begin{array}{c}1+j \\ 1\end{array}\right)=j+1$ and they are always tubular.

Let $\tau_{i}: H_{p}\left(\bar{M}_{i}\right)_{\Delta} \rightarrow H_{p+1}\left(M_{i-1}\right)$ be the map defined in Theorem 2.2. There we had it for $i=1$, but if one checks one easily sees that tubular is precisely what one needs for $i>1$. In fact, if $M\left(k_{1 s}, \ldots, k_{i s}\right), s=1, \ldots, j+1$, are the tubular components, then they are tubular to some $M_{i-1}$, say $M\left(h_{1}, \ldots, h_{i-1}\right)$. Then $M\left(h_{1}, \ldots, h_{i-1}\right)$ plays the role of $M_{0}=W-V$ in Theorem 2.2. Let $\tilde{\tau}_{i}: H_{p}\left(M_{i}\right) \rightarrow H_{p+1}\left(M_{i-1}\right)$ be the map which puts a tube over a cycle in just one normal direction, i.e., $\tilde{\tau}_{i}=\tau_{i, j}$ for some $j$.

2.8 Corollary. $R_{p}(V)$ is generated by $\tau_{1} H_{p-1}\left(\bar{M}_{1}\right)_{\Delta}, \tilde{\tau}_{1} \tau_{2} H_{p-2}\left(\bar{M}_{2}\right)_{\Delta}$, $\ldots, \tilde{\tau}_{1} \cdots \tilde{\tau}_{p-1} \tau_{p} H_{0}\left(\bar{M}_{p}\right)_{\Delta}$.

2.9 Definition. $\tilde{\tau}_{1} \cdots \tilde{\tau}_{q-1} \tau_{q} H_{p-q}\left(\bar{M}_{q}\right)_{\Delta}$ are called the geometric $(p-q)$-residues of $R_{p}(V)$.

Note. The notion of geometric $q$-residue follows Hodge-Atiyah [11], whose definition follows Zariski $\left[28\right.$, p. 150]. Also $\tilde{\tau}_{i}=\tau_{i, j}$ does not depend on which $j$ we choose because $\tilde{\tau}_{1} \cdots \tilde{\tau}_{p-q-1} \tau_{p-q}\left(\gamma_{p-q}\right)$ will only differ by a sign if we choose the $j$ 's in different order. This corollary is true for all $p \leqslant 2 n$, since the only difficulty would be for $p=2 n$, but $R_{2 n}(V)=0$.

PROOF. We prove this by induction on $m=$ number of components of $V$. For $m=1$, this is clear. Therefore assume done for less than $m$.

Let $\alpha \in R_{p}(V)$ and $\gamma_{p}$ be a representative of $\alpha$ so that $\gamma_{p}=\partial c_{p+1}$, $c_{p+1} \cap V=\gamma_{p-1},\left[\gamma_{p-1}\right] \in H_{p-1}(V)_{\Delta}$ and $\gamma_{p} \sim \tau\left(\gamma_{p-1}\right)$ by Theorem 2.2.

If $\gamma_{p-1} \neq 0$ in $V$, then we have $\gamma_{p} \in \tau_{1} H_{p-1}\left(\bar{M}_{1}\right)_{\Delta}$. Suppose $\gamma_{p-1}=\partial c_{p}$ for $c_{p} \subset V$. Then choose the largest $i_{0}$ such that $\gamma_{p-1} \cap M_{i_{0}} \neq \varnothing$ and $\operatorname{dim} \gamma_{p-1}$ $\cap M_{i_{0}}=p+1-2 i_{0}$.

If $i_{0}=1$, then $\gamma_{p-1} \subset M_{1}$ and we can apply the induction hypothesis; namely, let $W^{\prime}=V_{i}, V^{\prime}=S \cap V_{i}$ where $S=$ singular locus of $V=\bar{M}_{2}$ and $\gamma_{p-1} \cap V_{i}=\gamma_{p-1, i} \in H_{p-1}\left(W^{\prime}-V^{\prime}\right)$. Then since $V^{\prime}$ has $(m-1)$-components, we get $\gamma_{p-1, i}$ is generated by $\tau_{1}^{\prime} H_{p-2}\left(\bar{M}_{1}^{\prime}\right)_{\Delta}, \ldots, \tau_{1}^{\prime} \ldots$ $\tilde{\tau}_{p-2}^{\prime} \tau_{p-1}^{\prime} H_{0}\left(\bar{M}_{p-1}^{\prime}\right)_{\Delta}$. But $\bar{M}_{1}^{\prime} \subset \bar{M}_{i+1}$ and $\tau_{i}^{\prime}=\tau_{i+1}, \tilde{\tau}_{1}^{\prime}=\tilde{\tau}_{i+1}$ so that we 
have $\gamma_{p-1, i}$ is generated by $\tau_{2} H_{p-2}\left(\bar{M}_{2}\right)_{\Delta}, \ldots, \tilde{\tau}_{2} \cdots \tilde{\tau}_{p-1} \tau_{p} H_{0}\left(\bar{M}_{p}\right)_{\Delta}$ which implies $\tau_{1}\left(\gamma_{p-1}\right)=\Sigma_{i} \tau_{1, i}\left(\gamma_{p-1, i}\right)$ is generated by $\tilde{\tau}_{1} \tau_{2} H_{p-2}\left(\bar{M}_{2}\right)_{\Delta}, \ldots, \tilde{\tau}_{1} \ldots$ $\tilde{\tau}_{p-1} \tau_{p} H_{0}\left(M_{p}\right)_{\Delta}$.

Assume $i_{0}>1$. Let $\gamma_{i_{0}}=\gamma_{p-1} \cap M_{i_{0}} \in H_{p+2-2 i_{0}}\left(M_{i_{0}}\right)$. Now, $\operatorname{dim}_{R} c_{p} \cap \bar{M}_{i_{0}} \leqslant p+2-2 i_{0}$. Suppose $\operatorname{dim}_{R} c_{p} \cap \bar{M}_{i_{0}}=p+2-2 i_{0}$ and let $c_{p} \cap \bar{M}_{i_{0}}=c_{i_{0}}$. If $c_{i_{0}} \subset M_{i_{0}}$, then we can construct the tube over $c_{p}$ such that $\tau\left(c_{p}\right) \subset W-V$ and $\partial \tau\left(c_{p}\right)=-\tau\left(\gamma_{p-1}\right)=-\gamma_{p}$, i.e., $\gamma_{p} \sim 0$ in $W-V$. The construction is as follows: Let $c_{p} \cap M_{i}=c_{i}, 1 \leqslant i \leqslant i_{0}$. Then put $\tilde{c}_{i_{0}}=c_{i_{0}}$ and by induction define for $j<i_{0}$

$$
\tilde{c}_{j}=c_{j}-\left[\tilde{T}_{j+1} \tilde{c}_{j+1}+\cdots+\tilde{T}_{j+1} \cdots \tilde{T}_{i_{0}} \tilde{c}_{i_{0}}\right]
$$

after deforming as in Theorem 2.2. This is possible because of tubularness.

But $\partial \tilde{c}_{j}=-\tau_{j+1} \tilde{c}_{j+1}, j<i_{0}$, and $\partial \tilde{c}_{1}=\tilde{\gamma}_{1}$. So we form $\tau_{1}\left(\tilde{c}_{1}\right) \subset W-V$ and $\partial \tau_{1} \widetilde{c}_{1}=-\tau_{1}\left(\partial \widetilde{c}_{1}\right)=-\tau_{1} \tilde{\gamma}_{1}=-\gamma_{p}$.

Next, suppose $c_{p} \cap M_{i_{0}+1} \neq \varnothing$ which implies $\operatorname{dim}_{R} c_{p} \cap M_{i_{0}+1}=p-2 i_{0}$. However, we cannot, in general, construct the tube over $c_{p}$ in $W-V$ because we might have $M_{i_{0}+1}$ contained in some component of $\bar{M}_{i_{0}}$ with $c_{p}$ missing this component, e.g., let $p=4, i_{0}=2$ and locally we have coordinates $(x, y, z)$ with $V=\{x y z=0\}$.

Let

$$
\gamma_{3}=\left[\{x=0\} \times C_{y} \times(|z|=1)\right] \cup\left[C_{x} \times\{y=0\} \times(|z|=1)\right] .
$$

Then

$$
c_{4}=\left[\{x=0\} \times \mathbf{C}_{y} \times(|z| \leqslant 1)\right] \cup\left[\mathbf{C}_{x} \times\{y=0\} \times(|z| \leqslant 1)\right]
$$

and we connot construct $\tau\left(c_{4}\right) \subset W-V$ because at $(0,0,0) \in M_{3}, \operatorname{dim} c_{4} \cdot M_{3}$ $=0$ but $M_{3} \subset \bar{M}(y, z)$ and $c_{4} \cdot M(y, z)=\varnothing$. We notice, however, that

$$
\begin{array}{r}
\tau\left(\gamma_{3}\right)=\left[(|x|=1) \times \mathbf{C}_{y}-(|y| \leqslant 1) \times(|z|=1)\right] \\
\cup\left[\mathbf{C}_{x}-|x| \leqslant 1 \times(|y|=1) \times(|z|=1)\right] .
\end{array}
$$

So if instead of coming down to $V$ so that we get $\gamma_{3}$, we first come down to $V$ via $|z|=1$, we get

$$
\begin{aligned}
& \gamma_{3}^{\prime}= {\left[(|x|=1) \times \mathbf{C}_{y}-(|y| \leqslant 1) \times\{z=0\}\right] } \\
& \cup\left[\mathbf{C}_{x}-(|x| \leqslant 1) \times(|y|=1) \times\{z=0\}\right]
\end{aligned}
$$

and $\gamma_{3}^{\prime}=\tau\left(\gamma_{2}^{\prime}\right), \gamma_{2}^{\prime} \in H_{2}\left(\bar{M}_{2}\right)_{\Delta}$ where 


$$
\gamma_{2}^{\prime}=\left[\{x=0\} \times C_{y} \times\{z=0\}\right] \cup\left[C_{x} \times\{y=0\} \times\{z=0\}\right] .
$$

We can also do this in the general case: $\gamma_{i_{0}} \in H_{p+1-2 i_{0}}\left(M_{i_{0}}\right)$ and assume $\boldsymbol{\gamma}_{i_{0}}$ is connected (if not, do each component one at a time). This implies $\boldsymbol{\gamma}_{\boldsymbol{i}_{0}} \subset$ $M\left(1, \ldots, i_{0}\right)$. Then if $W^{\prime}=\bar{M}\left(1, \ldots, i_{0}\right)$ and $V^{\prime}=\bigcup_{j>i_{0}} \bar{M}\left(1, \ldots, i_{0}, j\right)$ then $\gamma_{i_{0}}$ is a residue of $V^{\prime}$ in $W^{\prime}$ and $W^{\prime}$ has less than $m$ components which implies by induction that we have

$$
\gamma_{i_{0}}=\tilde{\tau}_{i_{0}+1} \cdots \tilde{\tau}_{i_{0}+q-1} \tau_{i_{0}+q}\left(\gamma_{i_{0}+q}\right), \quad \gamma_{i_{0}+q} \in H_{p+1-2 i_{0}-q}\left(\bar{M}_{i_{0}+q}\right)_{\Delta} .
$$

Thus locally we have for $P \in \gamma_{i_{0}}$ and $U$ a coordinate chart about $P$ with local coordinates $\left(z_{1}, \ldots, z_{n}\right)$ and $V=\left\{z_{1} \cdots z_{i_{0}}=0\right\}$ that $\gamma_{p-1} \cap U=c_{2 i_{0}-2} \times\left(\left|z_{i_{0}+1}\right|-1\right) \times \cdots \times\left(\left|z_{q-1}\right|=1\right) \times\left\{\tau_{q}\left(\gamma_{i_{0}+q}\right) \cap U\right\}$ where

$$
\begin{aligned}
c_{2 i_{0}-2}=U \cap & {\left[\left\{z_{1}=0\right\} \times \mathbf{C}_{z_{2}} \times \cdots \times \mathbf{C}_{z_{i_{0}}} \cup \mathbf{C}_{z_{1}} \times\left\{z_{2}=0\right\}\right.} \\
& \left.\times \mathbf{C}_{z_{3}} \times \cdots \times \mathbf{C}_{z_{i_{0}}} \cup \cdots \cup \mathbf{C}_{z_{1}} \times \cdots \times \mathbf{C}_{z_{i_{0}-1}} \times\left\{z_{i_{0}}=0\right\}\right] .
\end{aligned}
$$

Claim. $\gamma_{p} \in \tilde{\tau}_{1} \cdots \tilde{\tau}_{q} \tau_{q+1} H_{p-q-1}\left(\bar{M}_{q+1}\right)_{\Delta}$.

We can assume $q=1$, else rather than dropping down to $V$ by the tube which yields $\gamma_{p-1}$, we drop down via $\tilde{\tau}_{i_{0}+1}$ and this yields a $\gamma_{p-1}^{\prime} \subset M_{1}$ and we can proceed by the induction hypothesis.

Then $\gamma_{i_{0}}=\tau_{i_{0}+1}\left(\gamma_{p-1, i_{0}+1}\right), \gamma_{i_{0}+1} \in H_{p-2 i_{0}}\left(\bar{M}_{i_{0}+1}\right)_{\Delta}$. Thus

$$
\tau_{1}\left(c_{2 i_{0}-2} \times \tau_{i_{0}+1}\left(\gamma_{i_{0}+1}\right)\right) \sim \tilde{\tau}_{1} \tau_{2}\left(c_{2 i_{0}-2} \times \gamma_{i_{0}+1}\right) \in \tilde{\tau}_{1} \tau_{2} H_{p-2}\left(\bar{M}_{2}\right)_{\Delta}
$$

To see this, consider the following example: $p=6, i_{0}=2$ and locally

$$
\begin{aligned}
\gamma_{p-1}= & {\left[\mathbf{C}_{z_{1}} \times\left\{z_{2}=0\right\} \cup\left\{z_{1}=0\right\} \times C_{z_{2}}\right] } \\
& \times\left[C_{z_{3}}-\left(\left|z_{3}\right| \leqslant 1\right) \times\left(\left|z_{4}\right|=1\right) \cup\left(\left|z_{3}\right|=1\right) \times C_{z_{4}}-\left(\left|z_{4}\right| \leqslant 1\right)\right]
\end{aligned}
$$

and

$$
\left\{C_{z_{3}}-\left(\left|z_{3}\right| \leqslant 1\right) \times\left|z_{4}\right|=1\right\} \cup\left\{\left|z_{3}\right|=1 \times C_{z_{4}}-\left(\left|z_{4}\right| \leqslant 1\right\}=\tau_{2}\left(\gamma_{3}\right)\right.
$$

where 


$$
\begin{aligned}
\gamma_{3}= & \left\{z_{1}=0\right\} \times\left\{z_{2}=0\right\} \times C_{z_{3}} \times\left\{z_{4}=0\right\} \\
& \cup\left\{z_{1}=0\right\} \times\left\{z_{2}=0\right\} \times\left\{z_{3}=0\right\} \times C_{z_{4}} .
\end{aligned}
$$

So before we constructed tubes, we were considering

$$
\begin{aligned}
\mathbf{C}_{z_{1}} \times\left\{z_{2}=\right. & 0\} \times \mathbf{C}_{z_{3}} \times\left\{z_{4}=0\right\} \cup \mathbf{C}_{z_{1}} \times\left\{z_{2}=0\right\} \\
& \times\left\{z_{3}=0\right\} \times \mathbf{C}_{z_{4}} \cup\left\{z_{1}=0\right\} \times \mathbf{C}_{z_{2}} \times \mathbf{C}_{z_{3}} \\
& \times\left\{z_{4}=0\right\} \cup\left\{z_{1}=0\right\} \times \mathbf{C}_{z_{2}} \times\left\{z_{3}=0\right\} \times\left\{z_{4}=0\right\} \\
= & \bar{M}(1,3) \cup \bar{M}(1,4) \cup \bar{M}(2,3) \cup \bar{M}(2,4) .
\end{aligned}
$$

But $\bar{M}(1,3)+\bar{M}(2,3) \sim \bar{M}(3,4)$ because $\partial \bar{M}(3)=\bar{M}(1,3)+\bar{M}(2,3)+$ $\bar{M}(3,4)$. Therefore, what we want to construct the tube over is really $\bar{M}(1,4)+$ $\bar{M}(2,4)+\bar{M}(3,4) \in H_{4}\left(\bar{M}_{2}\right)_{\Delta}$, i.e., $\gamma_{p}=\tau_{1} \tau_{2}\left(\gamma_{2}\right), \gamma_{2} \in H_{p-1}\left(\bar{M}_{2}\right)_{\Delta}$. Thus what we are saying in this example is that if we set

$$
\begin{array}{ll}
c_{1}=\mathrm{C}_{z_{1}}-\left(\left|z_{1}\right| \leqslant 1\right) \times\left(\left|z_{2}\right|=1\right), & c_{2}=\left(\left|z_{1}\right|=1\right) \times \mathbf{C}_{z_{2}}-\left(\left|z_{2}\right| \leqslant 1\right), \\
c_{3}=\mathbf{C}_{z_{3}}-\left(\left|z_{3}\right| \leqslant 1\right) \times\left(\left|z_{4}\right|=1\right), & c_{4}=\left(\left|z_{3}\right|=1\right) \times \mathbf{C}_{z_{4}}-\left(\left|z_{4}\right| \leqslant 1\right)
\end{array}
$$

and

$$
c_{5}=\mathrm{C}_{z_{1}}-\left(\left|z_{1}\right| \leqslant 1\right) \times \mathrm{C}_{z_{2}}-\left(\left|z_{2}\right| \leqslant 1\right) \times\left(\left|z_{3}\right|=1\right) \times\left(\left|z_{4}\right|=1\right),
$$

then we have that $\gamma_{p}=c_{1} \times c_{3}+c_{2} \times c_{3}+c_{1} \times c_{4}+c_{2} \times c_{4}$ is homologous in $W-V$ to $\gamma_{p}^{\prime}=c_{1} \times c_{3}+c_{2} \times c_{3}+c_{5}$ with $c_{5} \sim c_{1} \times c_{4}+c_{2} \times c_{4}$ corresponding to $\bar{M}(1,3)+\bar{M}(2,3) \sim \bar{M}(3,4)$.

The general case, locally, is the same as the above example, i.e., given $U$ open with $V$ given in $U$ by $\Pi_{i=1}^{k} z_{i}=0$, then we can transform $\gamma_{p-1}$ in $U$ so that

$\gamma_{p-1}=\sum_{j=1}^{i_{0}} C_{1} \times \cdots \times C_{j-1} \times\{0\} \times C_{j+1} \times \cdots \times C_{i_{0}} \times \tau_{i_{0}+1}\left(\gamma_{i_{0}+1}\right)$

Then

$$
\begin{aligned}
\gamma_{i_{0}+1}=\sum_{j=1}^{l} & \mathbf{C}_{i_{0}+1} \times \cdots \times \mathrm{C}_{i_{0}+j-1} \times\{0\} \\
& \times \mathbf{C}_{i_{0}+j+1} \times \cdots \times \mathrm{C}_{i_{0}+l} \times \gamma_{i_{0}+m+1},
\end{aligned}
$$

where 


$$
\gamma_{i_{0}+m+1}= \begin{cases}P_{i_{0}+m+1} \times \cdots \times P_{n} & \text { if } p-2 i_{0}=2 l, \\ \gamma_{1} \times P_{i_{0}+m+2} \times \cdots \times P_{n} & \text { if } p-2 i_{0}=2 l+1,\end{cases}
$$

and $P_{i} \in C_{i}-\{0\}, \gamma_{1}$ is a line in $C_{i_{0}+m+1}-\{0\}$. (Recall, $\operatorname{dim}_{R} \gamma_{i_{0}+1}=p-$ $2 i_{0}$.) We let $m=\min \left(k, i_{0}+1\right)$ and $\pi_{m}: \mathbf{C}^{n} \rightarrow \mathbf{C}^{m}$ be the projection on the first $m$-coordinates. Then, locally, before we construct $\tau_{i_{0}+1}$, we have the $(p-2)$-dim chain

$$
\gamma_{p-2}=\sum_{i=1}^{i_{0}} \sum_{j=i_{0}+1}^{m} \pi_{m}(M(i, j)) \times \gamma
$$

where $\gamma$ is a $(p-2 m)$-dim chain in $0 \times \cdots \times 0 \times \mathbf{C}_{m+1} \times \cdots \times \mathbf{C}_{n}$ and $M(i, j)$ means $\left\{z_{i}=0\right\} \cap\left\{z_{j}=0\right\}$. By keeping $\gamma$ fixed, we can work in $\pi_{m}\left(\mathbf{C}^{n}\right)$ $=\pi_{m}(U)$ and we have

2.10 LEMMA. $\sum_{i=1}^{i_{0}} \sum_{j=i_{0}+1}^{m} \pi_{m}(M(i, j)) \sim \Sigma_{i<m} \pi_{m}(M(i, m))$ in $\pi_{m}(U)$.

Proof. To fix some consistent orientation in $M(i)$, we say $M(i, j)=M(j, i)$ and

$$
\partial M(i)=\sum_{j \neq i} \epsilon(j, i) M(j, i) \quad \text { where } \epsilon(j, i)= \begin{cases}+1, & j<i \\ -1, & j>i\end{cases}
$$

Then

$$
\partial \pi_{m}(M(i))=\sum_{j<m ; j \neq i} \epsilon(j, i) \pi_{m}(M(j, i))
$$

Hence

$$
\sum_{i<i_{0}+k} \pi_{m}\left(M\left(i, i_{0}+k\right)\right) \sim \sum_{i>i_{0}+k} \pi_{k}\left(M\left(i, i_{0}+k\right)\right) \text { in } \pi_{m}(U) \text { for all } k .
$$

Thus, for $k=1$,

$$
\sum_{1=1}^{i_{0}} \sum_{j=i_{0}+1}^{m} \pi_{m}(M(i, j)) \sim \sum_{i=1}^{i_{0}} \sum_{j=i_{0}+2}^{m} \pi_{m}(M(i, j))+\sum_{i>i_{0}+1} \pi_{m}\left(M\left(i, i_{0}+1\right)\right)
$$

for $k=2$,

$$
\sim \sum_{i=1}^{i_{0}} \sum_{j=i_{0}+3}^{m} \pi_{m}(M(i, j))+\sum_{i>i_{0}+2} \pi_{m}\left(M\left(i, i_{0}+1\right)\right)+\pi_{m}\left(M\left(i, i_{0}+2\right)\right)
$$

for $k=m-1$, 


$$
\sim \sum_{i=1}^{i_{0}} \pi_{m} M(i, m)+\sum_{k=1}^{m-i_{0}-1} \pi_{m} M\left(m, i_{0}+m\right)=\sum_{i<m} \pi_{m}(M(i, m))
$$

Q.E.D. for Lemma 2.10 .

Note. This lemma is true for any $m$ chosen, $1 \leqslant m \leqslant n=\operatorname{dim}_{\mathbf{C}} W$.

Then let $\gamma_{p-2}^{\prime}=\Sigma_{i<m} \pi_{m}(M(i, m)) \times \gamma$ and $\gamma_{p-2}^{\prime} \sim \gamma_{p-2}$ in $U$ $(\bmod \partial U)$ and $\tau_{2}\left(\gamma_{p-2}^{\prime}\right) \subset M_{1}$ by choice of $m$ with $\tau_{2}\left(\gamma_{p-2}^{\prime}\right) \sim \gamma_{p-1}$ $(\bmod \partial U)$.

Thus, locally there is no problem. The difficulty is global: Can we make these local changes in a consistent manner, since we change $\gamma_{p-2}$ at $\partial U$.

To do the global case, we will construct a polygon $\Gamma$ of $\operatorname{dim}_{R}=n_{0}-1$ where

$$
n_{0}=\max _{i}\left\{\exists \text { local coordinates so that } \prod_{j=1}^{i} z_{j}=0 \text { defines } V\right\}
$$

i.e., $n_{0}=$ maximum number of components of $V$ which intersect at a point. Then $n_{0} \leqslant \min (n, m), n=\operatorname{dim}_{\mathrm{C}} W, m=$ number of components of $V$. In general, $n_{0}=n$.

Before constructing $\Gamma$, we note that we can assume the $M\left(i_{1}, \ldots, i_{k}\right)=$ $V_{i_{1}} \cap \cdots \cap V_{i_{k}}$ are connected for all $\left\{i_{1}, \ldots, i_{k}\right\} \subset\{1, \ldots, m\}$. This is because, if not, then perform monoidal transforms with centers at all but one of the components of $M\left(i_{1}, \ldots, i_{k}\right)$ and this will create new $V_{i}, i>m$, but with only one component for $M\left(i_{1}, \ldots, i_{k}\right)$. Then Proposition 3.1 shows that $R_{p}(V)$ is invariant under such transformations.

Note. The connectedness of the $M\left(i_{1}, \ldots, i_{k}\right)$ simplifies the geometric arguments which follow but is not necessary.

Now, to construct $\Gamma$ : to each $M(i)=V_{i} \subset V$, associate an $\left(n_{0}-1\right)$-dim face, call it $S_{i}$. If $\varnothing \neq M\left(i_{1}, i_{2}\right)$, then the two faces $S_{i_{1}}$ and $S_{i_{2}}$ have an $\left(n_{0}-2\right)$-dim face in common, call it $S_{i_{1} i_{2}}$. If $M\left(i_{1}, i_{2}, i_{3}\right) \neq \varnothing$, then we have an $\left(n_{0}-3\right)$-dim edge $S_{i_{1} i_{2} i_{3}}$ corresponding to where the three $\left(n_{0}-2\right)$-dim edges $S_{i_{1} i_{2}}, S_{i_{1} i_{3}}$ and $S_{i_{2} i_{3}}$ intersect, etc. Finally, each vertex $S_{i_{1}, \ldots, i_{n_{0}}}$ will correspond to $\left(M\left(i_{1}, \ldots, i_{n_{0}}\right)\right) \neq \varnothing$.

To see why we need $\Gamma$, let us again consider the example on pp. 136, 137 with $i_{0}=2, p=6$. Suppose for simplicity, $n_{0}=4$ and $\Gamma$ is a simple closed regular 3-dim tetrahedron (i.e., a cone on a 2-dim pyramid) with 5 3-dim faces $S_{i}, i=$ $1, \ldots, 5,102$-dim faces $S_{i j}, i, j=1, \ldots, 5, i \neq j, 10$ edges $S_{i j k}$ and 5 vertices $S: \ldots,$. 
Suppose the situation described on pp. 136,137 in the example is at vertex $S_{1234}$ with $S_{i}$ corresponding to $\left\{z_{i}=0\right\}=M(i)$.

Then $\gamma_{i_{0}+1}=\gamma_{3}$ is, near $S_{1234}$, given by $S_{124}+S_{123}$ and before constructing the tube $\tau_{3}$, we are considering, near $S_{1234}$, the 2-cycle (in $\Gamma$ ) $S_{13}+$ $S_{14}+S_{23}+S_{24} \approx \gamma_{4} \cap U$. (Note. $\approx$ will mean correspond to.) But $\gamma_{3}$ is a 2-cycle in $\bar{M}_{3}$ and $\bar{M}_{3} \approx \Gamma_{1}$, the 1-skeleton of $\Gamma$. Hence we must have a 1 -cycle in $\Gamma_{1}$, i.e., $S_{123}$ and $S_{124}$ have $S_{1235}$ and $S_{1245}$ (respectively) as vertices. Suppose that $\gamma_{3} \approx S_{123}+S_{124}+S_{125} \in Z_{1}\left(\Gamma_{1}\right) \subset Z_{1}(\Gamma)$. Then for $\gamma_{5}=\gamma_{p-1}$ to be a cycle, we have that, before constructing tubes, that $\gamma_{4}$ must also have $S_{15}+S_{25}$, i.e., $\gamma_{4} \approx S_{13}+S_{14}+S_{23}+S_{24}+S_{15}+S_{25} \in Z_{2}(\Gamma)$. That is, we start with $\gamma_{p-1}=C_{2 i_{0}-2} \times \tau_{i_{0}+1}\left(\gamma_{i_{0}+1}\right)$, but if we did not construct $\tau_{i_{0}+1}$, we have a $(p-2)$-dim cycle in $V$, which in $U$ is of the form $\sum_{i=1}^{2} \sum_{j=3}^{4} \pi_{4}(M(i, j))$. But as we approach $S_{5}$, if we assume $\gamma_{2} \approx S_{123}+S_{124}$ $+S_{125}$, then for $\gamma_{4}$ to remain a cycle, we must have $M(1,5)+M(2,5) \approx S_{15}+$ $S_{25}$. Now, $\partial M(3)=M(1,3)+M(2,3)-M(3,4)-M(3,5)$, but $M(5) \cap U=\varnothing$, so that $\partial M(3) \cap U$ does not have $M(3,5)$ as was in the example. In $\Gamma$, this corresponds to $\partial S_{3}=S_{13}+S_{23}-S_{34}-S_{35}$. Also $\partial S_{5}=S_{15}+S_{25}+S_{35}$ $+S_{45}$ so that $\gamma_{4} \approx S_{14}+S_{24}+S_{34}-S_{45}\left(=\partial S_{4}\right)$. But this is precisely a tubular element, i.e., in $V$, we have $M(1,4)+M(2,4)+M(4,3)-M(4,5)$ which is tubular, as at each 4-tuple point, we have exactly 3 4-dim edges intersecting. In $\Gamma$, at each vertex $S_{1234}, S_{1245}, S_{1345}$, and $S_{2345}$ of $\gamma_{4}$ we have exactly 3 edges, i.e., tubular 5-dim cycles of $V$ will correspond to simple closed 2-dim polygons of $\Gamma$ where a simple 2-dim polygon is one where at each vertex there are exactly 3 edges.

Thus, to show that the tubular cycles generate $R_{6}(V)$ is equivalent to showing that each 2-cycle of $\Gamma$ is homologous to a simple closed 2-dim polygon. But it is clear that $\Gamma$ is generated by such, i.e., $H_{3}(\Gamma) \approx Z$ with a generator $\sum_{i=1}^{5} S_{i}$ and the boundary of each $S_{i}$ is a simple closed 2-dim polygon.

This is precisely what happens in the global case:

Suppose we are given the representative $\gamma_{p-1}=\partial c_{p}, c_{p} \subset V$ and we let $k_{0}=\max _{k}\left\{c_{p} \cap M_{k} \neq \varnothing\right\}$. We have, of course, $k_{0}>i_{0}$ by the above remarks.

Case I. $\dot{n}_{0}=k_{0}$.

Then locally $\gamma_{p-1}=C_{2 i_{0}-2} \times \tau_{i_{0}+1}\left(\gamma_{i_{0}+1}\right)$ corresponds to a $\gamma_{p-2} \subset \bar{M}_{2}$ (before we construct $\tau_{i_{0}+1}$ ) which we saw before Lemma 2.10 is essentially $\sum_{i=1}^{i_{0}} \sum_{j=i_{0}+1}^{m} \pi_{m}(M(i, j))$. The $\gamma$ is unimportant to finding a tubular representative as we perform the homologies in $\pi_{m}(U)$.

$\gamma_{p-2}$ will then correspond to $\alpha$, an $\left(n_{0}-2\right)$-dim cycle of $\Gamma$ and $\gamma_{i_{0}+1}$ to an $\left(n_{0}-i_{0}-1\right)$-dim cycle of $\Gamma_{n_{0}-i_{0}-1}$, the $\left(n_{0}-i_{0}-1\right)$-skeleton of $\Gamma$. 
Namely, since $k_{0}=n_{0}$ and $\pi_{m}$ in Lemma 2.10 is independent of $m$, we can assume $\pi_{m}$ is never onto an $M(i)$, i.e., $\pi_{m}: \mathbf{C}^{n} \rightarrow \mathbf{C}^{n}$ and we can assume coordinates have been chosen so that for $i>m,\left\{z_{i}=0\right\} \not \subset V$. Then each $\pi_{m}(M(i, j))$ corresponds to an $\left(n_{0}-2\right)$-dim chain of $\Gamma$ and if $\gamma_{p-2}$ is a chain over which we can form a tube to get $\gamma_{p-1}$, then these $\left(n_{0}-2\right)$-dim chains must fit together to form an $\left(n_{0}-2\right)$-dim cycle of $\Gamma$. That is, if $\alpha$ is not a cycle, so that, say, at the vertex $\left(1, \ldots, n_{0}\right)$ we have $M(1,2) \subset \alpha$ but $M(1,3) \not \subset \alpha$ and $\partial M(1,2) \supset$ $M(1,2,3)$, then in $V \cap U$ where $U$ is a neighborhood and $V$ is given by $\Pi_{i=1}^{n} 0$ $=0$ in $U$, we cannot construct $\tau_{i_{0}+1}$ on $\gamma_{i_{0}+1}$.

Hence the proof of Lemma 2.10 is just a local manipulation in $\Gamma$ and to find a tubular cycle $\gamma_{p-2}^{\prime} \sim \gamma_{p-2}$ with $\gamma_{p-1}^{\prime}=\tau_{2}\left(\gamma_{p-2}^{\prime}\right) \sim \gamma_{p-1}$ in $V$ with $\tau_{1} \gamma_{p-1}^{\prime}$ $\sim \tau_{1} \gamma_{p-1}$ in $W-V$ is equivalent to finding a simple closed $\left(n_{0}-2\right)$-dim polygon $\alpha^{\prime} \sim \alpha$ in $\Gamma$, where $\alpha^{\prime}$ is a simple $\left(n_{0}-2\right)$-dim polygon if at each vertex there are

$$
\left(n_{0}-1\right) \text { edges, }\left(\begin{array}{c}
n_{0}-1 \\
2
\end{array}\right)-2 \cdot \operatorname{dim} \text { faces, } \ldots,\left(n_{0}-1\right)\left(n_{0}-2\right)-\operatorname{dim} \text { faces. }
$$

This is because $C_{n_{0}-1}(\Gamma)$ is generated by the $S_{i}$ which correspond to $M(i)$ so that $\alpha^{\prime} \sim \alpha$ in $\Gamma$ will correspond to $\gamma_{p-2}^{\prime} \sim \gamma_{p-2}$ in $V$ as shown in Lemma 2.10. Furthermore the condition that $\alpha^{\prime}$ is simple is precisely the condition of $\gamma_{p-2}^{\prime}$ being tubular, i.e., at each vertex $\alpha^{\prime}$ will have exactly $\left(n_{0}-1\right)$ edges, but if a vertex is given by $S_{1, \ldots, n_{0}}$, then the $\left(n_{0}-1\right)$ edges are given by, say, $S_{1, \ldots, \hat{i}_{1}, \ldots, n_{0}}$ for $i=1, \ldots, n_{0}-1$ (i.e., one of the indices must appear in each edge). This implies that the $\left(n_{0}-1\right)\left(n_{0}-2\right)$-dim faces of $\alpha^{\prime}$ at $S_{1, \ldots, n_{0}}$ are given by $S_{i n_{0}}$, $i=1, \ldots, n_{0}-1$. This corresponds to having $\gamma_{p-2}^{\prime}$ represented by $\Sigma_{i<n_{0}} \pi_{m}\left(M\left(i, n_{0}\right)\right) \times \gamma$ at $M\left(1, \ldots, n_{0}\right)$ which is a tubular element, so that $\tau_{2, n_{0}}\left(\Sigma_{i<n_{0}} \pi_{m}\left(M\left(i, n_{0}\right)\right) \times \gamma\right) \subset M_{1}$.

However, it is clear that $H_{n_{0}-1}(\Gamma)$ is a free abelian group with say $N$ generators $\beta_{i}, i=1, \ldots, N$, with each $\beta_{i}$ having as a representative a simple closed $\left(n_{0}-1\right)$-dim polygon $\Sigma_{j} S_{i_{j}}$. Also, $H_{n_{0}-2}(\Gamma)=0$ and $\partial S_{i}$ is a simple closed $\left(n_{0}-2\right)$-dim polygon. So that given $\alpha \in Z_{n_{0}-2}(\Gamma)$, it is easy to see that $\exists \alpha^{\prime}$ with $\alpha^{\prime} \sim \alpha$ and $\alpha^{\prime}$ a simple closed $\left(n_{0}-2\right)$-dim polygon. (One can give a precise proof of this by induction on the number of faces $S_{i}$ of $\Gamma$.)

Case II: $k_{0}<n_{0}$.

Again recall we have $\gamma_{p-1}=\partial c_{p}, c_{p} \subset V$. Let

$$
I(k)=\left\{\left(i_{1}, \ldots, i_{k}\right) \mid M\left(i_{1}, \ldots, i_{k}\right) \cap c_{p} \neq \varnothing\right\}
$$


Then $k_{0}=\max _{k}\{I(k) \neq \varnothing\}$, and let $\bigcup_{k} I(k)=I$. Form $\Gamma_{I}$, a $\left(k_{0}-1\right)$-dim polyhedron corresponding to $I$ : if $i \in I(i)$ then we take an $\left(k_{0}-1\right)$-dim face, called $S_{i}$. If $(i, j) \in I(2)$, then $S_{i}$ and $S_{j}$ have a $\left(k_{0}-2\right)$-dim face $S_{i j}$ in common, etc.

Then as in the case $k_{0}=n_{0}$, we can form $\alpha$, a $\left(k_{0}-2\right)$-dim cycle in $\Gamma_{I}$ which correspond to $\gamma_{p-2}$. We then have by induction on $k_{0}$, that $\alpha \sim \alpha^{\prime}$, a simple closed $\left(k_{0}-2\right)$-dim polygon.

Now, if $\prod_{i=1}^{k_{0}} z_{i}$ defines $V$ in $U$ and $\gamma_{p-1}$ is given in $U$ so that

$$
\gamma_{p-2}=\sum_{i=1}^{i_{0}} \sum_{j=i_{0}+1}^{k_{0}} \pi_{k_{0}}(M(i, j)) \times \gamma
$$

then $\partial \pi_{k_{0}}(M(i))=\Sigma_{j<k_{0}} \pm \pi_{k_{0}}(M(i, j))$. That is, the changes we make in $\Gamma_{I}$ correspond to homologous changes in $V$ to form $\gamma_{p-2}^{\prime}$, i.e., $\pi_{k_{0}}$ is a local change in $U$ but the changes in $\Gamma_{I}$ are global changes in $V$ which give a consistent local change in each $U$. In $\Gamma_{I}$, we have $\partial S_{i}=\Sigma_{j} \pm S_{i j}$ where $S_{j}$ corresponds to $\pi_{k_{0}}(M(i))$, and $S_{i j}$ to $\pi_{k_{0}}(M(i, j))$. Thus, we have the same situation as on $\Gamma$ when $n_{0}=k_{0}$.

Note that what we are doing can be seen in another context as follows:

We can embed $\Gamma_{I} \subset \Gamma$ as a subcomplex of the first barycentric subdivision of $\Gamma$ :

If $\left(i_{1}, \ldots, i_{k_{0}}\right)_{\Gamma_{I}}$ is a vertex of $\Gamma_{I}$, then at the $\left(n_{0}-k_{0}\right)$-dim face $\left(i_{1}, \ldots, i_{k_{0}}\right) \subset \Gamma$ (i.e., $\left.S_{i_{1}, \ldots, i_{k_{0}}}\right)$, we take the barycenter to correspond to the vertex. If $\left(i_{1}, \ldots, \hat{i}_{j}, \ldots, i_{k_{0}}\right)_{\Gamma_{I}}$ is an edge of $\Gamma_{I}$ joining $\left(i_{1}, \ldots, i_{k_{0}}\right)_{\Gamma_{I}}$ to, say, $\left(i_{1}, \ldots, i_{j}^{\prime}, \ldots, i_{k_{0}}\right)_{\Gamma_{I}}$, then in the $\left(n_{0}-k_{0}+1\right)$-dim face $\left(i_{1}, \ldots, \hat{i}_{j}, \ldots, i_{k_{0}}\right)_{\Gamma} \subset \Gamma$, we join the barycenter of $\left(i_{1}, \ldots, i_{k_{0}}\right)_{\Gamma}$ and $\left(i_{1}, \ldots, i_{j}^{\prime}, \ldots, i_{k_{0}}\right)_{\Gamma}$ to form an edge. We continue in this fashion.

Then under the embedding, $\alpha \sim \widetilde{\alpha}$, a $\left(k_{0}-2\right)$-dim cycle of the first barycentric subdivision of $\Gamma$.

Also, note that $\Gamma_{I} \subset \Gamma-\Gamma_{n_{0}-k_{0}-1}$, i.e., $\Gamma_{I}$ misses the $\left(n_{0}-k_{0}-1\right)$ dim skeleton of $\Gamma$ and $\Gamma_{k_{0}}$ is a deformation retract of $\Gamma-\Gamma_{n_{0}-k_{0}-1}$ (as $\operatorname{dim}_{R} \Gamma=n_{0}-1$ and $\left.n_{0}-1-\left(n_{0}-k_{0}-1\right)-1=k_{0}-1\right)$. We can deform $\Gamma_{I}$ into $\Gamma_{k_{0}-1}$ as a subpolyhedron which is PL-homeomorphic to the original $\Gamma_{I}$. Then we work in $\Gamma_{k_{0}-1}$ to find $\alpha^{\prime}$ and then push everything back into $\Gamma$ $\Gamma_{n_{0}-k_{0}-1}$. Q.E.D. for Corollary 2.8 .

2.11 Definition. We say $V \subset W$ has local normal crossings if $V$ is locally given by $\prod_{i=1}^{k} z_{i}^{a}{ }_{i}=0$ where $\left(z_{1}, \ldots, z_{n}\right)$ are local coordinates. 
The proof of Theorem 2.2 is still valid in the local normal crossings case. In fact, a more general result is proven in $\$ 4$ for arbitrary $V$. Corollary 2.8 is also valid but it takes some work to prove it. The induction should be changed to an induction on $\operatorname{dim} V$ and $n_{0}$, where $n_{0}=\max _{k}\left\{\Pi_{i=1}^{k} z_{i}^{a_{i}}=0\right.$ defines $\left.V\right\}$, The sole difficulty in proving the result is the global construction of $\Gamma$ (i.e., Lemma 2.10 is a local result) and here we can form $\Gamma$ which is no longer a polygon but a simplicial complex. The $S_{i}$, the $\left(n_{0}-1\right)$-dim simplex of $\Gamma$ would correspond to the global components of $V$, e.g. we can have a component intersect itself many times at a point. Also, when $S_{i}$ intersects itself, we would form $S_{i j_{k}}$ where $j$ could equal $i$, e.g., $S_{11_{1}}, S_{11_{2}}$, etc, and the $S_{i j_{k}}$ would correspond to the global components of $\bar{M}_{2}$, a subvariety of $V$. Again, if $M(i)$ and $M\left(i, j_{k}\right)$ are the components of $V$ and $\bar{M}_{2}$ respectively, then homology in $V$ via $\partial M(i)=$ $\Sigma_{j} M\left(i, j_{k}\right)$ would correspond to homology in $\Gamma$ via $\partial S(i)$. Then the difficult part will be to give a precise proof that simple closed $\left(n_{0}-2\right)$-dim simplices are in each equivalence class of homology. However, this can be done by, e.g. a double induction on the number of components and $n_{0}$. (For $n_{0}=2$, first step in the induction, we have a graph and results are clear.) The details are left to the reader.

To get the relations among the various geometric $q$-residues, let us say that two geometric $q$-residues $\gamma_{p}, \gamma_{p}^{\prime}$ are equivalent $\left(\gamma_{p} \sim \gamma_{p}^{\prime}\right)$ if $\gamma_{p}-\gamma_{p}^{\prime}=\partial c_{p+1}$. $c_{p+1} \subset V$ such that $\tau_{1}\left(\gamma_{p}\right)-\tau_{1}\left(\gamma_{p}^{\prime}\right)=-\partial\left(\tau_{1}\left(c_{p+1}\right)\right)$. This is clearly an equivalence relation.

2.12 Definition. Let $H_{p-1}(V)_{\Delta}$ denote

$$
H_{p-1}\left(\bar{M}_{1}\right)_{\Delta} \oplus \tau_{2} H_{p-2}\left(\bar{M}_{2}\right)_{\Delta} \oplus \cdots \oplus \tilde{\tau}_{2} \cdots \tilde{\tau}_{p-1} \tau_{p} H_{0}\left(\bar{M}_{p}\right)_{\Delta}
$$

modulo the equivalence relation.

Then $\tau_{1}=\tau: H_{p-1}(V)_{\Delta} \rightarrow H_{p}(W-V)$.

2.13 Proposition. The sequence

$$
H_{p+1}(W) \stackrel{I}{\rightarrow} H_{p-1}(V)_{\Delta} \stackrel{\tau}{\rightarrow} H_{p}(W-V)
$$

is exact where $I$ is intersection with $V$, i.e., essentially cap product with the dual cohomology class of $V$.

PRoof. Let $\gamma_{p+1} \in H_{p+1}(W)$. Then $I\left(\gamma_{p+1}\right)=\gamma_{p+1} \cap V=\gamma_{p-1}$. But by the construction in Corollary 2.8, $\tau\left(\gamma_{p-1}\right)=\partial\left(\gamma_{p+1}-T \gamma_{p-1}\right)$ where $T$ is the solid tube over $\gamma_{p-1}$, i.e., all fibres of length less than or equal to $\epsilon$. Therefore $\tau \cdot I=0$.

Conversely, suppose $\gamma_{p-1} \in H_{p-1}(V)_{\Delta}$ and $\tau\left(\gamma_{p-1}\right)=\partial c_{p+1}$ for $c_{p+1} \subset$ 
$W-V$. Then set $\gamma_{p+1}=T \gamma_{p-1}-c_{p+1}$. But $\partial \gamma_{p+1}=\tau\left(\gamma_{p-1}\right)-\tau\left(\gamma_{p-1}\right)=0$, so that $\gamma_{p+1} \in H_{p+1}(W)$ and clearly $I\left(\gamma_{p+1}\right)=\gamma_{p-1}$. Q.E.D. for Proposition 2.13 .

Therefore the relations are given by $I\left(H_{p+1}(W)\right)$. To say $I\left(\gamma_{p+1}\right)=0$ means that if

$$
I\left(\gamma_{p+1}\right)=\left(\gamma_{p-1}, \tau_{2}\left(\gamma_{p-2}\right), \ldots, \tilde{\tau}_{2} \cdots \tilde{\tau}_{p-1} \tau_{p} \gamma_{0}\right)
$$

for $\gamma_{p-j} \in H_{p-j}\left(\bar{M}_{j}\right)_{\Delta}$ then each $\gamma_{p-j}$ is homologous to zero in $H_{p-j}\left(\bar{M}_{j}\right)$, i.e., $\gamma_{p-j}=\partial c_{p-j+1}$ and $c_{p-j+1} \subset \bar{M}_{j}$. In fact $\tilde{\tau}_{1} \cdots \tilde{\tau}_{j-1} c_{p-j+1}$ is a $(p+1)$ chain in $W-V$ whose boundary is $\tilde{\tau}_{1} \cdots \widetilde{\tau}_{j-1} \tau_{j} \gamma_{p-j}$ by the definition of homologous in $H_{p-1}\left(V_{1}\right)_{\Delta}$.

2.14 Corollary. The following is exact:

$$
\cdots \rightarrow H_{p+1}(W) \stackrel{I}{\rightarrow} H_{p-1}(V)_{\Delta} \stackrel{\tau}{\rightarrow} H_{p}(W-V) \stackrel{i}{\rightarrow} H_{p}(W) \rightarrow \cdots .
$$

PRoof. Exactness at $H_{*}(V)_{\Delta}$ is Proposition 2.11, while exactness at $H_{*}(W-V)$ is essentially Corollary 2.8. By the above discussion we have exactness at $H_{*}(W)$ Q.E.D.

We would like to note here that Theorem 2.2 and Corollary 2.8 are really a geometric investigation of a special sequence. Namely if we take the inclusion map of $W-V \subset W$ and look at the induced Leray spectral sequence, then

$$
E_{2}^{p, q} \simeq \sum_{\left\{i_{1}, \ldots, i_{q}\right\}} H^{p}\left(\bar{M}\left(i_{1}, \ldots, i_{q}\right)\right)
$$

and

$$
E_{2}^{p, q} \Rightarrow H^{p+q}(W-V) .
$$

(See Hodge-Atiyah [11] for a detailed investigation of this sequence. However, they use coefficients in C.) Then $d_{2}^{p, q}$ is just the Gysin map and $\operatorname{Ker} d_{2}^{p, q}=$ $H^{p}\left(\bar{M}_{q}, \mathrm{C}\right)_{\Delta}$. Also see Gordon [5] for a further discussion of this spectral sequence. We also note that Deligne [4] has generalized these results to the case of schemes over $\mathbf{C}$. For a detailed investigation of $V$ in the general case see the end of $\S 4$.

3. More remarks about the normal crossings case. In the previous section we investigated the problem of the residues of $V$ when $V$ has normal crossings. We will now show that this is quite sufficient in most cases and also give a further topological reduction if further hypotheses are satisfied.

3.1 Proposition. If $W^{\prime}$ is obtained from $W$ by a monoidal transform $\pi$ whose center $X \subset V$ is nonsingular and if $\pi^{-1}(V)=V^{\prime}$, then $\pi_{*}: R_{p}\left(V^{\prime}\right) \simeq R_{p}(V)$. 
Proof. From the commutative diagram

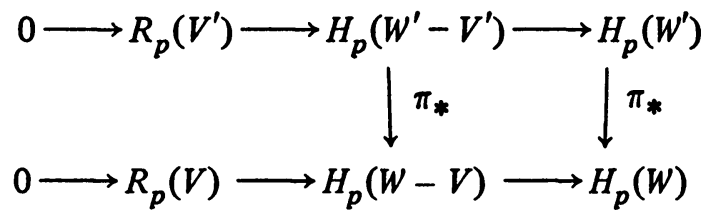

we have that $\pi_{*} R_{p}\left(V^{\prime}\right) \subseteq R_{p}(V)$.

Suppose $\alpha \in R_{p}(V)$. Then $\alpha$ has a representative $\gamma_{p}$ such that $\gamma_{p}=\partial C_{p+1}$, $C_{p+1} \subset W$ with $C_{p+1}$ intersecting $X$ transversely, i.e., if $\operatorname{codım}_{\mathrm{C}} X=m$, then $C_{p+1} \cap X=\gamma_{p+1-2 m}$, and if $N(X)$ is a normal disk bundle of $X$ in $W$, then, locally, $C_{p+1} \cap N(X)=\gamma_{p+1-2 m} \times D_{2 m}$ where $D_{2 m}$ is a $2 m$-dim disk.

The difficulty with having $\left(\pi_{*}\right)^{-1} \alpha \in R_{p}\left(V^{\prime}\right)$ is that if we let $\gamma_{p}^{\prime}=$ $\pi^{-1}\left(\gamma_{p}\right), C_{p+1}^{\prime}=\pi^{-1}\left(C_{p+1} \cap(W-V)\right)$ and $C^{\prime}$ be the closure of $C_{p+1}^{\prime}$ in $W^{\prime}$, then $C^{\prime} \cap V$ may not be a $(p-1)$-dim chain, i.e., it may be too large.

Set theoretically, we have $W^{\prime}=(W-X) \cup_{X} P N(X)$, where $P N(X)$ is the projective-normal bundle of $X$ in $W$ with fibre $\mathbf{C} P_{m-1}$. Then let $U$ be a neighborhood of $W$ with local coordinates $\left(x_{1}, \ldots, x_{n}\right)$ and $\left\{x_{1}=\cdots=x_{m}=0\right\}=$ $X \cap U$ and let $\mathbf{C} P_{m-1}=\left[X_{1}, \ldots, X_{m}\right]$ in homogeneous coordinates. Then $W^{\prime} \cap \pi^{-1}(U)=\bar{\Gamma} \subset U \cap \mathbf{C} P_{m-1}$ where

$$
\Gamma=\left\{\left(x_{1}, \ldots, x_{n}\right) \times\left[X_{1}, \ldots, X_{m}\right] \mid x_{i} X_{j}=x_{j} X_{i}, i \leqslant i, j \leqslant m\right\}
$$

Hence, locally, $C_{p+1} \cap U=\mathbf{C}_{1} \times \cdots \times \mathbf{C}_{m} \times\left(\gamma_{p+1-2 m} \cap U\right)$ and $C^{\prime} \cap$ $\pi^{-1}(U) \cap V^{\prime}=C P_{m-1} \times \gamma_{p+1-2 m}^{\prime}$ where $\gamma_{p+1-2 m}^{\prime}$ is a $(p+1-2 m)$-dim chain in $\pi^{-1}(U)$ which is isomorphic to $\gamma_{p+1-2 m} \cap U$. Thus $C^{\prime} \cap V^{\prime}$ is a $(p-1)$-dim chain, i.e., $C^{\prime}$ is a $(p+1)$-dim chain with $\partial C^{\prime}=\gamma_{p}^{\prime}$. Hence $\pi_{*}^{-1} \alpha \in R_{p}\left(V^{\prime}\right)$. Q.E.D. for Proposition 3.1.

Hironaka [9] has shown that if $W$ is algebraic or a Stein manifold (in fact, any closed submanifold of a (Stein variety) $\times$ (algebraic variety)), then, if $V$ is a hypersurface of $W$, after a finite number of monoidal transforms with nonsingular centers contained in $V$, we can obtain $\pi: W^{\prime} \rightarrow W$, also algebraic if $W$ is, such that $V^{\prime}=\pi^{-1}(V)$, the total transform, has normal crossings in the sense of Definition 2.1.

Then recently Lejeune-Teissier [13] have shown that if $W$ is a reduced complex analytic space and if $V \subset W$ is a hypersurface, one can (by a finite number of monoidal transforms centered on nonsingular parts of $V$ ) obtain $W^{\prime}$ with $V^{\prime}$ having normal crossings..

Hence, in the above cases, the results of $\$ 2$ are valid.

Next we want to make some further topological investigations of $R_{p}(V)$ 
when $V$ has normal crossings. Recall that in Corollary 2.8. we showed that $R_{p}(V)$ is generated by cycles of the form $\tilde{\tau}_{1} \cdots \tilde{\tau}_{q-1} \tau_{q}\left(\gamma_{p-q}\right)$ for $\gamma_{p-q} \in$ $H_{p-q}\left(M_{q}\right)_{\Delta}$. If $\gamma_{p-q} \in H_{p-q}\left(M_{q}\right)_{\Delta}$ and $\gamma_{p-q}=\Sigma \gamma_{p-q, i_{1}, \ldots, i_{q}}$ then we first want to consider $\tau_{p-q, i_{j}}\left(\gamma_{p-q, i_{1}, \ldots, i_{q}}\right)$ in $\bar{M}\left(i_{1}, \ldots, \hat{i}_{j}, \ldots, i_{q}\right)-$ $\bar{M}\left(i_{1}, \ldots, i_{q}\right)$. For simplicity assume $q=1$.

3.2 PRoposition. Let $\gamma_{p-1}=\Sigma_{i \in I} \gamma_{p-1, i} \in H_{p-1}\left(\bar{M}_{1}\right)_{\Delta}$.

(a) If $\gamma_{p-1, i_{0}} \sim 0$ in $V_{i_{0}}$, then $\tau_{1}\left(\gamma_{p-1}\right) \sim \tau_{1}\left(\gamma_{p-1}^{\prime}\right)$ in $W-V$, where $\gamma_{p-1}^{\prime}=\Sigma_{i \in I ; i \neq i_{0}} \gamma_{p-1, i}^{\prime}$ and $\gamma_{p-1, i}^{\prime} \sim \gamma_{p-1, i}$ in $V_{i}$.

(b) If $\tau_{1, i_{0}}\left(\gamma_{p-1, i_{0}}\right) \sim 0$ in $W-V_{i_{0}}$, then $\tau_{1}\left(\gamma_{p-1}\right) \sim \tau_{1}\left(\gamma_{p-1}^{\prime}\right)$ where $\gamma_{p-1}^{\prime}=\Sigma_{i \neq i_{0}} \gamma_{p-1, i}^{\prime}$.

Furthermore, if $\tau_{1, i_{0}}\left(\gamma_{p-1, i_{0}}\right) \sim 0$ in $W-\left(V_{i_{0}} \cup \bigcup_{j \notin I} V_{j}\right)$, then $\tau_{1}\left(\gamma_{p-1}\right)$ $\sim \tau_{1}\left(\gamma_{p-1}^{\prime}\right)$ where $\gamma_{p-1}=\Sigma_{i \neq i_{0} ; i \in I} \gamma_{p-1, i}^{\prime}$. Also, if $\tau\left(\gamma_{p-1, i_{0}}\right) \sim 0$ in $W-$ $V_{i_{1}}\left(\right.$ or $\left.W-\left(V_{i_{1}} \cup \bigcup_{j \notin I} V_{j}\right)\right)$ then $\tau_{1}\left(\gamma_{p-1, i_{1}}^{\prime}\right) \sim 0$ in $W-V_{i_{1}}$ (or $W-$ $\left.\left(V_{i} \cup \bigcup_{j \notin I} V_{j}\right)\right)$.

PRoof. (a) Suppose $i_{3}=1$. Then if $\gamma_{p-1,1} \sim 0$ in $V_{1}$, then $\gamma_{p-1,1}=$ $\partial c_{p, 1}$ for $c_{p, 1} \subset V_{1}$. Let $c_{p-2,1 i}=c_{p, 1} \cap V_{i}$ where $c_{p-2,1 i}$ is a $(p-2)$ chain in $V_{1} \cap V_{i}$, since $V_{i}$ intersects $V_{1}$ transversely. Then we have

$$
\gamma_{p-3,1 i}=\gamma_{p-1,1} \cap V_{i}=\gamma_{p-1, i} \cap V_{1}=\partial\left(c_{p-2,1 i}\right) .
$$

Put

$$
\gamma_{p-1, i}^{\prime}=\gamma_{p-1, i}-T_{2,1}\left(\gamma_{p-3,1 i}\right)-T_{2,1}\left(c_{p-2,1 i}\right)
$$

for $i \neq 1$. Note that

$$
\partial T_{2,1} c_{p-2,1 i}=\tau_{2,1} c_{p-2,1 i}+T_{2,1} \gamma_{p-3,1 i} .
$$

If we set $\tilde{c}_{p, 1}=c_{p, 1}-\Sigma_{i \neq 1} T_{1, i}\left(c_{p-2,1 i}\right)$ then $\tau_{1,1}\left(\tilde{c}_{p, 1}\right) \subset W-V$ and

$$
\begin{aligned}
\partial \tau_{1,1}\left(\tilde{c}_{p, 1}\right) & =-\tau_{1,1}\left(\partial \tilde{c_{p, 1}}\right) \\
& =\sum_{i>1}\left\{\tau_{1,1} \tau_{2,1} c_{p-2,1 i}+\tau_{1,1} \tau_{2, i} \gamma_{p-3,1 i}\right\}-\tau_{1, i} \tilde{\gamma}_{p-1,1} .
\end{aligned}
$$

This implies

$$
\sum_{i>1} \tau_{1, i}\left(\tilde{\gamma}_{p-1, i}^{\prime}\right)=\sum_{i \geqslant 1} \tau_{1, i}\left(\tilde{\gamma}_{p-1, i}\right)+\partial \tau_{1,1}\left(\tilde{c}_{p, 1}\right) .
$$

Thus $\Sigma_{i>1} \tau\left(\gamma_{p-1, i}^{\prime}\right) \sim \Sigma_{i \geqslant 1} \tau\left(\gamma_{p-1, i}\right)$ in $W-V$ and $\gamma_{p-1, i}^{\prime} \sim \gamma_{p-1, i}$ in $V_{i}$ for $i>1$. 
(b) Again suppose $i_{0}=1$ and $\tau_{1,1}\left(\gamma_{p-1,1}\right) \sim 0$ in $W-V_{1}$.

Then $\tau_{1,1}\left(\gamma_{p-1,1}\right)=\partial c_{p+1}$ for $c_{p+1,1} \subset W-V_{1}$. Let $c_{p+1,1} \cap V_{i}=$ $\Gamma_{p-1, i}^{1} \subset V_{i}-V_{i} \cap V_{1}$ for $i>1$.

$$
\begin{aligned}
\partial \Gamma_{p-1, i}^{1} & =\left(\partial c_{p+1}\right) \cap V_{i}=\left[\tau_{1,1}\left(\gamma_{p-1,1}\right)\right] \cap V_{i} \\
& =\tau_{2,1}\left(\gamma_{p-1,1} \cap V_{i}\right)=\tau_{2,1}\left(\gamma_{p-3,1 i}\right)
\end{aligned}
$$

where $\gamma_{p-3,1 i}=\gamma_{p-1,1} \cap V_{i}=\gamma_{p-1, i} \cap V_{1}$. The second from last equality follows because we can choose the normal bundle of $V_{1}$ with respect to $V_{i}$ so that

$$
\tau_{1,1} \mid V_{1} \cap V_{i}=\tau_{2,1}: V_{1} \cap V_{i} \rightarrow V_{i}-V_{1} \cap V_{i}
$$

by definition of $\tau_{2,1}$.

Then set $\gamma_{p-1, i}^{\prime}=\tilde{\gamma}_{p-1, i}+\Gamma_{p-1, i}^{1}$ for $i>1$ where $\tilde{\gamma}_{p-1, i}=\gamma_{p-1, i}-$ $T_{2,1}\left(\gamma_{p-3,1 i}\right) . \partial \gamma_{p-1, i}^{\prime}-\tau_{2,1} \gamma_{p-3,1 i}+\tau_{2,1} \gamma_{p-3,1 i}=0$ by Lemma 2.5. Now,

$$
\begin{aligned}
\partial\left(\sum_{i>1} T_{1, i} \Gamma_{p-1, i}^{1}\right) & =\sum_{i>1} \tau_{1, i} \Gamma_{p-1, i}^{1}+\sum_{i>1} T_{1, i} \tau_{2,1} \gamma_{p-3,1 i} \\
& =\sum_{i>1} \tau_{1, i} \Gamma_{p-1, i}^{1}+\sum_{i>1} \tau_{1,1} T_{2, i} \gamma_{p-3,1 i}
\end{aligned}
$$

since $T_{1, i} \tau_{2,1}=\tau_{1,1} T_{2, i}$ by their definition (i.e., $\partial\left(D^{2} \times S^{1}\right)=\partial\left(S^{1} \times D^{2}\right)$ so that as oriented chains, $\left.D^{2} \times S^{1}=S^{1} \times D^{2}\right)$. Also $\Sigma_{i>1} T_{2, i} \gamma_{p-3,1 i}=$ $\gamma_{p-1,1}-\tilde{\gamma}_{p-1,1}$, so that

$$
\partial\left(\sum_{i>1} T_{1, i} \Gamma_{p-1, i}^{1}\right)=\sum_{i>1} \tau_{1, i} \Gamma_{p-1, i}^{1}+\tau_{1,1}\left(\gamma_{p-1,1}-\tilde{\gamma}_{p-1,1}\right)
$$

Thus

$$
\partial\left(c_{p+1}-\sum_{i>1} T_{1, i} \Gamma_{p-1, i}^{1}\right)=\tau_{1, i}\left(\tilde{\gamma}_{p-1,1}\right)-\sum_{i>1} \tau_{1, i} \Gamma_{p-1, i}^{1} .
$$

Hence

$$
\sum_{i>1} \tau_{1, i}\left(\gamma_{p-1, i}^{\prime}\right)=\sum_{i \geqslant 1} \tau_{1, i}\left(\gamma_{p-1, i}\right)-\partial\left(c_{p+1}-\sum_{i>1} T_{1, i} \Gamma_{p-1, i}^{1}\right),
$$

and $c_{p+1}-\Sigma_{i>1} T_{1, i} \Gamma_{p-1, i}^{1}$ is a $(p+1)$-chain in $W-V_{1}$.

Furthermore, note that if $c_{p+1} \cap V_{j}=\varnothing$, then $\gamma_{p-1, j}^{\prime}=\gamma_{p-1, j}$, so that this proves the second part of $(b)$.

Finally, suppose that $\tau_{1, i_{1}}\left(\gamma_{p-1, i_{1}}\right) \sim 0$ in $W-V_{i_{1}}$ (or $W-\left(V_{i_{1}} \cup\right.$ $\left.\bigcup_{j \notin I} V_{j}\right)$ ). For simplicity suppose $i_{1}=2$. Then if $\gamma_{p-3,12}=\gamma_{p-1,1} \cap V_{2}=$ $\gamma_{p-1,2} \cap V_{1}$, we have that 


$$
\begin{aligned}
T_{1,2} \tau_{2,1} \gamma_{p-3,12} & =-T_{1,1} \tau_{2,2} \gamma_{p-3,12}+\partial T_{1,1} T_{2,2} \gamma_{p-3,12} \\
& =-\tau_{1,2} T_{2,1} \gamma_{p-3,12}+\partial T_{1,1} T_{2,2} \gamma_{p-3,12} \\
& =-\tau_{1,2}\left(\gamma_{p-1,2}-\tilde{\gamma}_{p-1,2}\right)+\partial T_{1,1} T_{2,2} \gamma_{p-3,12},
\end{aligned}
$$

and

$$
\begin{aligned}
\tau_{1,1} \gamma_{p-1,1}= & \tau_{1,1}\left(\tilde{\gamma}_{p-1,1}\right)+\tau_{1,1}\left(\gamma_{p-1,1}-\tilde{\gamma}_{p-1,1}\right) \\
= & \partial T_{1,1} \tilde{\gamma}_{p-1,1}-T_{1,1}\left(\partial \tilde{\gamma}_{p-1,1}\right)+\tau_{1,1}\left(\gamma_{p-1,1}-\tilde{\gamma}_{p-1,1}\right) \\
= & \partial T_{1,1} \tilde{\gamma}_{p-1,1}+T_{1,1} \tau_{2,2} \gamma_{p-3,12} \\
& -T_{1,1} \tau_{2,2} \gamma_{p-3,12}+\partial T_{1,1} T_{2,2} \gamma_{p-3,12} \\
= & \partial T_{1,1} \tilde{\gamma}_{p-1,1}+\partial T_{1,1} T_{2,2} \gamma_{p-3,12}
\end{aligned}
$$

where the third equality follows from Lemma 2.5 and the previous sequence of equalities.

Hence, using the above two sets of equalities we have

$$
\begin{aligned}
& \partial\left(c_{p+1,1}-T_{1,2} \Gamma_{p-1,2}^{1}\right) \\
& \quad=\tau_{1,1} \gamma_{p-1,1}-\tau_{1,2} \Gamma_{p-1,2}^{1}-T_{1,2} \tau_{2,1} \gamma_{p-3,12} \\
& \quad=\partial T_{1,1} \tilde{\gamma}_{p-1,1}-\tau_{1,2} \Gamma_{p-1,2}^{1}+\tau_{1,2}\left(\gamma_{p-1,2}-\tilde{\gamma}_{p-1,2}\right) .
\end{aligned}
$$

So, if we have $\tau_{1,2} \gamma_{p-1,2}=\partial c_{p+1,2}$ for $c_{p+1,2} \subset W-V_{2}$ (or $W-$ $\left.\left(V_{2} \cup \cup_{j \notin I} V_{j}\right)\right)$, then

$$
\begin{aligned}
\tau_{1,2}\left(\gamma_{p-1,2}^{\prime}\right) & =\tau_{1,2}\left(\tilde{\gamma}_{p-1,2}\right)+\tau_{1,2} \Gamma_{p-1,2} \\
& =\partial\left(c_{p+1,2}\right)-\tau_{1,2}\left(\gamma_{p-1,2}-\tilde{\gamma}_{p-1,2}\right)+\tau_{1,2} \Gamma_{p-1,2}^{1} \\
& =\partial\left(c_{p+1,2}\right)+\partial T_{1,1} \tilde{\gamma}_{p-1,1}-\partial\left(c_{p+1,1}-T_{1,2} \Gamma_{p-1,2}^{1}\right)
\end{aligned}
$$

Also

$$
c_{p+1,2}+T_{1,1} \tilde{\gamma}_{p-1,1}-\left(c_{p+1,1}-T_{1,2} \Gamma_{p-1,2}^{1}\right) \subset W-V_{2}
$$

(or $W-\left(V_{2} \cup \cup_{j \notin I} V_{j}\right)$ if $c_{p+1,1}$ and $c_{p+1,2}$ also are contained in $W-$ $\left.\left(V_{2} \cup \cup_{j \notin I} V_{j}\right)\right)$. Q.E.D.

Note. We cannot say just because for all $i \in I, \tau_{1, i}\left(\gamma_{p-1, i}\right) \sim 0$ in $W-$ $\left(V_{i} \cup \cup_{j \notin I} V_{j}\right)$ that $\tau_{1}\left(\gamma_{p-1}\right) \sim 0$ in $W-V$ because 


$$
\begin{aligned}
\left(c_{p+1,2}+T_{1,1} \tilde{\gamma}_{p-1,1}-c_{p+1,1}+T_{1,2} \Gamma_{p-1,2}^{1}\right) & \cap V_{1} \\
& =\Gamma_{p-1,1}^{2}+\tilde{\gamma}_{p-1,1}=\gamma_{p-1,1}^{\prime}
\end{aligned}
$$

where $\Gamma_{p-1,1}^{2}=c_{p+1,2} \cap V_{1}$. However, we do have

3.3 Proposition. Let $\gamma_{p-1}=\Sigma_{i \in I} \gamma_{p-1, i} \in H_{p-1}\left(\bar{M}_{1}\right)_{\Delta}$. Then $\tau\left(\gamma_{p-1}\right)$ $\sim 0$ in $W-V$ if and only if

(i) $\tau_{1, i}\left(\gamma_{p-1, i}\right) \sim 0$ in $W-\left(V_{i} \cup \bigcup_{j \notin I} V_{j}\right)$;

(ii) if $\Gamma_{p+1, i} \subset W-\bigcup_{j \notin I} V_{j}$ with $\Gamma_{p+1, i} \cap V_{i}=\gamma_{p-1, i}$, then $\Gamma_{p+1, i}$ $\cap V_{k}=\gamma_{p-1, k}$ for all $k \in I$.

PRoof. $\Rightarrow$ Suppose $\tau_{1}\left(\gamma_{p-1}\right)=\Sigma_{i \in I} \tau_{1, i}\left(\tilde{\gamma}_{p-1, i}\right)=\partial c_{p+1} \subset W-V$. Then for $i_{0} \in I$, say $i_{0}=1$,

$$
\begin{aligned}
\partial\left(c_{p+1}-\sum_{i>1} T_{1, i} \tilde{\gamma}_{p-1, i}\right)= & \sum_{i \geqslant 1} \tau_{1, i} \tilde{\gamma}_{p-1,1}-\sum_{i>1} \tau_{1, i} \tilde{\gamma}_{p-1, i} \\
& +\sum_{i>1} T_{1, i} \tau_{2,1} \gamma_{p-3,1 i} \\
= & \tau_{1,1} \tilde{\gamma}_{p-1,1}+\sum \tau_{1,1} T_{2, i} \gamma_{p-3,1 i} \\
= & \tau_{1,1} \gamma_{p-1,1}
\end{aligned}
$$

and $\left(c_{p+1}-\Sigma_{i>1} T_{1, i} \tilde{\gamma}_{p-1, i}\right) \cap V_{j}=\varnothing$ for $j \notin I$ since $\gamma_{p-1, i} \cap V_{j}=\varnothing$ implies $T_{1, i}$ can be chosen so that $T_{1, i} \tilde{\gamma}_{p-1, i} \cap V_{j}=\varnothing$.

Also, notice that by the construction

$$
\Gamma_{p+1, i}=-c_{p+1}+\sum_{i>1} T_{1, i} \tilde{\gamma}_{p-1, i}+T_{1,1} \gamma_{p-1,1}
$$

so that

$$
\Gamma_{p+1,1} \cap V_{i}=+\tilde{\gamma}_{p-1, i}+T_{2,1} \gamma_{p-3,1 i}=\gamma_{p-1, i} .
$$

$\Leftarrow$ Conversely, if $\Gamma_{p+1,1} \cap V_{i}=\gamma_{p-1, i}$ for all $i \in I$, then $\gamma_{p-1}=$ $\Gamma_{p+1,1} \cap V$ so that $\tau\left(\gamma_{p-1}\right) \sim 0$ in $W-V$. Q.E.D.

If we are given $\tilde{\tau}_{1} \cdots \tilde{\tau}_{q-1} \tau_{q} \gamma_{p-q} \in R_{p}(V)$ with $\gamma_{p-q} \in H_{p-q}\left(\bar{M}_{q}\right)_{\Delta}$, then when can we guarantee $\gamma_{p-q} \in H_{p-q}\left(M_{q}\right)$, i.e., $\gamma_{p-q} \cap \bar{M}_{q+1}=\varnothing$ ? For if we had $\gamma_{p-q} \cap \bar{M}_{q+1}=\varnothing$, for all $q$, then this would considerably simplify many other problems involving residues, e.g., local invariant cycle problem (Griffiths [7, p. 249]).

There are facts which indicate this might be true i.e., $V$ ample in $W$ implies $R_{p}(V)$ is generated by $\gamma_{p-q} \in H_{p-q}\left(M_{q}\right) \subset H_{p-q}\left(\bar{M}_{q}\right)_{\Delta}$.

For example, if $V$ is ample and nonsingular, we have the commutative diagram: 


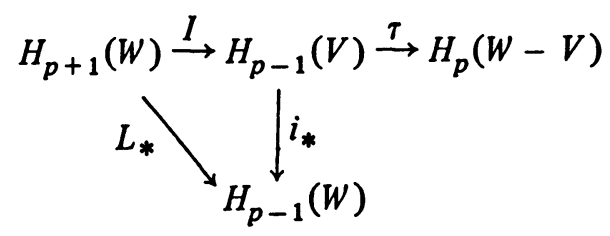

where $L_{*}$ is dual to the Hodge map, i.e., $L_{*}(\gamma)=\gamma \cap[V]^{*}$ with $[V]^{*}$ the dual two-dimensional cohomology class of $[V] \in H_{2 n-2}(W)$ and " $\cap$ " being cap product. If $V$ is ample, $L_{*}$ is surjective for $p \leqslant n$ (see Weil [26]), and by the Lefschetz theorem $i_{*}$ is an isomorphism for $p<n$ and surjective for $p=n$.

Thus for $p<n, I$ is surjective, hence $R_{p}(V)=0$. For $p=n$, since $H_{n-1}(W) \simeq H_{n+1}(W)$ by Poincaré duality (recall we have coefficients in $\mathrm{C}$ ), $L_{*}$ is an isomorphism, hence $\tau\left(\gamma_{n-1}\right) \nsim 0$ iff $\gamma_{n-1} \notin$ image $I$ iff $i_{*} \gamma_{n-1}=0$.

3.4 Proposition. $i_{*} \gamma_{n-1}=0$ implies

$$
\gamma_{n-1} \in \text { image }\left\{H_{n-1}\left(V-V \cap V^{\prime}\right) \rightarrow H_{n-1}(V)\right\}
$$

for any $V^{\prime}$ which is a nonsingular hypersurface of $W$ such that $V^{\prime} \cap V$ is nonsingular in $V$.

PROOF. If $\gamma_{n-1} \notin$ image $\left\{H_{n-1}\left(V-V \cap V^{\prime}\right) \rightarrow H_{n-1}(V)\right\}$, then $0 \neq$ $\gamma_{n-1} \cap V^{\prime}=\gamma_{n-3} \in H_{n-3}\left(V \cap V^{\prime}\right)$ since $V \cap V^{\prime}$ is nonsingular. But $V$ ample in $W \Rightarrow V \cap V^{\prime}$ obeys the Lefschetz theorem in $V^{\prime}$, i.e., $\gamma_{n-3} \neq 0$ in $V^{\prime}$ since $n-3<\operatorname{dim}_{\mathrm{C}} V \cap V^{\prime}$. But $i_{*} \gamma_{n-1}=0$ in $W$, hence $\left(i_{*} \gamma_{n-1}\right) \cap V^{\prime}=0$ in $V^{\prime}$, but $\gamma_{n-3}$ is a representative of their intersection, contradiction. Q.E.D.

\subsection{CoROllaRY. $i_{*} \gamma_{n-1}=0$ implies}

$$
\gamma_{n-1} \in \operatorname{Im}\left\{H_{p-1}\left(V-\bigcup_{i}\left(V_{i} \cap V\right)\right) \rightarrow H_{p-1}(V)\right\}
$$

for $V_{i}$ nonsingular in $W$ and $\left\{V_{i} \cap V\right\}_{i}$ having normal crossings in $V$.

Proof. If $\gamma_{n-1}$ misses $V_{i}$ and $V_{j}$, then $\gamma_{n-1}$ misses $V_{i} \cap V_{j}$ and hence $V_{i} \cup V_{j}$. Q.E.D.

So if $V$ is ample in $W$, let $\left(W^{\prime}, V^{\prime}, \pi\right)$ be a resolution to normal crossings with $V^{\prime}=\bigcup_{i=0}^{m} V_{i}$. Let $V_{0}$ be the proper transformation, i.e., $\pi\left(V_{0}\right)=V$ and $\pi\left(V_{i}\right) \subset S$, singular locus of $V$, for $i>0$.

So Corollary 3.5 gives indications it might be true if say $V_{0}$ were ample. However, $V_{0}$ is in general not ample. If one has enough conditions on the intersection of $V_{i} \cap V_{j}$, e.g., $V_{i}-V_{i} \cap V_{0}$ is Stein for all $i$, or if the injection of $V_{0} \cap V_{i}$ into $V_{i}$ induces an injection on homology for dimensions up to $n-2$, then one can prove the result. The difficulty is that one cannot guarantee these extra conditions in general for arbitrary resolutions.

Also, from purely topological techniques, we can show if $V$ has isolated 
singularities without any other conditions on $V$, then $R_{p}(V)$ is generated by $\gamma_{p-q} \in H_{p-q}\left(M_{q}\right)$ (see Corollary 4.15 below). There are also strong indications that if singular locus of $V$ is a curve, then one can prove the result for ample $V$. Moreover, Corollary 5.12 (below) says that if $\omega$ is a $C^{\infty}(p, 0)$ form with a pole of order one on $V$, i.e., if $f$ is a local defining equation of $V$, then $f \omega$ is smooth, then the dual cycle to $\omega$ is of the desired type. As we have mentioned above, $W-V$ is generated by meromorphic $p$-forms with poles of order $k$ on $V$. But in general, we need $k>1$, and when one reduces $\omega$, a pole of order $k$, to a form with pole of order one (i.e., $\omega=\Sigma_{q=1}^{k} \omega_{q}$ where $\omega_{q}$ is a $(p-q-1, q-1)$ form with a pole of order one (see beginning of $\S 5$ for definitions), then the technique of proof breaks down in the general case. So we have

3.6 Question. For $V$ ample in $W$ and $V^{\prime}$ a resolution to normal crossings, is $R_{p}\left(V^{\prime}\right)$ generated by $\gamma_{p-q} \in H_{p-q}\left(M_{q}\right)$ ?

4. Residues in the general case. In the previous section we have classified the residues in case the hypersurface has normal crossings, which is the case when one resolves singularities. However, one cannot in general resolve singularities for arbitrary complex manifolds, and even if one could, for technical reasons it might not be the best approach to the problem at hand. As a result, we now will look at the residues of arbitrary hypersurfaces without resolving singularities. The reason we did $\S 2$ is that, first of all, it will be necessary to consider the normal crossings in great detail in [5], and secondly, it provides a good example to have in mind in this section.

In this section $W$ will be an arbitrary $n$-complex dim manifold and $V$ an arbitrary subvariety.

4.1 Definition. A stratification of $V$ is the expression of $V$ as the disjoint union of a locally finite set of complex manifolds, call the strata, such that the boundary of each stratum is the union of a set of lower-dimensional strata. (See Whitney [27, esp. pp. 227-230], for a discussion of stratifications.)

From the definition it easily follows that the closure of each stratum is an analytic variety of the same dimension of the stratum and the boundary of a stratum is an analytic variety of lower dimension.

4.2 Definition. The stratification $\left\{M_{i}^{\prime}\right\}$ of $V$ is a refinement of the stratification $\left\{M_{i}\right\}$ of $V$ if for each stratum $M_{i}^{\prime}$, either $M_{i}^{\prime}$ is a stratum of $\left\{M_{i}\right\}$ or $M_{i}^{\prime} \subset$ $M_{j}$ such that $\partial M_{i}^{\prime} \cap \bar{M}_{j} \subseteq \partial M_{i}^{\prime}$ as subsets of the stratification.

Note. $\partial M_{i}^{\prime} \cap \bar{M}_{j}$ makes sense as a subset of $\left\{M_{i}^{\prime}\right\}$ since each stratum of $\partial M_{i}^{\prime}$ is either a stratum of $\left\{M_{i}\right\}$ or is contained in some stratum, which must either be a stratum of the boundary of $M_{j}$ or $M_{j}$ itself.

\subsection{Lemma. Any two stratifications of $V$ have a common refinement.}


Proof. Let $\left\{M_{i}\right\}$ and $\left\{M_{j}^{\prime}\right\}$ be two stratifications of $V$. Then $M_{i} \cap M_{j}^{\prime}$ is a complex subvariety of $V$, since each $M_{i}$ is locally given by the zeros of analytic functions. Thus we can stratify $M_{i} \cap M_{j}^{\prime}$ by taking successive nonsingular points, i.e., for any variety $V$ with singular locus $S$, $\operatorname{dim}_{\mathrm{C}} S<\operatorname{dim}_{\mathrm{C}} V$, so that this process terminates in stratification. Then let $\left\{M_{i}^{\prime \prime}\right\}$ be the stratification given by the stratification of $M_{i} \cap M_{j}^{\prime}$.

It is clear that $\partial\left(M_{i} \cap M_{j}^{\prime}\right) \cap \bar{M}_{i} \subseteq \partial\left(M_{1} \cap M_{j}^{\prime}\right)$. Q.E.D.

For each stratum $M_{i}$, say $\operatorname{dim}_{\mathrm{C}} M_{i}=d$, let $T_{0}\left(M_{i}, P\right)$, for $P \in M_{i}$, denote the $(2 d-1)$ direction of its tangent space, i.e., $T_{0}\left(M_{i}, P\right) \in G(2 n-1,2 d-1)$, where $G(2 n-1,2 d-1)$ is the Grassmannian of $(2 d-1)$-planes in $(2 n-1)$ space. Then take all pairs $\left(P, T_{0}\left(M_{i}, P\right)\right) \in W \times G(2 n-1,2 d-1)$ and let $\tau\left(M_{i}\right)$ be the closure of this set in $W \times G(2 n-1,2 d-1)$. Then for $P \in \bar{M}_{i}$, we define $\tau\left(M_{i}, P\right)=\left\{\alpha \mid(P, \alpha) \in \tau\left(M_{i}\right)\right\}$. For $P \in M_{i}, \tau\left(M_{i}, P\right)=T_{0}\left(M_{i}, P\right)$.

4.4 Definition. We say $\left\{M_{i}\right\}$ is a Whitney stratification $A$ of $V$ if $P \in M_{i}$ $\subset \bar{M}_{j}$ and $T\left(M_{i}, P\right)$ is the tangent plane of $P$ in $M_{i}$, then $T\left(M_{i}, P\right) \subset T$ for all $T \in T\left(M_{j}, P\right)$, i.e., if $v \in T\left(M_{i}, P\right)$, then $\exists P_{m} \in M_{j}$ and $v_{m} \in T\left(M_{j}, P_{m}\right)$ such that $v_{m} \rightarrow v$.

Whitney [27] has shown

\subsection{THEOREM. Any stratification has a refinement to a Whitney stratifica-} tion $A$.

As a corollary we have

4.6 Corollary. $V \subset W$ may be stratified so that the following is true: If $P_{0} \in M$, a stratum of dimension $d$, and if $M_{0}$ is a neighborhood of $P_{0}$ in $M$ such that through each point $P \in M_{0}$ there is a $2(n-d)$-dimensional plane $T(P)$ transverse to $M$ at $P$ with the $T(P)$ continuous in $P$ and disjoint, then there exists a neighborhood $U$ of $P_{0}$ in $W$ such that for all $P \in U \cap M_{0}, T(P)$ is transverse to $M^{\prime} \cap U$ for all strata $M^{\prime}$.

Proof. Since this is a local result, we consider $M_{0}$ contained in some coordinate chart in $W$ and then a plane means a plane in $\mathbf{C}^{n}$.

Suppose there does not exist such a neighborhood $U$. Then there exists $P_{m}$ $\in M_{0}$ such that $T\left(P_{m}\right)$ meets some stratum $M^{\prime}$ nontransversely at $Q_{m}$ and $Q_{m} \rightarrow$ $P_{0}$ as $m \rightarrow \infty$.

Since the stratification is locally finite, we can assume all the $Q_{m}$ belong to the same strata $M^{\prime}$. Then we have at $Q_{m}, \operatorname{dim}\left(T\left(P_{m}\right)+T\left(M^{\prime}, Q_{m}\right)\right)<2 n$. But as $m \rightarrow \infty ; T\left(P_{m}\right) \rightarrow T\left(P_{0}\right)$ since $T\left(P_{m}\right)$ are continuous in $P_{m} ;$ also $Q_{m} \rightarrow P_{0}$ which implies by passing to a subsequence if necessary, that we have $T\left(M^{\prime}, Q_{m}\right)$ $\rightarrow T \in T\left(M^{\prime}, P_{0}\right)$. By the choice of the stratification of Theorem 4.5, $T\left(M, P_{0}\right)$ 
$\subset T$. However, by continuity at $P_{0}, \operatorname{dim}\left(T\left(P_{0}\right)+T\right)<2 n$, contradiction of the fact that $T\left(P_{0}\right)$ meets $M$ transversely at $P_{0}$. Q.E.D.

Let $M \subset \bar{M}^{\prime}$ where $M$ and $M^{\prime}$ are strata. By using the corollary we construct the tubular neighborhood of $M$ in $M^{\prime}$ :

Let $M$ be a stratum of complex dimension $d$. Then let $T(M)$ be a tubular neighborhoood of $M$ in $W$ and $\pi: T(M) \rightarrow M$ be the projection. For $P \in M$, $\pi^{-1}(P)$ is a $2(n-d)$-dim ball transverse to $M$ at $P$ and $\pi^{-1}(P)$ depend continuously on $P$ and they are disjoint. Therefore there exists $U$, a neighborhood of $P$ in $W$, such that $\forall Q \in U \cap M, \pi^{-1}(Q)$ meets $U \cap M^{\prime}$ transversely. Then shrink the fibres of $T(M)$ such that $\forall Q \in U \cap M, \pi^{-1}(Q) \subset U$ and $\pi^{-1}(Q)$ is a $2(n-d)$-dim disk.

Cover $M$ by a locally finite cover $\left\{U_{\alpha}\right\}$ of such sets. Then patch together the new fibres of $T(M)$ via partition of unity to get a new tubular neighborhood of $M$ in $W$ such that, if $\pi: T(M) \rightarrow M$ is the projection, then for $Q \in M, \pi^{-1}(Q)$ is a $2(n-d)$-dim ball which intersects $M^{\prime}$ transversely, for all $M^{\prime}$.

We pick the fibres of each stratum such that they respect the fibres of the previous strata in the following sense:

Suppose $M_{d}$ is one of the lowest-dimensional strata and its complex dimension is $d$. We form $\pi: T\left(M_{d}\right) \rightarrow M_{d}$ with all $P \in M_{d}, \pi^{-1}(P)$ is a $2(n-d)$ dim ball and if $M_{d} \subset M_{d+j}\left(\operatorname{dim}_{\mathrm{C}} M_{d+j}=d+j\right)$, then $\pi^{-1}(P) \cap M_{d+j}$ is a $2 j$-dim chain. Furthermore $\partial\left(\pi^{-1}(P)\right) \cap M_{d+j}$ is a transverse intersection of a $2(d+j)$-dimension manifold with an arbitrary small $2(n-d)-1$-dimension sphere, say $\tau(P)$, in a $2 n$-dimensional space, i.e., $2 d+2 j+2 n-2 d-1-2 n=$ $2 j-1$-dim cycle in $M_{d+j}$. As $P$ varies in a $C^{\infty}$ fashion in $M_{d}$, the $2 n-2 d-1$. dimensional sphere varies $C^{\infty}$ with each sphere disjoint.

In fact $\partial\left(\pi^{-1}(P)\right) \cap M_{d+j}$ is a $(2 j-1)$-dimensional $C^{\infty}$ manifold, since the intersection is transverse. Thus, $\pi^{-1}(P) \cap M_{d+j}$ is a cone over a $(2 j-1)$-dimensional manifold. So, for $P \in M_{d}$, a neighborhood $U$ of $P$ in $M_{d}$ can be chosen so that $\pi^{-1}(U) \cap M_{d+j}$ is the product of $U \times$ (cone cver a manifold) and each of the fibres will be homeomorphic. We have a $2(n-d-j)$ normal direction in $\tau(P)$ to $M_{d+j} \cap \tau(P)$ and we pick this direction for the normal bundle of $M_{d+j}$ in $W$ at $M_{d+j} \cap \tau(P)$. Since $\tau(P)$ varies in a $C^{\infty}$ way with $P$, we can pick these directions in a $C^{\infty}$ way. Then extend this to all of $\widetilde{M}_{d+j}=M_{d+j}-$ int $T\left(M_{d}\right)$, where int $T\left(M_{d}\right)=$ interior in $W$ of $T\left(M_{d}\right)$. Thus, we have constructed $\pi$ : $T\left(\tilde{M}_{d+j}\right) \rightarrow \widetilde{M}_{d+j}$.

For all $P \in \widetilde{M}_{d+j} \cap \tau\left(M_{d}\right)$, where $\tau\left(M_{d}\right)=\partial\left(T\left(M_{d}\right)\right)$, we have that $\pi^{-1}(P)$ $\subset \tau\left(M_{d}\right)$ and since $T\left(M_{d}\right)$ meets all the strata transversely, we can make sure that $T\left(\tilde{M}_{d+j}\right)$ also has this property.

Now, suppose all $\pi: T\left(\tilde{M}_{k}\right) \rightarrow \widetilde{M}_{k}$ have been constructed for all dimensions 
less than $d$. Then for $P \in \widetilde{M}_{d-k} \subset M_{d}$ we have $\pi^{-1}(P)=2(n-d-k)$-dimensional ball which meets $M_{d}$ transversely and $\left[\tau(P)=\left(\partial \pi^{-1}(P)\right)\right] \cap M_{d}$ is the transverse intersection of a $2(n-d+k)-1$ sphere with a $2 d$-dim manifold. We have chosen the smallest $k$ possible. There is a $2(n-d)$ normal direction in $\tau(P)$ to this intersection which varies in a $C^{\infty}$ fashion with $P$ until $P$ meets $\partial \widetilde{M}_{d-k}$ and then we have $P \in \tau\left(\tilde{M}_{d-k-j}\right) \cap M_{d-k}$. We then move to $\pi^{-1}(Q)=$ $2(n-d+k+j)$-dim ball for $Q \in \widetilde{M}_{d-k-j}$. But since $P \in \tau\left(\widetilde{M}_{d-k-j}\right)$, we choose the fibre of $T\left(\widetilde{M}_{d-k}\right)$ to lie in $\tau(Q)=\partial \pi^{-1}(Q)$, a $2(n-d+k+j)-1$-dim sphere; we can continue in a $C^{\infty}$ fashion, i.e., $\tau(Q)$ intersects $M_{d}$ in a $(2 k+2 j-1)$-dim submanifold and hence the intersection has a $2(n-d)$-dim normal direction in $\tau(Q)$ and the point $P \in \partial \widetilde{M}_{d-k}$ also lies in $\tau(Q)$ and we already chose a $2(n-d)$ dim normal direction near $P$, so we just continue along $M_{d} \cap \tau(Q)$.

We then extend this to all of $\widetilde{M}_{d}=M_{d}-\bigcup_{k \geqslant 1}$ int $T\left(\widetilde{M}_{d-k}\right)$ in a $C^{\infty}$ fashion yielding a normal bundle $\pi: T\left(\tilde{M}_{d}\right) \rightarrow \widetilde{M}_{d}$ such that each fibre meets each stratum transversely.

Thus we have constructed a normal bundle of each $\widetilde{M}_{d}$ such that the fibres respect those of $\widetilde{M}_{d-j} \subset \bar{M}_{d}$.

Let us introduce some notation: If $T\left(M_{j}\right)$ is the normal disk bundle of $M_{j}$ in $W$, let $\tau\left(M_{j}\right)=(\partial T)\left(M_{j}\right)$ denote the induced normal sphere bundle, i.e., fibres will be $(2 n-2 j-1)$-dimensional spheres. Then denote by $T\left(\bar{M}_{d}\right)$ the "bundle" we have just constructed over $\bar{M}_{d}$ and let $\tau\left(\bar{M}_{d}\right)=\partial\left(T \bar{M}_{d}\right)$.

Then we can perform the above construction in such a way that $\tau\left(\bar{M}_{d}\right)$ is a smooth $(2 n-1)$-dim submanifold of $W$ and also that $\partial \widetilde{M}_{d}$ is a smooth $(2 d-1)$ dim submanifold of $M_{d}$.

First of all, $\tau\left(\bar{M}_{d}\right)$ is a PL-manifold (i.e., underlying space is a topological manifold, but the induced differential structure has corners). To see this note that $T\left(\widetilde{M}_{d}\right)$ has as boundary $\tau\left(\tilde{M}_{d}\right)+T\left(\partial \tilde{M}_{d}\right)$ and $T\left(\partial \tilde{M}_{d}\right) \subset T\left(\tilde{M}_{d-j}\right)$ as a submanifold. What we have done is glued $T\left(\tilde{M}_{d}\right)$ to $T\left(\tilde{M}_{d-j}\right)$ along $T\left(\partial \tilde{M}_{d}\right)$ (this is essentially what the topologist refer to as plumbing; see Figure 1 where the shaded region is $T\left(\partial \tilde{M}_{d}\right)$. This will have a corner along $\tau\left(\partial \tilde{M}_{d}\right)$. However by standard arguments involving plumbing, see e.g. Milnor [16], we can smooth these corners so that this is a differentiable manifold.

To see this smoothing intuitively, suppose we have $P \in M_{0}$, i.e., a zero-dim strata and then $S_{P}^{2 n-1} \cap M_{d}$ is a $(2 d-1)$-dimensional submanifold. Since this is purely a local problem (i.e., if we can smooth in a small neighborhood, then we can patch together via a partition of unity to get a global smoothing), choose a small neighborhood $R^{2 d-1}$ of $S_{P}^{2 n-1} \cap M_{d}$. Then we have that $\partial R_{+}^{2 d}=R^{2 d-1}$ where $R_{+}^{2 d}\left(\subset M_{d}\right)$ is the half-plane of $R^{2 d}$, i.e., $S_{P}^{2 n-1} \cap M_{d}$ is the boundary of $\widetilde{M}_{d}$, submanifold of $M_{d}$. Let $D^{2 n-2 d}$ be the normal plane to $M_{d}$ (i.e., $R_{+}^{2 d} \times$ 
$D^{2 n-2 d}$ is the normal bundle) such that $R^{2 d-1} \times D^{2 n-2 d} \subset S_{P}^{2 n-1}$. Then

$$
\partial\left(R_{+}^{2 d} \times D^{2 n-2 d}\right)=R^{2 d-1} \times D^{2 n-2 d}+R_{+}^{2 d} \times S^{2 n-2 d-1}
$$

and

$$
\partial\left(R^{2 d-1} \times D^{2 n-2 d}\right)=-R^{2 d-1} \times S^{2 n-2 d-1} .
$$

So on $S_{P}^{2 n-1}$ we cut out $R^{2 n-1} \times D^{2 n-2 d}$ and attach $R_{+}^{2 d} \times D^{2 n-2 d}$ along its boundary. This has a corner at $R^{2 d-1} \times S^{2 n-2 d-1}$, i.e., if $Q \in R^{2 d-1} \times$ $S^{2 n-2 d-1}$, then a neighborhood of $Q$ in this $(2 n-1)$-dimensional PL-manifold is of the form $\left(R^{2 d-1} \times S^{2 n-2 d-1}\right) \times\left(R_{+} \cup R_{+}\right)$where $R_{+} \cup R_{+} \subset R \times R=$ normal plane of $R^{2 d-1} \times S^{2 n-2 d-1}$ in $W$. Then we just smooth out this corner in the normal plane.

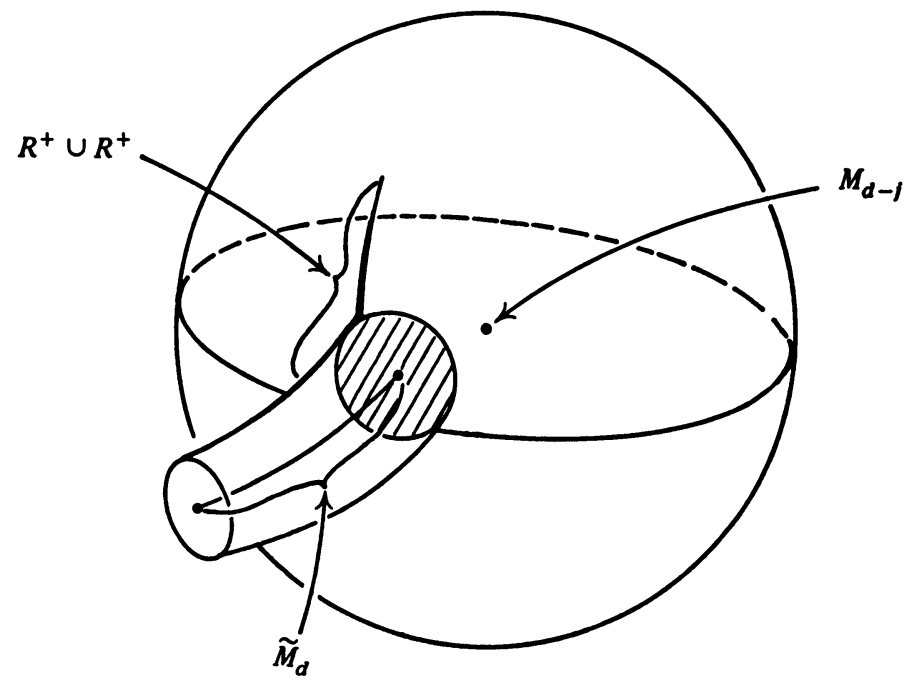

Figure 1

4.7 LEMMA. $T\left(\bar{M}_{d}\right)$ is the cone over $\tau\left(\bar{M}_{d}\right)$ with vertex $\bar{M}_{d}$, i.e., $\bar{M}_{d}$ is a strong deformation retract of $T\left(\bar{M}_{d}\right)$ and if $\left\{f_{t}\right\}$ gives the deformation (with $f_{0}$ the identity), then $f_{t} \mid \tau\left(\bar{M}_{d}\right)$ is a diffeomorphism for $t<1$ and $f_{t}\left(\tau\left(\bar{M}_{d}\right)\right) \cap$ $f_{t^{\prime}}\left(\tau\left(\bar{M}_{d}\right)\right)=\varnothing$ for $t \neq t^{\prime}$. Furthermore for any stratum $M_{d+j}$ with $M_{d} \subset \bar{M}_{d+j}$, $f_{t} \mid M_{d+j} \cap T\left(\bar{M}_{d}\right) \subset M_{d+j}$ for all $t$. For $Q \in M_{j}, f_{1}^{-1}(Q)$ is a $2(n-2 j)$-dimensional chain.

Proof. The deformation is given by shrinking along the fibres of the normal bundles of $\tilde{M}_{d+k}$. The plumbing causes no difficulty because we are glueing together along normal bundles. 
To see that $f_{1}^{-1}(Q)$ is a $(2 n-2 j)$-dim chain $Q \in M_{j}$, let $M_{k}$ be the lowestdimensional stratum of $\bar{M}_{d}$. Then it is clear that for $Q \in M_{k}$ that $f_{1}^{-1}(Q)$ is a $2(n-k)$-dim ball since $T\left(\bar{M}_{d}\right) \mid M_{k}=T\left(M_{k}\right), M_{k}$ being a complex $k$-dim manifold without boundary.

Suppose $Q \in M_{k+j} \cap T\left(M_{k}\right)$. Choose a Riemann metric in $T\left(M_{d}\right)$ so that the fibres are balls of radius one. Then suppose $Q \in \pi^{-1}(P), P \in M_{k}$, and $Q$ is of distance $t, 0<t \leqslant 1$, from $P$. Choose a collared neighborhood $\partial \widetilde{M}_{k+j} \times I$ of $\partial \tilde{M}_{k+j}$ in $\widetilde{M}_{k+j}$. Let $Q^{\prime} \in \tau\left(\bar{M}_{k}\right) \cap M_{k+j} \subset \partial \widetilde{M}_{k+j}$ be that unique point which passes through the given $Q$ when we shrink $T\left(\bar{M}_{k}\right)$ along its fibres via the Riemannian metric. Then the collapsing of $T\left(\widetilde{M}_{k+j}\right) \mid\left(\partial \widetilde{M}_{k+j} \times I\right)$ will send the fibre over $\left(Q^{\prime}, 2 t\right) \in \partial \widetilde{M}_{k+j} \times I$ onto $Q$ and will send the fibre over $\left(Q^{\prime}, t\right)$ for $1 / 2 \leqslant$ $t \leqslant 1$ onto $\left(Q^{\prime}, 2 t-1\right)$. The collapsing of $T\left(\tilde{M}_{k+j}\right) \mid\left(\tilde{M}_{k+j}-\left(\partial \widetilde{M}_{k+j} \times I\right)\right)$ will be along the fibres via a Riemannanian metric in $T\left(\widetilde{M}_{k+j}\right)$. Q.E.D.

Of course the construction of $T\left(\bar{M}_{d}\right)$ and the deformation retract depend on the choice of various normal bundles of the $M_{j}$. However, we have

4.8 LEMMA. $T\left(\bar{M}_{d}\right)$ is isotopic to $T^{\prime}\left(\bar{M}_{d}\right)$ for any other choice of normal bundles $T^{\prime}\left(\tilde{M}_{j}\right)$. Furthermore, if $\left\{g_{t}\right\}$ is the isotopy, then $g_{1} \mid f_{1}^{-1}(Q)=$ $\left(f_{1}^{\prime}\right)^{-1}(Q)$ where $f_{1}, f_{1}^{\prime}$ are from Lemma 4.7 and $g_{t}\left(\bar{M}_{d}\right)=\bar{M}_{d}$.

Note. Recall two submanifolds $M_{1}, M_{2} \subset W$ are isotopic if there is a smooth map. $G: M_{1} \times I \rightarrow W$ such that $g_{t}=G(\cdot, t)$ is a diffeomorphism for all $t$ and $g_{0}$ is the identity map, $g_{1}\left(M_{1}\right)=M_{2}$.

Proof. The isotopy follows from the uniqueness (up to isotopy) of normal bundles, e.g. if $M_{k}$ is the smallest dimensional stratum in $M_{d}$, then $T\left(M_{k}\right)$ is isotopic via $\left\{g_{t}\right\}$ to $T^{\prime}\left(M_{k}\right)$. Let $M_{k+j}$ be the smallest stratum $M_{d}$ which has $M_{k}$ as part of its boundary and set

$$
\tilde{M}_{k+j}=M_{k+j}-T\left(M_{k}\right), \quad \tilde{M}_{k+j}^{\prime}=M_{k+j}-T^{\prime}\left(M_{k}\right) .
$$

If we take $T\left(\partial \tilde{M}_{k+j}\right)$ in $\tau^{\prime}\left(M_{k}\right)$, then $g_{1} \mid T\left(\partial \tilde{M}_{k+j}\right)$ is a normal bundle to $\partial \tilde{M}_{k+j}^{\prime}$ in $\tau^{\prime}\left(M_{k}\right)$, hence is isotopic via $\left\{h_{t}\right\}$ to $T^{\prime}\left(\partial \widetilde{M}_{k+j}^{\prime}\right)$ in $\tau^{\prime}\left(M_{k}\right)$.

But $\left\{g_{t}\right\}$ extends to an isotopy between $\widetilde{M}_{k+j}$ and $\widetilde{M}_{k+j}^{\prime}$, because we have an isotopy between two boundaries of two manifolds which are diffeomorphic, so by taking a collared neighborhood $\left(\partial \widetilde{M}_{k+j}\right) \times I$ of the boundaries we can extend the isotopies such that $g_{t}\left|\partial \widetilde{M}_{k+j} \times\{1\}=g_{0}\right| \partial \tilde{M}_{k+j} \times\{1\}=\partial \tilde{M}_{k+j}^{\prime} \times$ $\{1\}$ and extend to the rest of the manifold by the diffeomorphism $g_{0}$. Hence we can extend $\left\{g_{t}\right\}$ to an isotopy of $T\left(\tilde{M}_{k+j}\right)$ such that $g_{1} \mid T\left(\tilde{M}_{k+j}\right)$ is a normal bundle to $\widetilde{M}_{k+j}^{\prime}$. Then $g_{1} \mid T\left(\widetilde{M}_{k+j}\right)$ is isotopic via $\left\{h_{t}\right\}$ to $T^{\prime}\left(M_{k+j}\right\}$ in such a way that it extends the above $h_{t} \mid g_{1} T\left(\partial \tilde{M}_{k+j}\right)$, because if two normal bundles 
have the same fibre, then the isotopy can be chosen so that on this fibre it is the identity map at each $t$.

Then we continue in this fashion to get the isotopy between $T\left(M_{d}\right)$ and $T^{\prime}\left(M_{d}\right)$. But $g_{t} \mid \bar{M}_{d}$ is not the identity, only a diffeomorphism for all $t$. Actually, it is more: $g_{t} \mid \bar{M}_{k+j}$ is an isotopy on each stratum. Furthermore, from the above construction it is clear that $g_{1} \mid f_{1}^{-1}(Q)=\left(f_{1}^{\prime}\right)^{-1}(Q)$ for all $Q$. Q.E.D.

Let $V$ be any variety in a complex manifold $W$, and $\left\{M_{i}\right\}$ a Whitney stratification $A$ of $V$. Then we can construct the tubular neighborhoods of $M_{i}$ in $W$ and denote it by $T\left(V ; M_{i}\right)$, which we call the tubular neighborhood of $V$ in $W$ relative to $\left\{M_{i}\right\}$. If $\pi: T\left(V ; M_{i}\right) \rightarrow V$ is the retraction of Lemma 4.7, then $\pi^{-1}(Q)$ is the fibre over $Q$.

4.9 THEOREM. Let $V$ be a subvariety of $W$ and $\left\{M_{i}\right\}$ and $\left\{M_{1}^{\prime}\right\}$ Whitney stratifications $A$ of $V$. Then $T\left(V ; M_{i}\right)$ is isotopic via $\left\{g_{t}\right\}$ to $T\left(V ; M_{1}^{\prime}\right)$ and $g_{t}(V)=V$ for all $t$.

Proof. It suffices to prove 4.9 for $\left\{M_{i}^{\prime}\right\}$ a refinement of $\left\{M_{i}\right\}$ because of Lemma 4.3 and Theorem 4.5. But once we have a refinement, the result will follow from the tubular neighborhood theorem for manifolds with boundary: if $N$ is a submanifold of $W$ such that $\partial N \cap M \subset \partial M$ with $N$ transverse to $\partial M$ or if $\partial N \subset M-\partial M$, then $N$ has a tubular neighborhood in $M$ which is unique up to isotopy (see Milnor [15, p. 57]). So the same construction of the isotopy in Lemma 4.8 will go through with the obvious modifications, because any stratum $M_{i}^{\prime} \subset M_{i}$, then either $\partial M_{i}^{\prime} \subset M_{i}$ or if $\partial M_{i}^{\prime}$ contains a stratum $M_{j}^{\prime}$ which is not a stratum of $\partial M_{i}$ then $\bar{M}_{i}^{\prime}$ meets $\partial M_{i}$ transversely at $M_{j}^{\prime}$. Q.E.D.

Let $S$ be any subvariety of a variety $V$ in some manifold $W$. Then let $\left\{M_{i}\right\}$ be a Whitney stratification $A$ of $V$ in $W$ relative to $S$, i.e., $M_{i} \cap S \neq \varnothing$ implies $M_{i} \subset S$. Such a stratification clearly exists and will also be a Whitney stratification $A$ of $S$ in $W$. Then we can form $T\left(S ; M_{i}\right)$ and put $T\left(S ; M_{i}\right) \cap V=T\left(S, V ; M_{i}\right)$ which will be called the tubular neighborhood of $S$ in $V$ relative to $M_{i}$. Thus we have shown

4.10 Corollary. Let $\left\{M_{i}\right\}$ and $\left\{M_{i}^{\prime}\right\}$ be Whitney stratifications $A$ of $V$ in $W$ relative to $S$. Then $T\left(S, V ; M_{i}\right)$ is homotopic to $T\left(S, V ; M_{i}^{\prime}\right)$ via a family of homeomorphisms which will come from the restrictions of diffeomorphisms. If both $\tau\left(S, V ; M_{i}\right)$ are contained in the singular part of $V$, then they will be isotopic.

4.11 Definition. Let $S$ be a subvariety of a variety $V \subset W$, a manifold. Then $T(S, V)$ will be called the tubular neighborhood of $S$ in $V$ (relative to $W$ ), where $T(S, V)$ is constructed for any stratification of $V$ relative to $S$. If $\gamma_{p} \in$ $H_{p}(\tau(S, V))$, where $\tau(S, V)=\partial T(S, V)=(\partial T(S)) \cap V=\tau(S) \cap V$, then $\gamma_{p}$ will be called a tube over a cycle in $S$. 
4.12 THEOREM. Let $W$ be an $n$-complex dimensional manifold and $V a$ subvariety of codimension q, i.e. $\operatorname{dim}_{\mathrm{C}} V=n-q$. Then $\operatorname{ker}\left\{H_{p}(W-V) \rightarrow H_{p}(W)\right\}$ $=$ geometric $p$-residues of $V=R_{p}(V)$ is generated by tubes over cycles in $V$.

Proof. Let $\gamma_{p} \in H_{p}(W-V)$ and $\gamma_{p}=\partial c_{p+1}$. Choose a Whitney stratification $A$ of $V$ in $W$ and construct $T(V, W)$. Then since $M_{i}$ are manifolds and we are working in a manifold $W$, we can choose $c_{p+1}$ such that it intersects each stratum of $V$ transversely. That is $c_{p+1}$ respects the fibres of $V$, i.e., if $\gamma_{p-2 q+1}=$ $c_{p+1} \cap V$ (i.e. $V$ is a codimension $q$, hence has a dual cohomology class of dimension $2 q$ and the cap product of $c_{p+1}$ with $V$ will then define a $p-2 q+1$-dim chain on $V$, but $\partial c_{p+1} \cap V=\varnothing$, so that this $p-2 q+1$-dim chain, $\gamma_{p-2 q+1}$, is actually a cycle on $V$ ), then $T \gamma_{p-2 q+1} \subset c_{p+1}$, where $T \gamma_{p-2 q+1}=c_{p+1} \cap$ $T(V)$.

Thus $\partial\left(c_{p+1}-T \gamma_{p-2 q+1}\right)=\gamma_{p}-\tau\left(\gamma_{p-2 q+1}\right)$, where $\tau\left(\gamma_{p-2 q+1}\right)=$ $c_{p+1} \cap \tau(V)$. Q.E.D.

Note that a stratification of $V$ can be chosen so that the $M_{n-q}$ strata ( $\operatorname{dim}_{\mathrm{C}} M_{i}=i$ ) are the connected components of the nonsingular part of $V$. Also if $\tilde{\gamma}_{i}=\tilde{M}_{i} \cap c_{p+1}$ where $\tilde{M}_{i}=M_{i}-\Sigma_{j \geqslant 1} T\left(\tilde{M}_{i-j}\right)$ and $\tilde{\gamma}_{i} \in H_{p+1+2 i-2 n}\left(\tilde{M}_{i}, \partial \tilde{M}_{i}\right)$, then $\gamma_{p}=\sum_{i=[(2 n-p-1) / 2]}^{n-q} \tau\left(\tilde{\gamma}_{i}\right)$.

Note. The essential criterion to construct the tubular neighborhood in Theorem 4.12 was Theorem 4.5. Thus, if $V$ is any simplicial complex contained in a real smooth manifold $W$ such that $V$ has a Whitney stratification $A$, then Theorem 4.12 is also true for $V$ with respect to $W$. In particular, any locally finite polyhedron in $W$ will have a regular neighborhood which will satisfy the Whitney stratification $A$. Also any real subanalytic set $V$ will satisfy the Whitney stratification $A$. We say $V$ is subanalytic at $P$ if there exists an open set $U \ni P$ and proper real analytic maps $f_{i}: X_{i} \rightarrow W\left|U, g_{i}: Y_{i} \rightarrow W\right| U$ for $X_{i}, Y_{i}$ and $W$ real analytic spaces such that $V \cap U=\bigcup_{i}\left\{\operatorname{Im}\left(f_{i}\right)-\operatorname{Im}\left(g_{i}\right)\right\}$. Any real semianalytic set is subanalytic (Hironaka [10]).

Given Theorem 4.12, the obvious question is: What happens if $\gamma_{p-2 q+1} \sim$ 0 in $V$ ? I.e., Corollary 2.8 says that if $\gamma_{p-1} \sim 0$, then $0 \neq\left[\gamma_{p-2 q+1}\right] \in$ $H_{p-1}(\tau(S, V))$ where $S$ is the singular locus of $V$. So if $\gamma_{p-1} \sim 0$ in $V$, does this imply $0 \neq\left[\gamma_{p-2 q+1}\right] \in H_{p-2 q+1}(\tau(S, V))$ ? Instead of this, we have

4.13 COROLlaRY. $\left[\gamma_{p}\right] \in R_{p}(V)$ with $\gamma_{p}=\tau\left(\gamma_{p-2 q+1}\right)$ such that $\boldsymbol{\gamma}_{p-2 q+1} \sim 0$ in $V$, then $0 \neq\left[\gamma_{p-2 q+1}\right] \in H_{p-2 q+1}\left(\tau\left(S^{\prime}, V\right)\right)$ for $S^{\prime}$ some subvariety of $S$, the singular locus of $V$.

PROOF. If $\gamma_{p-2 q+1} \cap S=\not \varnothing$, i.e.,

$$
\gamma_{p-2 q+1} \in \text { image }\left\{H_{p-2 q+1}(V-\tau(S, V)) \rightarrow H_{p-2 q+1}(V)\right\}
$$


and $\gamma_{p-2 q+1}=\partial c_{p-2 q+2}$, then

$$
c_{p-2 q+2} \cap \tau(S, V)=\gamma_{p-2 q+1}^{\prime} \in H_{p-2 q+1}(\tau(S, V))
$$

and $\tau\left(\gamma_{p-2 q+1}\right) \sim \tau\left(\gamma_{p-2 q+1}^{\prime}\right)$ in $W-V$.

Suppose $\gamma_{p-2 q+1} \cap S \neq \varnothing$, i.e., $\gamma_{p-2 q+1} \cap M_{i}=\gamma_{p+2 i+1-2 n} \in$

$H_{p+2 i+1-2 n}\left(M_{i}\right)$, then $\gamma_{p+2 i+1-2 n} \sim 0$ in $M_{i}$ with homology taken with closed support, i.e.,

$$
\gamma_{p+2 i+1-2 n}=\left(\partial c_{p-2 q+2}\right) \cap M_{i}=\partial\left(c_{p-2 q+2} \cap M_{i}\right) \pm c_{p-2 q+2} \cap \partial M_{i} .
$$

Let $M_{d}$ be the lowest-dimensional stratum with which $\gamma_{p-2 q+1}$ has nonzero intersection. Then if $c_{p-2 q+2} \cap \partial M_{d}=\varnothing$, we can construct $\tau\left(c_{p-2 q+1}\right) \subset W-V$ such that $\partial\left(\tau\left(c_{p-2 q+2}\right)\right)=-\tau\left(\gamma_{p-2 q+1}\right)$.

Thus suppose $c_{p-2 q+1} \cap \partial M_{d} \neq \varnothing$. Set $S^{\prime}=\partial M_{d}$, a subvariety of $S$, i.e. $S^{\prime}=\bar{M}_{d}-M_{d}$, and $\gamma_{p-2 q+1} \cap M_{d}$ will be a $(p+1+2 d-2 n)$-cycle of $\tau\left(S^{\prime}, M_{d}\right)$. If we set $\gamma_{p-2 q+1, T}=\gamma_{p-2 q+1} \cap T(S)$, then $\gamma_{p-2 q+1, T}$ is a $(p-2 q+1)$-dimensional chain of $\tau\left(S^{\prime}, V\right)$ because $\gamma_{p-2 q+1} \cap M_{d} \subset \tau\left(S^{\prime}, M_{d}\right)$ and $\gamma_{p-2 q+1, T}$ has transverse intersection with each stratum; hence in the construction of $\tau\left(S^{\prime}, V\right)$, the fibres can be chosen to respect the transverse intersection of $\gamma_{p-2 q+1, T}$

Let $\gamma_{p-2 q}=\gamma_{p-2 q+1, T} \cap \tau(S, V)$. Then

$$
\gamma_{p-2 q}=\left(\partial c_{p-2 q+2}\right) \cap \tau(S, V)=\partial\left(c_{p-2 q+2} \cap \tau(S, V)\right) .
$$

Also

$$
c_{p-2 q+2} \cap \tau(S, V)=c_{p-2 q+1, \tau} \subset \tau\left(S^{\prime}, V\right)
$$

because $c_{p-2 q+2} \cap \bar{M}_{d}$ is the cone over $\gamma_{p-2 q+1} \cap M_{d}$ in $\bar{M}_{d}$ relative to $\partial M_{d}$. Thus

$$
\gamma_{p-2 q+1}^{\prime}=\gamma_{p-2 q+1, T}-c_{p-2 q+1, \tau} \in H_{p-2 q+1}\left(\tau\left(S^{\prime}, V\right)\right) .
$$

Also note that $\tau\left(\gamma_{p-2 q+1}^{\prime}\right) \subset W-V$, since $\gamma_{p-2 q+1, T}=\gamma_{p+1} \cap T(S, V)$ by transverse intersection and $c_{p-2 q+1, \tau} \subset V-S$. Furthermore $\tau\left(\gamma_{p-2 q+1}^{\prime}\right) \sim$ $\tau\left(\gamma_{p-2 q+1}\right)$ in $W-V$ because

$$
\partial\left(c_{p-2 q+2}-c_{p-2 q+2} \cap T(S, W)\right)=\gamma_{p-2 q+1}-\gamma_{p-2 q+1, T}+c_{p-2 q+1, \tau} .
$$

Furthermore, $0 \neq \gamma_{p-2 q+1}^{\prime} \in H_{p-2 q+1}\left(\tau\left(S^{\prime}, V\right)\right)$. This is because if $\gamma_{p-2 q+1}^{\prime}=\partial c_{p-2 q+2}^{\prime}$ for $c_{p-2 q+2}^{\prime} \subset \tau\left(S^{\prime}, V\right)$, then $\tau\left(c_{p-2 q+2}^{\prime}\right) \subset W-V$, i.e., the only obstruction to constructing the tube over $c_{p-2 q+2}^{\prime}$ lies in $S^{\prime}$, as $\gamma_{p-2 q+1}^{\prime} \cap M_{d}=\left(\partial c_{p-2 q+2}^{\prime}\right) \cap M_{d}=\partial\left(c_{p-2 q+2}^{\prime} \cap M_{d}\right) \pm c_{p-2 q+2}^{\prime} \cap \partial M_{d}$, 
but $c_{p-2 q+2}^{\prime} \cap \partial M_{d}=c_{p-2 q+2}^{\prime} \cap S^{\prime}=\varnothing$. Thus we have $\partial\left(\tau\left(c_{p-2 q+2}^{\prime}\right)\right)=$ $-\tau\left(\gamma_{p-2 q+1}^{\prime}\right)$ in $W-V$. Q.E.D.

4.14 CoROllaRY. If $\operatorname{dim} S=0$, then $\gamma_{p}=\tau\left(\gamma_{p-2 q+1}\right)$ implies either $0 \neq\left[\gamma_{p-2 q+1}\right] \in H_{p-2 q+1}(V-\tau(S, V))$ or $0 \neq\left[\gamma_{p-2 q+1}\right] \in$ $H_{p-2 q+1}(\tau(S, V))$.

4.15 CoROllary. If the dimension of the singular locus of $V$ is zero and $\left(W^{\prime}, V^{\prime}, \pi\right)$ is a resolution of $V$ to normal crossings with $V_{0}$ being the proper transform, then $R_{p}\left(V^{\prime}\right)$ is generated by cycles of the form $\tau_{1}\left(\gamma_{p-1,0}\right)$ for $\gamma_{p-1,0}$ $\subset V_{0}-\bar{M}_{2}$.

Proof. Let $U_{i}$ be small neighborhoods in $W$ of the isolated points $P_{i}$ and set $U=\bigcup_{i} U_{i}$. Put $U^{\prime}=\pi^{-1}(U)$. Then $\pi \mid W^{\prime}-U^{\prime} \rightarrow W-U$ is a biholomorphic homeomorphism because when we resolve singularities, we never change anything outside $U$.

But $0 \rightarrow R_{p}\left(V^{\prime}\right) \stackrel{\pi_{*}}{\rightarrow} R_{p}(V)$ and $R_{p}(V)$ is generated by $\tau\left(\gamma_{p-2 q+1}\right)$ for $0 \neq\left[\gamma_{p-2 q+1}\right] \in H_{p-2 q+1}(V-V \cap U)$ or $\left[\gamma_{p-2 q+1}\right] \in H_{p-2 q+1}(\partial U)$ and $\partial U$ is unchanged. Q.E.D. for Corollary 4.15.

Note. Recall by Proposition 3.1, $R_{p}(V) \simeq R_{p}\left(V^{\prime}\right)$, so that if $V \subset W$ can be blown down to a hypersurface with isolated singularities, we have the results discussed in $\S 3$.

Note. Just because $\gamma_{p-1} \sim 0$ in $V$ does not imply $\gamma_{p-1} \sim 0$ in $V_{0}$, e.g., let $V=\left\{x^{3}+y^{3}+z^{3}=0\right\}$ in $\mathrm{CP}_{3}$ with homogeneous coordinates $[x, y$, $z, w] . V$ is the cone over the elliptic curve $C \subset \mathrm{CP}_{2}$. Let $\nu$ be the normal bundle of $C$ in $\mathbf{C} P_{2}$, and $\nu^{*}$ the projectification of $\nu$, i.e., replace each fibre $C^{\prime}$ by $\mathbf{C} P_{1}$ by adding a point at infinity. If $C_{\infty}$ is the curve we obtain at infinity in constructing $\nu^{*}$, then by collapsing $C_{\infty}$ to a point $P_{\infty}$ we get $V$. If we blow up $V$ at $P_{\infty}$, we get $V^{\prime}=V_{0} \cup V_{1}$ where $V_{0} \approx \nu^{*}$ and $V_{1}=\mathrm{CP}_{2}$ with $V_{0} \cap V_{1}=C_{\infty}$. (In $V_{0}, C_{\infty}=C-3 F, F$ being the fibre.) Also $\tau\left(P_{\infty}, V\right) \approx \tau\left(C_{\infty}, \nu^{*}\right)$ and $H_{1}\left(\tau\left(C_{\infty}, \nu^{*}\right)\right)=Z \oplus Z \oplus Z_{3}\left(Z_{3}\right.$ because $C_{\infty} \cdot C_{\infty}=-3$ in $\left.\nu^{*}\right)$. But the free generators of $H_{1}\left(\tau\left(C_{\infty}, \nu^{*}\right)\right)$ do not bound in $V_{0}=\nu^{*}$.

4.16 COROLlARY. $R_{2 n-1}(V)=0$ and if $\gamma_{2 n-2}=\tau\left(\gamma_{2 n-2 q-1}\right)$ with $\gamma_{2 n-2 q-1} \sim 0$, then $\left[\gamma_{2 n-2 q-1}\right] \in H_{2 n-2 q-1}(\tau(S, V))$ where $S$ is the singular locus.

Proof. $R_{2 n-1}(V)$ can have at most one cycle, $\tau(V)=\partial(W-T(V))$.

If $\gamma_{2 n-2}=\tau\left(\gamma_{2 n-2 q-1}\right)$ and $0 \neq\left[\gamma_{2 n-2 q-1}\right] \in H_{2 n-2 q-1}\left(\tau\left(S^{\prime}, V\right)\right)$, but $\operatorname{dim}_{R} \tau\left(S^{\prime}, V\right)=2 n-2 q-1$, hence if $b_{2 n-2 q-1}\left(\tau\left(S^{\prime}, V\right)\right)=1, b_{i}=i$ th Betti number, then $\gamma_{2 n-2 q-1}=k \tau\left(S^{\prime}, V\right)$ for $k \neq 0$ and $\tau_{V}\left(\gamma_{2 n-2 q-1}\right)=$ $\partial\left(k \tau\left(S^{\prime}\right)-k T_{V}\left(\tau\left(S^{\prime}, V\right)\right)\right)$. 
If $b_{2 n-2 q-1}\left(\tau\left(S^{\prime}, V\right)\right)>1$ and $S^{\prime}$ is a proper subvariety of $S$, then let $V_{1}, \ldots, V_{k}$ be the components of $V$ near $S^{\prime}$. If $\gamma_{2 n-2 q-1}$ misses one of the components, say $V_{1}$, then we are in the situation discussed in the proof of Corollary 2.8, i.e., $\gamma_{2 n-2 q-1} \cap S \subset V-V_{1}$ will be the cone over part of $S^{\prime} \subset V_{1}$, so that instead of taking the tube so that we get $\gamma_{2 n-2 q+1}$, we first take the tube in the $V_{1}$ direction to get a $\gamma_{2 n-2 q+1} \subset V_{1}-S$, i.e., $\gamma_{2 n-2 q+1}^{\prime} \in$ $H_{2 n-2 q+1}(\tau(S, V))$. If $\gamma_{2 n-2 q-1}$ intersects each component, then $\gamma_{2 n-2 q-1}=$ $\sum_{i=1}^{k} k_{i} \tau\left(S^{\prime}, V_{i}\right)$ and $k_{i} \neq 0$ for all $i$; hence $\tau\left(\gamma_{2 n-2 q-1}\right) \sim 0$. Q.E.D.

Note. If $\operatorname{dim}_{\mathrm{C}} S=1$, we have that if $\gamma_{p-2 q+1} \sim 0$ in $V$, then $\gamma_{p+1} \cap M_{1}$ $=p+1+2-2 n \geqslant 0$, i.e., $p \geqslant 2 n-3$. But for $p=2 n-3$, we can have $\gamma_{2 n-3-2 q-1} \in H_{2 n-3-2 q-1}\left(\tau\left(S^{\prime}, V\right)\right)$ for $S^{\prime}$ a proper subvariety of $S$, ie.. $S^{\prime}$ $=\left\{M_{0}\right\}$. E.g, if $n=3$, this says we have a $\gamma_{3} \in H_{3}(W-V), \gamma_{3}=\tau\left(\gamma_{2}\right)$ with $\gamma_{2} \subset \tau(P, V)$ where $P$ is a singular point of $S$ and $\tau(P, V) \cap S$ will be a real onedimensional submanifold such that $\gamma_{2} \cdot(\tau(P, V) \cap S)=m>0$ in $\tau(P, V)$, a real three-dimensional algebraic variety. By $\gamma_{2} \cdot(\tau(P, V) \cap S)=m>0$, we mean $\gamma_{2}$ intersects these circles transversely in isolated points. Further we can also assume $V$ is locally irreducible at $P$, i.e., $\tau(P, V)$ has its third Betti number equal to one, since if $V=\bigcup_{i} V_{i}$, then $\tau(P, V)=\Sigma_{i} \tau\left(P, V_{i}\right) / \Sigma \tau\left(P, V_{i} \cap V_{j}\right)$ with $\tau\left(P, V_{i} \cap V_{j}\right) \subset S$, singular curve. But $\gamma_{2}$ having transverse intersection with $\tau(P, V) \cap S$ implies that $\gamma_{2} \subset \tau\left(P, V_{i}\right)$ for some $i$. I do not know of an example where this occurs. The obvious choices, $z^{a}=x^{b} y^{c}$ with g.c.d. $(a, b, c)=1$ (iff $V$ is locally irreducible at $(0,0,0)=P$ ) have as singular locus $C_{x} \cup C_{y}$ if $b$, $c>1$. But in all these cases $H_{2}(\tau(P, V))=0$. One shows this by mapping $f$ : $D^{2} \times D^{2} \rightarrow V \cap B$ where $B$ is the ball in $C^{3}$ of radius one by, if $l=1 . c . m .(a, b, c)$, $f(u, v)=\left(u^{l / b}, v^{l / c},(u v)^{l / a}\right)$ which is onto if g.c.d $(a, b, c)=1$. Then set $(u, v) \sim(p, q)$ iff $f(u, v)=f(p, q)$ and $\tau(P, V)=V \cap \partial B=\partial\left(D^{2} \times D^{2} / \sim\right)$.

Now let us return to constructing the Gysin sequence for an arbitrary variety of codimension $q$ in $W$. Locally, $V=\bigcup V_{q^{\prime}, i}$ where $V_{q^{\prime}, i}$ is an irreducible component of codim $q^{\prime} \geqslant q$. Then let $V_{q^{\prime}}=\bigcup_{i} V_{q^{\prime}, i}$ which is a subvariety of $V_{q}$ of codim $q-q^{\prime}$. Then $V=\bigcup_{q^{\prime}} V_{q^{\prime}}$. Note that if $\gamma_{p} \subset W-V$ and $\gamma_{p}=\partial c_{p+1}$, then $c_{p+1} \cap V_{q^{\prime}}=\gamma_{p-2 q^{\prime}+1}$. So we let $Z_{p-2 q+1}(V)_{\Delta}$ be those $\left(p-2 q^{\prime}+1\right)$-cycles of $V_{q^{\prime}}$ for $q^{\prime} \geqslant q$ over which the tube can be constructed in $W-V$, i.e., we take a $\left(p-2 q^{\prime}+1\right)$-cycle of $V_{q^{\prime}}$ and (perhaps) plumb it together with a $(p-2 q+1)$-cycle of $V_{q}$ to get a $p$-cycle of $W-V$.

If $\gamma, \gamma^{\prime} \in Z_{p-2 q+1}(V)_{\Delta}$, then we shall say that $\gamma$ is equivalent to $\gamma^{\prime}$, denoted $\gamma \sim \gamma^{\prime}$, if $\gamma-\gamma^{\prime}=\partial c$ where $c=\Sigma_{q^{\prime}} c_{p-2 q^{\prime}+2}$ and $c_{p-2 q^{\prime}+2}$ is a $(p-$ $\left.2 q^{\prime}+2\right)$-chain of $V_{q^{\prime}}$ and we have $\tau(\gamma)-\tau\left(\gamma^{\prime}\right)=-\partial \tau(c)$ in $W-V$. Then define

$$
H_{p-2 q+1}(V)_{\Delta}=\left(Z_{p-2 q+1}(V)_{\Delta}\right) / \sim .
$$

We call $H_{p-2 q+1}(V)_{\Delta}$ the tubular cycles. 
4.17 COROLLARY. The following sequence is exact

$$
\cdots \rightarrow H_{p+1}(W) \stackrel{I}{\rightarrow} H_{p-2 q+1}(V)_{\Delta} \stackrel{\tau}{\rightarrow} H_{p}(W-V) \stackrel{i_{*}}{\rightarrow} H_{p}(W) \rightarrow \cdots .
$$

Proof. The exactness at $H_{p}(W-V)$ follows from Theorem 4.12 and the others are exact by the same proof in Corollary 2.12. Q.E.D. for Corollary 4.17.

In Corollary 4.17, we do not say that if $I\left(\gamma_{p+1}\right)=0$ in $H_{p-2 q+1}(V)$, then $\gamma_{p+1}$ has a representative disjoint from $V$, i.e., we might have $I\left(\gamma_{p+1}\right) \neq 0$ in $H_{p-2 q+1}(V)_{\Delta}$. E.g., let $W=Q_{2}$, the nonsingular quadratic in $\mathrm{CP}_{3}$, i.e., $W=$ $\left\{x^{2}+y^{2}+z^{2}+w^{2}=0\right\} \subset[x, y, z, w]=\mathrm{CP}_{3}$ and $W$ is topologically equivalent to $S^{2} \times S^{2}$ where $S^{2}$ is the two sphere. Let $H_{t}$ be a generic family of hyperplanes and $V_{t}=W \cap H_{t}$, i.e., $H_{t}, t \in \mathbf{C} P_{1}$, is a pencil of hyperplanes in $\mathrm{CP}_{3}$ such that all but two of the $V_{t}$ are nonsingular, in fact quadratic Riemann surfaces, i.e., topologically a two-sphere, and two of them (because degree of $W$ is 2 ), say $V_{0}$ and $V_{1}$, have the equator collapsed to a point, i.e., having a quadratic singularity. Such a family always exists, see. e.g., Moǐsezon [18]. Let $V=V_{0}$ be the hypersurface of $W$. We define a two cycle $\gamma \in H_{2}(W)$ by taking a real line $L$ in the $t$-plane from $t=0$ to $t=1$ and let $\gamma=\bigcup_{t \in L} c_{t}$ where $c_{t}$ is the equator of $V_{t}$ for $t \neq 0,1$ and $c_{0}=c_{1}=$ pinch point (topologically $\gamma \approx S^{2}$ ).

Let $S=\bigcup_{t \in L} S_{t}$ where $S_{t}$ is the upper hemisphere of $V_{t}, t \in L$. If we choose $a, b$ as generators of $H_{2}(W)$ such that $V_{0}=a+b$, then $\partial S=\gamma-(a-b)$. We have $(a-b)$ because if we start at $V_{1}$ with $a$, the upper sphere, then as we move towards $V_{0}$ along $L$ we twist toward the lower sphere $b$ of $V_{0}$ because $W$ is quadratic.

Therefore $\gamma \sim(a-b)$ and $a \cdot a=0=b \cdot b$ and $a \cdot b=1=b \cdot a$. But $(a-b) \cdot(a+b)=0$, i.e., $\gamma \cdot V_{0}=0$, and $a-b$ has no representative disjoint from $V_{0}$, because if it did, then it would have a representative disjoint from $b$, but $(a-b) \cdot b=-1$. Also $(a-b) \cdot(a-b)=-2$, hence $a-b \nsim 0$.

In Corollary 4.17 in this case we have

$$
0 \rightarrow H_{2}(W) \stackrel{I}{\rightarrow} H_{0}\left(V_{0}\right)_{\Delta} \rightarrow H_{1}\left(W-V_{0}\right) \rightarrow 0
$$

where the injection on the left-hand side follows because $a$ and $b$ generate $\mathrm{H}_{2}(W)$ and neither has a representative disjoint from $V_{0}$. We choose as stratum $M_{1,1}=$ $a-$ \{pinch point,$M_{1,2}=b-$ \{pinch point $\}, M_{0}=$ \{pinch point $\}$ and since $a, b$ $\sim 1 / 2 C P_{1}$ in $W$ the $M_{1, i}$ are strata and it is clear they satisfy Theorem 3.2.

Then $a \cdot(a+b)=+1$ implies a representative of $a$ can be chosen so that it intersects $M_{1,2}$ in one point with +1 algebraic intersection. Then for $P \in M_{1,2}$, this implies $\tau_{1}(P) \sim 0$.

Similarly $Q \in M_{1,1}$ implies $\tau_{1}(Q) \sim 0$. Thus $H_{1}\left(W-V_{0}\right)=0$. Then $H_{0}(V)_{\Delta}=Z \oplus Z$ where the generators are $P \in M_{1,2}, Q \in M_{1,1}$ because where 
we can join $P$ to $Q$ by a line $c_{1}$ in $V_{0}$, we cannot construct $\tau\left(c_{1}\right) \subset W-V_{0}$ such that $\partial \tau\left(c_{1}\right)=\tau(P)-\tau(Q)$. We also have $I(\gamma)=(1,-1) \neq 0$ in $H_{0}\left(V_{0}\right)_{\Delta}$, although $I(\gamma)=0$ in $H_{0}\left(V_{0}\right)$. (See Proposition 4.18(a) below.)

We also note that even if $I$ is onto $H_{p-1}(V)$, i.e., $H_{p+1}(W) \stackrel{I}{\rightarrow} H_{p-1}(V)$ $\rightarrow 0$, this does not imply that $\tau$ is the zero map. E.g., let $C$ be the elliptic curve $\left\{x^{3}+y^{3}+z^{3}=0\right\}$ in $\mathbf{C} P_{2}$. Then let $V$ be the cone over $C$ in $\mathbf{C} P_{3}$, i.e., $V=$ $\{x+y+z=0\}$ in $W=\mathbf{C} P_{3}$ with $[x, y, z, w]$ as homogeneous coordinates.

Then $V$ is ample in $\mathrm{CP}_{3}$, hence $H_{1}(V)=0$. Thus $H_{3}\left(\mathrm{CP}_{3}\right) \stackrel{I}{\rightarrow} H_{1}(V) \rightarrow 0$ but if $\alpha_{i}, i=1,2$, are the generators of $H_{1}(C)$, then $\tau\left(\alpha_{i}\right) \propto 0, i=1,2$ in $\mathrm{H}_{2}\left(\mathrm{CP}_{3}-V\right)$ since $\mathrm{H}_{3}\left(\mathrm{CP}_{3}\right)=0$. In fact, $\mathrm{H}_{3}\left(\mathrm{CP}_{3}-V\right)=Z \oplus Z$.

Finally, just as we noted at the end of $\S 2$, these geometric results can be obtained by looking at the Leray spectral sequence induced from the inclusion map $W-V \subset W$.

Again in this spectral sequence we will have $E_{2}^{p, q} \simeq H^{p}\left(W, R^{q}(V)\right)$ where $R^{q}(V)$ is the sheaf obtained from the presheaf $U \rightarrow H^{q}(U-U \cap V)$ for $U$ an open set. Then the stalk of $R^{q}(V)$ at $P$, denoted by $R^{q}(V)_{P}$, is given by proj $\lim H^{q}(U-U \cap V)$ where $U$ is a neighborhood of $P$ in $W$ and we take the inverse limit over all such $U$. We note that a cofinal family of $U$ can be chosen such that $\partial U-\partial U \cap V$ is a strong deformation retract of $U-U \cap V$. This follows from Milnor [17, Lemma 5.9], where he actually shows that $U-U \cap V$ is the cone over $\partial U-\partial U \cap V$.

Then $E_{2}^{p, q} \Rightarrow H^{p+q}(W-V)$.

If $V$ is a hypersurface, let $f$ be a local defining equation of $V$ in $U$. Then Milnor [17] proves that $\partial U-\partial U \cap V=E$ is a locally trivial fibre bundle over a circle with the fibre $F$ being diffeomorphic to $f^{-1}(c) \cap U$ for $c$ a sufficiently small complex number. The fibration is given by $z \mapsto f(z) /|f(z)|$. We have the Wang sequence of the fibration

$$
\rightarrow H_{S}(F) \stackrel{T-I}{\longrightarrow} H_{S}(F) \rightarrow H_{S}(E) \rightarrow H_{S-1}(F) \rightarrow \cdots
$$

where $T$ is the map induced by the action of $\pi_{1}\left(S^{1}\right)=Z$. (Note: $T$ is called the Picard-Lefschetz transform by algebraic geometers) and $I$ denotes the map induced from the identity.

Thus to compute $R^{s}(V)_{P}$, we need only know the action of $T$. This $T$ has been well studied. In case $V$ is a Brieskorn variety at $P$, i.e., $f\left(z_{1}, \ldots, z_{n}\right)=$ $z_{1}^{a_{1}}+\cdots+z_{n}^{a_{n}}$ for $a_{i}>0$, then Pham [21] has computed the action of $T$.

What he does is let $\omega_{i}: \mathbf{C}^{n} \rightarrow \mathbf{C}^{n}$ be multiplying the $i$ th coordinate by a primitive $a_{i}$ th root of unity. Then $\omega_{i}: F \rightarrow F\left(F=f^{-1}(1)\right)$. If we let $e=$ $\left\{\left(z_{1}, \ldots, z_{n}\right) \in F \mid z_{i}\right.$ are real and nonnegative $\}$ (e is homeomorphic to an $(n-1)$-cell) then $\Pi_{i=1}^{n}\left(1-\omega_{i}\right) e=h$ is an $(n-1)$-cycle in $F$ and $F$ is a free group of rank $\prod_{i=1}^{n}\left(a_{i}-1\right)$ with a set of generators given by $\left\{\omega_{1}^{i_{1}} \cdots \omega_{n}^{i_{n}} h \mid\right.$ 
$\left.0 \leqslant i_{j}<a_{j}\right\}$ where the product $\omega_{i} \omega_{j}$ means composition of maps. Furthermore $T$ can be chosen as action $\Pi_{i=1}^{n} \omega_{i}$ where we have the relations $\omega_{i}^{a_{i}}=$ $-\left(1+\omega_{i}+\cdots+\omega_{i}^{a_{i}-1}\right)$.

Another way to compute $T$ is to resolve the singularities of $V$ to normal crossings. $V^{\prime}=U V_{i}$, i.e., when we resolve $V$ we do not change $W-V$ and $E \subset W-V$, so that now $V^{\prime}$ is given by $\Pi_{i=1}^{n} z_{i}^{a_{i}}=0$ where $a_{i} \geqslant 0, a_{i}$ being the multiplicity of $V_{i}$ in $W^{\prime}$ and $z_{i}$ a local defining equation of $V_{i}$. Then $F$ is given by $\Pi_{i=1}^{n} z_{i}^{a_{i}}=1$ and $T: F \rightarrow F$ is given by $\Pi_{i=1}^{n} \omega_{i}$ where $\omega_{i}: \mathbf{C}^{n} \rightarrow \mathrm{C}^{n}$ is multiplying the $i$ th coordinate by a primitive $a_{i}$ th root of unity. Clemens [2] has computed the minimal polynomial of $T$, which is $\Pi_{i=1}^{n}\left(T^{a_{i}}-1\right)$, and given a formula to compute $T^{a}$, where $a=1$.c.m. $\left(a_{1}, \ldots, a_{n}\right)$, if one knows $H_{s}(F)$.

Another way to compute $E$ follows from Alexander duality, viz, $E \subset S^{2 n-1}$ $=\partial U$, so that

$$
\begin{aligned}
H^{s}(E) & \simeq H^{s}\left(S^{2 n-1}-S^{2 n-1} \cap V\right) \\
& \simeq H_{2 n-2-s}\left(S^{2 n-1} \cap V\right)=H_{2 n-2-s}(\tau(P, V))
\end{aligned}
$$

So if one knows $\tau(P, V)$ one is done. If $P$ is an isolated singular point of $V$, then when one resolves singularities to $V^{\prime}=\bigcup V_{i}$, then $\tau(P, V)$ is diffeomorphic to $\tau\left(S, V_{0}\right)$ where $S$ is the singular locus of $V$, and $V_{0}$ is the proper transform of $V$, i.e., $S=\bigcup_{i>0} V_{i} \cap V_{0}$. In many instances this is very easy to compute. The difficulty lies in computing the "multiplicity" of $V_{i} \cap V_{0}$ in $V_{i}$, i.e., the Euler class of the induced normal bundle. In the case when $V$ is a surface, Orlik and Wagreich [20] have computed $\tau(P, V)$ by this method if $V$ admits a $C^{*}$-action. (These include the Brieskorn varieties.) Wagreich [25] has computed $\tau(P, V)$ in case $V$ is what he calls an elliptic singularity.

Let us return to the case of a variety $V$ of codimension $q$ in a complex manifold $W$ of dimension $n$. Then

$$
R^{s}(V)_{P}= \begin{cases}Z, & s=0 \text { for all } P, \text { if } n>2 \\ 0, & s>0, P \in W-V, \\ Z, & s=2 q-1, P \in V-S \\ 0, & 0<s<2 q-1, P \in V \\ 0, & s \geqslant n+1, P \in S \text { and } V \text { is a hypersurface. }\end{cases}
$$

For $n=2, R^{0}(V)_{P}$ will equal the free group on the number of components of $V$ at $P$. By Alexander duality, $H^{s}(E) \simeq H_{2 n-2-s}(\tau(P, V))$ and $\tau(P, V)$ is of dimension $2 n-2 q-1$. If $V$ is a hypersurface, Milnor [17] has shown that $\tau(P, V)$ is $(n-3)$-connected. He has also shown that $F$ has the homotopy type of a finite $\mathrm{CW}$-complex of real dimension $n-1$.

Suppose $V$ has only isolated singularities $\left\{P_{i}\right\}$ and $n>2$. Then $R^{s}(V)_{P_{i}} \simeq$ 
$H^{s-2 q+1}\left(\tau\left(P_{i}, V\right)\right)$ by Alexander and Poincaré duality. Then $R^{2 q-1}(V) \simeq Z_{V}$, i.e., the constant sheaf whose stalk over each point of $V$ is $Z$ and whose stalk off of $V$ is zero, so that $E_{2}^{p, 0} \simeq H^{p}(W), E_{2}^{p, 2 q-1} \simeq H^{p}(V)$ and $E_{2}^{p, s}=0$ for $p>1$ and $s \neq 0,2 q-1$.

$$
E_{2}^{0, s}=\bigoplus_{i} H^{0}\left(P_{i}, R^{s}(V)_{P_{i}}\right) \simeq \bigoplus_{i} H^{s-2 q+1}\left(\tau\left(P_{i}, V\right)\right)
$$

where the last isomorphism follows from Alexander-Poincaré duality.

4.18 Proposition. If $V$ only has isolated singularities and is of codimension $q$, then

(a) $E_{2}^{p, 0} \Rightarrow$ image $\left\{H_{p}(W-V) \rightarrow H_{p}(W)\right\}$;

(b) $E_{2}^{p-2 q+1,2 q-1} \Rightarrow$ image $\left\{H_{p-2 q+1}(V)_{\Delta} \rightarrow H_{p-2 q+1}(V)\right\}$, i.e., those $\gamma_{p-2 q+1}$ of $H_{p-2 q+1}(V)_{\Delta}$ which do not bound in $V$;

(c) $E_{2}^{0, p} \Rightarrow \operatorname{ker}\left\{H_{p-2 q+1}(V)_{\Delta} \rightarrow H_{p-2 q+1}(V)\right\}$.

Proof. (a) ker $d_{2}^{p, 0}=E_{2}^{p, 0}=H^{p}(W)$. The first nonzero term in the spectral sequence to map onto $E_{r}^{p, 0}$ is

$$
E_{2 q}^{p-2 q, 2 q-1}=H^{p-2 q}(V) \rightarrow H^{p}(W)=E_{2 q}^{p, 0}
$$

and $d_{2 q}^{p-2 q, 2 q-1}$ is adjoint to intersection $H_{p}(W) \stackrel{I}{\rightarrow} H_{p-2 q}(V)$ so that coker $d_{2}^{p-2 q, 2 q-1}=\operatorname{ker} I$, i.e., $E_{2 q+1}^{p, 0}=\left\{\gamma_{p} \in H_{p}(W) \mid \gamma_{p} \cdot V=0\right\}$.

We know from the above examples that a cycle having a zero intersection with $V$ does not guarantee that it has a representative disjoint from $V$, but this is a first approximation.

The next nonzero term to map onto $E_{r}^{p, 0}$ is

$$
d_{p}^{0, p-1}: E_{p}^{0, p-1}=\bigoplus_{i} H^{p-2 q}\left(\tau\left(P_{i}, V\right)\right) \rightarrow E_{2 q+1}^{p, 0} \subset H^{p}(W)
$$

and this is also adjoint to the map $H_{p}(W) \rightarrow\left\{\bigoplus_{i} H_{p-2 q}\left(\tau\left(P_{i}, V\right)\right)\right\} \subset$ $H_{p-2 q}(V)$ given by intersection in $V$. The kernel of this intersecting map are those $p$-cycles of $W$ which have zero intersection with $\tau\left(P_{i}, V\right)$, which means precisely that if $\gamma_{p} \cap V=\gamma_{p-2 q}=\partial c_{p-2 q+1}$ for $c_{p-2 q+1} \subset V$, then $c_{p-2 q+1}$ can be chosen to miss the $P_{i}$; for if not, then $\gamma_{p-2 q}$ is a cone over $P_{i}$, i.e., $0 \neq \gamma_{p-2 q} \in H_{p-2 q}\left(\tau\left(P_{i}, V\right)\right)$.

Thus we get image $d_{p}^{0, p-1}=\left\{\gamma_{p} \in H_{p}(W) \mid \gamma_{p} \cdot V=\gamma_{p-2 q}=\partial c_{p-2 q+1}\right.$ but $c_{p-2 q+1}$ cannot be pulled off some $P_{i}$ \}, i.e.,

$$
E_{\infty}^{p, 0}=\text { image }\left\{H_{p}(W-V) \rightarrow H_{p}(W)\right\} .
$$

(b) $E_{2}^{p-2 q+1,2 q-1}=H^{p-2 q+1}(V) \rightarrow E_{2}^{p-2 q+2,2 q-2}$ will be the zero map until

$$
E_{2 q}^{p-2 q+1,2 q-1}=H^{p-2 q+1}(V) \rightarrow H^{p+1}(W)=E_{2 q-1}^{p+1,0}
$$


which is adjoint to intersection, so

$$
\begin{aligned}
\operatorname{ker} d_{2 q}^{p-2 q+1,2 q-1}=\left\{\gamma_{p-2 q+1}\right. & \in H_{p-2 q+1}(V) \mid \gamma_{p-2 q+1} \\
\neq \gamma_{p+1} & \left.\cap V \text { for } \gamma_{p+1} \in H_{p+1}(W)\right\}
\end{aligned}
$$

There will not be terms which map onto $E_{r}^{p-2 q+1,2 q-1}$ until

$$
E_{p-2 q+1}^{0, p-1}=\bigoplus_{i} H^{p-2 q}\left(\tau\left(P_{i}, V\right)\right) \rightarrow E_{p-2 q+1}^{p-2 q+1,2 q-1} \subset H^{p-2 q+1}(V)
$$

which is adjoint to the intersection map. Thus

$$
\begin{aligned}
E_{p-2 q+2}^{p-2 q+1,2 q-1} & =E_{\infty}^{p-2 q+1,2 q-1} \\
& =\left\{\gamma_{p-2 q+1} \in H_{p-2 q+1}(V) \mid \gamma_{p-2 q+1} \in H_{p-2 q+1}(V)_{\Delta}\right\}
\end{aligned}
$$

since we can construct the tube over $\gamma_{p-2 q+1}$ if and only if $\gamma_{p-2 q+1}$ misses all the $P_{i}$ if and only if $\gamma_{p-2 q+1} \cap \tau .\left(P_{i}, V\right)$ is zero in $\tau\left(P_{i}, V\right)$.

(c) $d_{2}^{0, p}: E_{2}^{0, p} \rightarrow E_{2}^{2, p-1}$ will be the zero map until we reach $E_{r}^{p-2 q+2,2 q-1}$, i.e.,

$$
\begin{aligned}
E_{p-2 q+2}^{0, p} & =E_{2}^{0, p} \\
& =\bigoplus_{i} H^{p-2 q+1}\left(\tau\left(P_{i}, V\right)\right) \stackrel{d_{p-2 q+2}^{0, p}}{\longrightarrow} E_{p-2 q+2}^{p-2 q+2,2 q-1} \\
& \simeq H^{p-2 q+2}(V)
\end{aligned}
$$

which is adjoint to the intersection map $I_{0, p}$. Also

$$
\bigoplus_{i} H^{p-2 q+1}\left(\tau\left(P_{i}, V\right)\right) \supset E_{p-2 q+3}^{0, p}=E_{p+1}^{0, p} \stackrel{d_{p+1}^{0, p}}{\longrightarrow} H^{p+1}(W)
$$

is adjoint to the intersection map with $V$, so that $d_{p-2 q+2}^{0, p}$ and $d_{p+1}^{0, p}$ together give those cycles $\Sigma_{i} \gamma_{p-2 q+1, i} \in \bigoplus_{i} H_{p-2 q+1}\left(\tau\left(P_{i}, V\right)\right)$ such that $\Sigma_{i} \tau\left(\gamma_{p-2 q+1, i}\right) \neq 0$ in $W-V$, i.e., $\Sigma_{i} \gamma_{p-2 q+1, i} \in$ image $I_{0, p}$ implies $\Sigma_{i} \gamma_{p-2 q+1, i}=\Sigma_{i} \tau\left(P_{i}, V\right) \cap \gamma_{p-2 q+2}$ for $\gamma_{p-2 q+2} \in H_{p-2 q+2}(V)$ so that

$$
\sum_{i} \tau\left(\gamma_{p-2 q+1, i}\right)=-\partial \tau\left(\gamma_{p-2 q+2}-\sum \tau\left(P_{i}, V\right) \cap \gamma_{p-2 q+2}\right) \sim 0
$$

in $W-V$ while $d_{p}^{0, p+1}\left(\Sigma_{i} \gamma_{p-2 q+1, i}\right)=0$ if and only if $\Sigma_{i} \tau\left(\gamma_{p-2 q+1, i}\right)=0$. Therefore

$$
E_{p+2}^{0, p} \simeq E_{\infty}^{0, p}=\operatorname{ker}\left\{H_{p-2 q+1}(V)_{\Delta} \rightarrow H_{p-2 q+1}(V)\right\}
$$

because $H_{p-2 q+1}\left(\tau\left(P_{i}, V\right)\right) \rightarrow H_{p-2 q+1}(V)$ has zero image. Q.E.D.

In general, if $V$ does not have isolated singularities, we have that $H_{2 n-2 q-1}\left(\tau\left(P_{i}, V\right)\right) \simeq R^{2 q-1}(V)_{P_{i}} \simeq$ free group on the number of components 
$V$ has at $P_{i}$. Thus $E_{2}^{p, 2 q-1} \simeq \bar{H}^{p}(V)$ where the "-" over $H$ means we count the multiplicity of the number of components of $V$ at each point, i.e. $\bar{H}^{p}(V) \simeq$ $\Sigma_{i} H^{p}\left(V_{i}\right)$ where $V_{i}$ are the (local) irreducible components of $V$. Also $R^{s}(V)=0$ for $0<s<2 q-1$. Also support $R^{s}(V) \subseteq S$, singular locus of $V$ for $s \geqslant 2 q-1$, so that $E_{2}^{p-s, s} \simeq H^{*}\left(\tau\left(S^{\prime}, V\right)\right)$ for some subvariety $S^{\prime}$ of $S$.

\subsection{COROLlary. Let $V$ be an arbitrary subvariety of codimension $q$,} then:

(a) $E_{2}^{p, 0} \Rightarrow$ image $\left\{H_{p}(W-V) \rightarrow H_{p}(W)\right\}$;

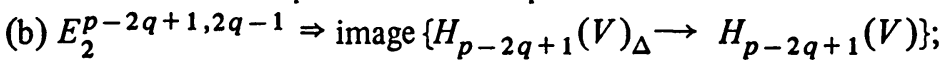

(c) for $s>2 q-1$,

$$
E_{2}^{p-s, s} \Rightarrow \operatorname{ker}\left\{H_{p-2 q+1}(V)_{\Delta} \rightarrow H_{p-2 q+1}(V)\right\} .
$$

Thus Theorem 4.12 is a study of (b) and (c) while Corollary 4.17 studies (c).

Furthermore, Corollary 4.17 gives us an intersection pairing on $V$, a variety of $\operatorname{dim} q$, i.e., there is a natural mapping $H^{p}(V) \rightarrow H_{2 q-p}(V)_{\Delta}$ which is an isomorphism, and also a group $H^{p}(V)_{\Delta}$ can be defined which corresponds to a cohomology theory to the homology theory such that $H_{p}(V) \rightarrow H^{2 q-p}(V)_{\Delta}$ is also an isomorphism. This gives us an intersection pairing on $V$ just as Poincaré duality does for nonsingular $V$. Also $H_{p}(V)_{\Delta}$ can be shown to be an intrinsic property of $V$, i.e., independent of the embedding of $V$ into $W$, ambient manifold. In fact, if $V$ is any polyhedra embedded as a subcomplex of some $C^{\infty}$-manifold $W$, then $H_{q}(V)_{\Delta}$ (resp. $H^{q}(V)_{\Delta}$ ) can be constructed such that Corollary 4.17 is true and we have the duality theorem. For details, see Gordon [6].

5. The residue operator. If $V$ is a hypersurface of a complex manifold $W$, then we have shown that we have an exact sequence in Corollaries 2.12 or 4.17 which reduces to the Thom-Gysin sequence in case $V$ is nonsingular. We can dualize the sequence, i.e., take Hom, and since our coefficients are over a field $\mathbf{C}$, we get the long exact sequence in cohomology,

$$
\cdots \rightarrow H^{p}(W) \stackrel{i^{*}}{\rightarrow} H^{p}(W-V) \stackrel{R}{\rightarrow} H^{p-1}(V)_{\Delta} \stackrel{\bigcup[V]^{*}}{\rightarrow} H^{p+1}(W) \rightarrow \cdots
$$

where $H^{p-1}(V)_{\Delta}$ is by definition equal to $\operatorname{Hom}_{\mathrm{C}}\left(H_{p-1}(V)_{\Delta}, \mathbf{C}\right)=H_{p-1}(V)_{\Delta}^{*}$. The map $U[V]^{*}$ is essentially cup product with the cohomology class of $V$, i.e., $V$ defines a homology class $[V] \in H_{2 n-2}(W)$ and hence by Poincare duality, has a dual two-dimensional cohomology class $[V]^{*}$.

The map $R$ is the Poincare residue operator in case $V$ is nonsingular. We know that if $\omega \in H^{p}(W-V)$, then by de Rham's theorem we can represent $\omega$ by a closed differential form which is at least $C^{\infty}$ in $W-V$. Furthermore the representation is unique up to exact such forms. 
Leray [14] has shown that if $V$ is nonsingular, then $\omega$ has a representation of the form $\theta \wedge \lambda+\eta$ where $\theta$ and $\eta$ are smooth forms of $W$, i.e., they are $C^{\infty}$ with no singularities. If $j: V \rightarrow W$ is the inclusion map, then $j^{*} \theta=\theta \mid V$ is a closed form of $V$ and $\lambda$ has a pole of order one on $V$, i.e.,

5.1 Definition. A differential form $\lambda$ has a pole of order $k$ on $V$ if $\left(f_{U}\right)^{k} \cdot \lambda$ is smooth in $U$, where $U$ is any coordinate chart of $W$ and $f_{U}$ is a local defining equation of $V$ in $W$.

In fact Leray shows that if $\{U\}$ is a locally finite cover of $W$ by coordinate charts and $\left\{e_{U}\right\}$ is a partition of unity subordinate to $\{U\}$, then $\lambda$ can be chosen to have the form $\Sigma_{U} e_{U} d f_{U} / f_{U}$. Since the $f_{U}$ are analytic, this implies $\lambda$ is a $(1,0)$ form and $d \lambda$ is essentially the Poincare dual of $[V]$, i.e., if $\Omega$ is a fundamental $(n-1, n-1)$-form of $V$, then $\int_{W} \Omega \wedge d \lambda>0$. Then Leray proves $R(\omega)$ $=j * \theta / 2 \pi \sqrt{-1}$.

Hence to analyze $R$ in (3), we will first consider the case of normal crossings.

5.2 THEOREM (RoBIN). If $V$ has normal crossings, then $H^{*}(W-V)$ can be represented by differential forms having a pole of order one on $V$. In fact, if $\{U\}$ is a locally finite cover of $W$ by coordinate charts, $\left\{e_{U}\right\}$ a partition of unity subordinate to the cover $\{U\}$ and $f_{U}$ a local defining equation of $V$ in $U$, then $\alpha \in H^{*}(W-V)$ has a representation $\omega$, a closed form, of the type $\omega=$ $\left(\theta \wedge \Sigma_{U} e_{U} d f_{U} / f_{U}\right)+\eta$ where $\theta \wedge e_{U} d f_{U}$ and $f_{U} \cdot \eta$ are smooth.

Proof OF THEOREM 5.2. Robin [23] has shown that each class is represented by a form having a pole of order one, and Leray [14] has shown that if a closed form $\omega$ of degree $p$ in $W-V$ has a pole of order one on $V$, then $\omega$ has a representation in $U$ of the type

$$
\theta_{p}+\sum_{q=1}^{p-1} \sum_{\left\{i_{1}, \ldots, i_{q}\right\}} \theta_{i_{1}} \cdots i_{q} \wedge \frac{d f_{i_{1}, U}}{f_{i_{1}, U}} \wedge \cdots \wedge \frac{d f_{i_{q}, U}}{f_{i_{q}, U}}
$$

where the $\theta$ 's are smooth and $f_{U}=\prod_{i=1}^{k} f_{i, U}$. But

$$
\sum_{i=1}^{k} \frac{d f_{i, U}}{f_{i, U}}=\frac{d f_{U}}{f_{U}}
$$

hence the representation as stated in the theorem. Q.E.D for Theorem 5.2.

Note. The choice of $\theta$ is not unique, e.g., if $f(x, y)=x y$ defines $V$ in $W=$ $\mathrm{C}^{2}=(x, y)$, then $H^{2}(W-V)$ can be represented by $d x / x \wedge d f / f$ as well as by $\theta_{1} \wedge d f / f$ where $\theta_{1}=(\bar{x} d \bar{x}-\bar{y} d \bar{y} /(x \bar{x}+y \bar{y})$.

Note. Suppose we let

$$
\Psi_{q}=\sum_{i_{1} \cdots i_{q}} \theta_{i_{1}} \cdots i_{q} \wedge \frac{d f_{i_{1}, U}}{f_{i_{1}, U}} \wedge \cdots \wedge \frac{d f_{i_{q}, U}}{f_{i_{q}, U}},
$$


using the notation of the proof of Theorem 5.2. Let $\gamma_{p-q} \in H_{p-q}\left(\bar{M}_{q}\right)_{\Delta}$, i.e., we have $\tilde{\tau}_{1} \cdots \tilde{\tau}_{q} \gamma_{p-q} \in H_{p}(W-V)$. We would like to say that the homology class which has period one on $\tilde{\tau}_{1} \cdots \tilde{\tau}_{q} \gamma_{p-q}$ and zero periods on the other classes can be represented by a form which is locally of the type $\Psi_{q}+\eta$ where $\eta$ is smooth, i.e., we do not need $\Psi_{r}$ for $r \neq q$.

But consider, for example, $V=V_{1} \cup V_{2}$ and $\gamma_{p-2} \in H_{p-2}\left(V_{1} \cap V_{2}\right)$. Suppose $\theta_{12}$ is a closed form on $V_{12}$ which represents the dual cohomology class of $\gamma_{p-2}$ via integration. Then let $\theta_{12}^{\prime}$ be a smooth form on $W$ which vanishes off $T_{1,1} T_{2,2} V_{12}$ and equals $\theta_{12}$ near $V_{12}$; and let $f_{i}$ be a local defining equation of $V_{i}$. Then we would like to say that $\theta_{12}^{\prime} \wedge d f_{1} / f_{1} \wedge d f_{2} / f_{2}$ should (modulo a smooth form on $W$ ) represent the dual cohomology class of $\tau_{1} \tau_{2} \gamma_{p-2}$.

One can show that $d\left(\theta_{12}^{\prime} \wedge d f_{1} / f_{1} \wedge d f_{2} / f_{2}\right)$ has no periods on $W$, but it is not necessarily smooth, i.e., $d \theta_{12}^{\prime}$ is closed near $V_{12}$, but $d \theta_{12}^{\prime} \mid V_{1}$ is not zero, hence $d \theta_{12}^{\prime} \wedge d f_{1} / f_{1} \wedge d f_{2} / f_{2}$ need not be smooth on $V_{1}$. Hence, we might need a correction term, $\theta_{1} \wedge d f_{1} / f_{1}$, to make it smooth on $V_{1}$, and another correction term, $\theta_{2} \wedge d f_{2} / f_{2}$, to make it smooth on $V_{2}$, where the $\theta_{i}$ are smooth forms.

However, we would have that $R\left(\theta_{i} \wedge d f_{i} / f_{i}\right)=0$. This is because in the spectral sequence of the map $j: W-V \subset W$, we showed that $E_{2}^{p-1,1} \Rightarrow$ $\tau_{1} H_{p-1}\left(\bar{M}_{1}\right)_{\Delta}$, while $E_{2}^{p-2,2} \Rightarrow \tau_{1} \tau_{2} H_{p-2}\left(\bar{M}_{2}\right)_{\Delta}$. But we are working over a field, hence there are no relations among $E_{\infty}^{p-1,1}$ and $E_{\infty}^{p-2,2}$. But if $R\left(\theta_{i} \wedge d f_{i} / f_{i}\right) \neq 0$ and $\theta_{i} \wedge d f_{i} / f_{i}$ is part of the dual cohomology class of $\tau_{1} \tau_{2} \gamma_{p-2}$, these would give nontrivial relations.

Hence, to analyze $R$ in (3) when $V$ has normal crossings, we have that if $\gamma_{p-q} \in H_{p-q}\left(\bar{M}_{q}\right)_{\Delta}$ and $\alpha$ is the dual homology class of $\tilde{\tau}_{1} \cdots \tilde{\tau}_{q} \gamma_{p-q}$, then

$$
R(\alpha)=(2 \pi \sqrt{-1})^{q} \sum_{i_{1} \cdots i_{q}}\left[\theta_{i_{1}} \cdots i_{q} \mid V_{i_{1}} \cap \cdots \cap V_{i_{q}}\right] \in H^{p-q}\left(\bar{M}_{q}\right)_{\Delta} .
$$

Thus $R$ is a mapping on differential forms of degree $p$ to differential forms of degree less than or equal to $p-1$. If we represent $\omega$ by

$$
\omega=\theta^{\prime} \wedge \sum_{U} e_{U} \frac{d f_{U}}{f_{U}}+\eta
$$

then $R(\omega)=(2 \pi \sqrt{-1}) \theta^{\prime} \mid V$ where $\theta^{\prime}$ is a closed $(p-1)$-form on $V$ with singularities (in fact logarithm singularities) on the singular locus of $V$, i.e., $R$ maps closed $p$-forms with poles on $V$ to closed $(p-1)$-forms with (perhaps) poles on the singular locus of $V$.

Furthermore $R$ commutes with $d$, i.e. $R(d \omega)=d R(\omega)$, because if $\lambda=$ $\Sigma_{U} e_{U} d f_{U} / f_{U}$ then $d \lambda$ is smooth, in fact $d \lambda$ is a $(1,1)$ form which is the Poincare dual of $V$ in $W$. We also remark that this description of $R$ is independent of 
the representation of $\omega$, i.e., if $\omega=\theta^{\prime \prime} \wedge \Sigma_{U} e_{U} d f_{U} / f_{U}+\eta$, then $\theta^{\prime}\left|V=\theta^{\prime \prime}\right| V$, and if $f_{U}^{\prime}$ is another defining equation, i.e., $f_{U}^{\prime}=g_{U} f_{U}$ where $g_{U}$ is nonvanishing in $U$, then $\omega=\theta^{\prime} \wedge \Sigma e_{U} d f_{U}^{\prime} / f_{U}^{\prime}+\eta$ will have $R(\omega)=\theta^{\prime} \mid V$ as well since $d f_{U}^{\prime} / f_{U}=d f_{U} / f_{U}+d g_{U} / g_{U}$ and $g_{U}$ is nonvanishing.

Let us state this explicitly.

5.3 CoROllaRY. Let $V$ have normal crossings and $\lambda=\Sigma_{U} e_{U} d f_{U} / f_{U}$ and $\omega \in H^{p}(W-V)$. Then in (3), $R(\omega)=\theta \mid V$, a closed form on $V-S$ with (perhaps) a pole of order one on $S$, and $U[V]^{*}$ is essentially the wedge product with $d \lambda$, a smooth form.

Note. We say essentially because we actually have

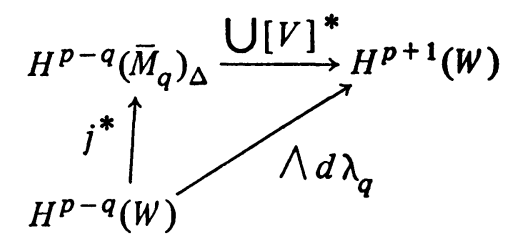

where

$$
\lambda_{q}=\sum_{U} e_{U} \frac{d f_{1, U} \wedge \cdots \wedge d f_{q, U}}{f_{.1, U} \cdots f_{q, U}}
$$

where $\bar{M}(1, \ldots, q)$ is given by $\bigcap_{i=1}^{q}\left(f_{i, U}=0\right)$ in $U . d \lambda_{q}$ will be a $(q, 1)$-form and $j^{*}$ is the restriction map, i.e., $j: \bar{M}_{q} \subset W$.

We would like to say that if $V$ is any hypersurface and $\lambda=\Sigma_{U} e_{U} d f_{U} / f_{U}$ where the $\{U\}$ are suitably chosen coordinate charts, then $\alpha \in H^{*}(W-V)$ has a representative of the form $\omega=\theta \wedge \lambda+\eta$ where $f_{U} \cdot \theta \wedge \lambda$ and $f_{U} \cdot \eta$ are smooth with $R(\alpha)=[\theta \mid V]$. Unfortunately, proving it for the normal crossings case does not suffice.

Suppose, then, we have given $V \subset W$ and we take a resolution $\left(W^{\prime}, V^{\prime}, \pi\right)$ where $V^{\prime}=\pi^{-1}(V), \pi: W^{\prime} \rightarrow W$ and $V^{\prime}$ has local normal crossings. Then if $U$ is a local coordinate chart in $W$ with $f_{U}$ a local defining equation of $V$ and $\pi^{-1}(U)=U^{\prime}$, then we set $f_{U} \circ \pi=f_{U^{\prime}}$ and $f_{U^{\prime}}=\Pi_{i=1}^{k}\left(f_{i, U^{\prime}}\right)^{\alpha_{i}}$ with $\alpha_{i} \geqslant 1$ and $\Pi_{i} f_{i, U^{\prime}}$ is a local defining equation of $V^{\prime}$ in $U^{\prime}$.

Let $\lambda=\Sigma_{U} e_{U} d_{U} / f_{U}$ for $\{U\}$ a suitably chosen locally finite cover of $W$. Then $\pi^{-1}(U)=U^{\prime}$ is a locally finite cover of $W^{\prime}$ and one can choose a refinement $\left\{U^{\prime \prime}\right\}$ of $\left\{U^{\prime}\right\}$ with $\left.f_{U^{\prime \prime}}\right|_{U^{\prime} \cap U^{\prime \prime}}=\left.f_{U^{\prime}}\right|_{U^{\prime} \cap U^{\prime \prime}}$ such that $U^{\prime \prime}$ are coordinate charts which satisfy the representation of $\omega$ in Theorem 5.2. Then $d f_{U^{\prime \prime}} / f_{U^{\prime \prime}}=$ $\sum_{i=1}^{k} \alpha_{i} d f_{i, U^{\prime \prime}} / f_{i, U^{\prime \prime}}$. But in the proof of Theorem 5.2, one can replace all of the $d f_{i, U^{\prime \prime}} / f_{i, U^{\prime \prime}}$ by $\alpha_{i} d f_{i, U^{\prime \prime}} / f_{i, U^{\prime \prime}}$ and the conclusions will still be valid, i.e., since the $\alpha_{i}$ are invariant of the coordinate chart representation, one has only multiplied the representative by a nonzero constant. Hence one will have by The- 
orem 5.2 that $\alpha \in H^{*}\left(W^{\prime}-V^{\prime}\right)$ has a representative $\omega^{\prime}=\theta^{\prime} \wedge \lambda^{\prime}+\eta^{\prime}$ where $\lambda^{\prime}=\Sigma_{U^{\prime \prime}} e_{U^{\prime \prime}} d f_{U^{\prime \prime}} / f_{U^{\prime \prime}}$. But if $I_{U}=\left\{U^{\prime \prime} \mid U^{\prime \prime} \subset \pi^{-1}(U)\right\}$, then $U_{I_{U}} \pi^{-1}\left(U^{\prime \prime}\right)$ $=U^{\prime}$ and $\Sigma_{I_{U}} e_{U^{\prime \prime}} f_{U^{\prime \prime}}=e_{U^{\prime}} f_{U^{\prime}}=\left(e_{U} \circ \pi\right)\left(f_{U}^{\circ} \pi\right)$ so that $\pi^{*} \lambda=\lambda^{\prime}$.

Hence $\left(\pi^{*}\right)^{-1} \omega=\left(\pi^{*}\right)^{-1} \theta^{\prime} \wedge \lambda^{\prime}+\left(\pi^{*}\right)^{-1} \eta^{\prime}$. If $S$ denotes the singular locus of $V$, and $S^{\prime}=\pi^{-1}(S)$, then $\pi \mid W^{\prime}-S^{\prime}$ is a bianalytic isomorphism so that $\left(\pi^{*}\right)^{-1}\left(\theta^{\prime}\right)$ is a form on $V-S$ which is smooth as $\theta^{\prime} \mid W^{\prime}-S^{\prime}$ is smooth and $\theta^{\prime}$ is closed near $V^{\prime}$ implies $\left(\pi^{*}\right)^{-1} \theta^{\prime}$ is closed near $V-S$.

Hence, we have shown

5.4 Proposition. If $V$ is a hypersurface in $W$ and $\alpha \in H^{*}(W-V)$, then $\alpha$ has a representative of the form $\theta \wedge \lambda+\eta$ where $\lambda=\Sigma_{U} e_{U} d f_{U} / f_{U}$. Moreover, $R(\alpha)=[\theta \mid V]$, which will be a closed form with (perhaps) singularities on $S$, the singular locus of $V$.

However, we cannot say that $f_{U} \cdot\left(\pi^{*}\right)^{-1} \eta^{\prime}$ is smooth, i.e., if $P \in S$, then we can have $P_{i}, P_{i}^{\prime} \in W-S$ with $P_{i}, P_{i}^{\prime} \rightarrow P$ but $\lim \eta^{\prime}\left(P_{i}\right) \neq \lim \eta^{\prime}\left(P_{i}^{\prime}\right)$. Also, we cannot say that $f_{U} \cdot\left(\pi^{*}\right)^{-1} \theta^{\prime} \wedge \lambda$ is smooth as $\left(\pi^{*}\right)^{-1} \theta^{\prime}$ may acquire singularities, even if $\theta$ is smooth, as in the example after Corollary 4.15 we have $\gamma_{1} \nsim 0$ in $V^{\prime}$ hence $\theta^{\prime} \mid V^{\prime}$ is smooth, but $\gamma_{1} \sim 0$ in $V$ so that $\left(\pi^{*}\right)^{-1} \theta^{\prime} \mid V$ will have a singularity at the singular point of $V$. But in this example, $f_{U}\left(\pi^{*}\right)^{-1} \theta^{\prime} \wedge$ $\lambda$ is smooth as $\lambda$ has a zero at the singular point of sufficiently high order to make the form smooth.

However, we can say if $\Phi^{p}(U)$ denotes the closed, smooth $p$-forms in $U$, $\Omega(U)$ are smooth forms in $U$, and $d \Omega(U)$ are exact ones, then

5.5 Proposition. Let $\theta \in \Phi^{p}(W-V)$ and $\left(W^{\prime}, V^{\prime}, \pi\right)$ a resolution of $(W, V)$ whose centers are nonsingular. Then if $\pi^{*} \theta$ is smooth, either

(i) $\theta-d \eta \in \Phi^{p}(W)$ for some $\eta \in \Omega^{p-1}(W-V)$ or

(ii) $\theta \in d \Omega(W-V)$.

Proof. By Proposition 3.1, since $\operatorname{Ker} i_{*}=R_{p}(V) \simeq R_{p}\left(V^{\prime}\right)=\operatorname{Ker} i_{*}^{\prime}$ where $i: V \subset W, i^{\prime}: V^{\prime} \subset W^{\prime}$, we have that (as coefficients are in C) $\pi^{*}$ : Coker $i^{*}$ $\simeq \operatorname{Coker}\left(i^{\prime}\right)^{*}$, i.e., $\pi^{*}: \operatorname{Ker} R \simeq \operatorname{Ker} R^{\prime}$ where

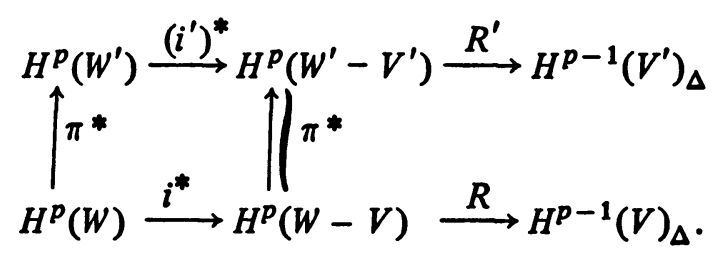

Hence $\theta$ closed implies $[\theta] \in H^{p}(W-V)$ and $\pi^{*}[\theta]$ being smooth implies $R^{\prime} \pi^{*}[\theta]=0$ which implies $R[\theta]=0$, i.e., either $\theta-d \eta \in \Phi(W)$ or $\theta \in$ $d \Omega(W-V)$. Q.E.D. for Proposition 5.5. 
5.6 LEMMA. $d\left(f_{U} \cdot\left(\pi^{*}\right)^{-1}\left(\eta^{\prime}\right)\right)$ has no periods on $W$.

Proof of Lemma 5.6. Let $\left[\gamma_{p+1}\right] \in H_{p+1}(W)$, then $\gamma_{p+1} \cap V=\gamma_{p-1}$ with $\left[\gamma_{p-1}\right] \in H_{p-1}(V)_{\Delta}$. Hence,

$$
\begin{aligned}
\int_{\gamma_{p+1}} d\left(f_{U} \cdot\left(\pi^{*}\right)^{-1}\left(\eta^{\prime}\right)\right) & =\lim _{\epsilon \rightarrow 0} \int_{\gamma_{p+1}-T_{\epsilon} \gamma_{p-1}} d\left(f_{U} \cdot\left(\pi^{*}\right)^{-1}\left(\eta^{\prime}\right)\right) \\
& =-\lim _{\epsilon \rightarrow 0} \int_{\tau_{\epsilon} \gamma_{p-1}} f_{U} \cdot\left(\pi^{*}\right)^{-1}\left(\eta^{\prime}\right)
\end{aligned}
$$

by Stokes' theorem where $T_{\epsilon} \gamma_{p-1}$ are all the fibres of length less than or equal to $\epsilon$ and $\tau_{\epsilon} \gamma_{p-1}$ are all the fibres of length equal to $\epsilon$.

By Proposition 3.1, $\pi^{*} \tau_{\epsilon} \gamma_{p-1}=\tau_{\epsilon} \gamma_{p-1}^{\prime}$ for some $\gamma_{p-1}^{\prime} \in H_{p-1}\left(V^{\prime}\right)_{\Delta}$, i.e., in the proof of that proposition, it was shown that $\pi^{-1}\left(\gamma_{p-1}\right) \in$ $Z_{p-1}\left(V^{\prime}\right)_{\Delta}$ if $\gamma_{p-1} \in H_{p-1}(V)_{\Delta}$. Hence

$$
-\lim _{\epsilon \rightarrow 0} \int_{\tau_{\epsilon} \gamma_{p-1}} f_{U} \cdot\left(\pi^{*}\right)^{-1}\left(\eta^{\prime}\right)=-\lim _{\epsilon \rightarrow 0} \int_{\tau_{\epsilon} \gamma_{p-1}^{\prime}} \pi^{*}\left(f_{U}\right) \cdot \eta^{\prime}=0
$$

since $\pi^{*} f_{I r}=f \cdot f_{U}^{\prime}$ and $f_{U}^{\prime} \cdot \eta^{\prime}$ is smooth, while $\lim _{\epsilon \rightarrow 0} \tau_{\epsilon} \gamma_{p-1}^{\prime}$ is a $(p-1)$ dimensional chain. Q.E.D. for Lemma 5.6.

5.7 Proposition. If $d\left(f_{U} \cdot\left(\pi^{*}\right)^{-1}\left(\eta^{\prime}\right)\right)$ is smooth, then $\alpha \in H^{*}(W-V)$ has a representative of the form $\theta \wedge \lambda+\eta$ where $f_{U} \cdot \eta$ is smooth.

Proof of Proposition 5.7. If $d\left(f_{U} \cdot\left(\pi^{*}\right)^{-1}\left(\eta^{\prime}\right)\right)$ is smooth, then by Lemma 5.6 and de Rham's theorem, we have a $\xi \in \Omega(W)$ such that $d\left(f_{U} \cdot\left(\pi^{*}\right)^{-1}\left(\eta^{\prime}\right)\right)=d \xi$. Hence $f_{U} \cdot\left(\pi^{*}\right)^{-1}\left(\eta^{\prime}\right)-\xi$ is a closed form on $W-$ $V$ such that $\pi^{*}\left(f_{U} \cdot\left(\pi^{*}\right)^{-1}\left(\eta^{\prime}\right)-\xi\right)$ is smooth, so that by Proposition 5.5 either

$$
f_{U} \cdot\left(\pi^{*}\right)^{-1}\left(\eta^{\prime}\right)-\xi-d \rho=\Psi \in \Omega(W)
$$

or

$$
f_{U} \cdot\left(\pi^{*}\right)^{-1}\left(\eta^{\prime}\right)-\xi=d \rho \quad \text { for } \rho \in \Omega(W-V) .
$$

Then let $\omega=\left(\pi^{*}\right)^{-1} \omega^{\prime}-d \rho=\left(\pi^{*}\right)^{-1} \theta^{\prime} \wedge \lambda+\eta$ where $\eta$ is now smooth. If we set $\theta=\left(\pi^{*}\right)^{-1} \theta^{\prime}$, then $\theta$ is a smooth form on $W-S$ with $\theta \mid V$ being closed. Q.E.D. for Proposition 5.7.

5.8 Conjecture. If $V$ is a hypersurface in $W$, a complex manifold and $\alpha \in H^{*}(W-V)$, then $\alpha$ has a representative of the form $\omega=\theta \wedge \lambda+\eta$ where $f \cdot \eta$ is smooth if $f$ is a local defining equation of $V$. Then $R(\alpha)=[\theta \mid V]$, a closed form on $V-S$ with perhaps singularities on $S$, the singular locus of $V$.

Given any variety $V$ in a complex manifold $W$, we can asssociate to it a differential form $\lambda$, called its kernel, where $\lambda$ is locally summable on $W$, has singular support in $V$ and such that $d \lambda=1_{V}+\psi$ where $\psi$ is a smooth form on $W$ and $1_{V}$ is the current of integration over $V$, i.e., $1_{V}[\omega]=\int_{V-S} \omega$ (cf. Poly [22] for 
the existence of $\lambda$ ). For $V$ a hypersurface, $\lambda=\Sigma_{U} e_{U} d f_{U} / f_{U}$ is the kernel associated to $V$.

5.9 Conjecture. If $\alpha \in H^{*}(W-V)$ where $V$ is a variety of codim $q$ and $\lambda$ is the kernel of $V$, then $\alpha$ has a representative of the form $\omega=\theta \wedge \lambda+\eta$ where $\eta$ is smooth and $R(\alpha)=\theta \mid V$ which will be a closed form on $V-S$ with (perhaps) singularities on $S$, the singular locus of $V$.

Note. In general, $\operatorname{Res}(\alpha)=R(\alpha)$ is considered as a current with support in $V$, but what Conjecture 5.11 says is that $\operatorname{Res}(\alpha)$ can be represented by a closed differential form on $V$ but perhaps with singularities on the singular locus of $V$.

5.10 TheOREM (Poly). A necessary and sufficient condition that $\alpha \in$ $H^{p}(W-V)$ has a representative of the form $\omega=\theta \wedge \lambda+\eta$ where $\theta$ is smooth and $\theta \mid V$ is closed is that if $\alpha^{*}$ is the dual homology class of $\alpha$ via integration, then $\alpha^{*}$ has a representative of the form $\gamma_{p}=\tau\left(\gamma_{p-2 q+1}\right)$ where $0 \neq$ $\left[\gamma_{p-2 q+1}\right] \in H_{p-2 q+1}(V)$.

Proof. This is proven in [22] where it is stated slightly differently.

In Gordon [6], it is shown that $\bigcap[V]: H^{2 n-p-1}(V) \simeq H_{p-2 q+1}(V)_{\Delta}$ where $n=\operatorname{dim}_{\mathbf{C}} W$ and $\bigcap[V]$ is cap product with the homology class carried by $V$. But if $\beta \cap[V]=\left[\gamma_{p-2 q+1}\right] \in H_{p-2 q+1}(V)_{\Delta} \cap H_{p-2 q+1}(V)$, then $0 \neq$ $\delta * \beta \in H^{2 n-p}(W, V)$, i.e., we have the commutative diagram

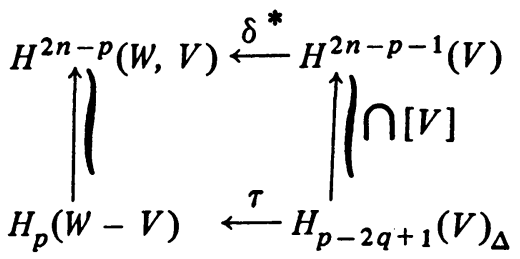

where the left-hand isomorphism is Lefschetz duality. Hence Theorem 5.12 is equivalent to:

$\alpha \in H^{p}(W-V)$ has a representative of the form $\theta \wedge \lambda+\eta$ where $\theta, \eta$ are smooth and $\lambda$ is kernel associated to $V$ if and only if we let $\alpha^{*} \in H_{2 n-p}(W, V)$ be the Lefschetz dual to $\alpha$, then $\delta_{*} \alpha^{*}=[V] \cap \beta$ for some $\beta \in H^{p-2 q+1}(V)$ and $\delta^{*} \alpha^{*} \in H_{2 n-p-1}(V)$. This is the form of the theorem that Poly proves. Q.E.D. for Theorem 5.10.

We note that a direct proof of Theorem 5.12 can be proved without use of currents in the theory of Bloom-Herrara and Herrera-Liberman [12]. The basic idea is that given $\gamma_{p-2 q+1}$, we can find a $(p-2 q+1)$-form $\theta$ on $W$ which is closed near $V$ such that $\theta$ is dual to $V$ to $\gamma_{p-2 q+1}$ via integration. Then one shows that $d(\theta \wedge \lambda)=d \xi$ for $\xi \in \Omega(W)$ by the fundamental identity

$$
\int_{\tau\left(\gamma_{p-2 q+1)}^{\prime}\right.} \theta \wedge \lambda=K \int_{\gamma_{p-2 q+1}^{\prime}} \theta \mid V \text { for } \gamma_{p-2 q+1}^{\prime} \in H_{p-2 q+1}(V)_{\Delta}
$$


and $K$ is some constant depending only on $q$. Then $\theta \wedge \lambda-i * \xi-i^{*} \rho \in \alpha$ for some $\rho \in \Omega(W)$.

We note that Robin [23] has shown that any $\alpha \in H^{*}(W-V)$ can be represented by a form with a pole of order $k$ for some $k$, but has no bounds on $k$. We can state

5.11 Corollary. Any class $\alpha \in H^{*}(W-V)$ has a representative $\omega$ such that $f^{2} \omega$ is continuous, where $f$ is a local defining equation.

Proof. Choose a resolution with normal crossings $\left(W^{\prime}, V^{\prime}, \pi\right)$ such that $f \circ \pi=f^{\prime}$ locally. But we can choose $\omega \in \alpha \in H^{*}(W-V)$ with $f^{\prime} \pi^{*} \omega=$ $\pi^{*} f \omega$ smooth. Hence for $P \in S, \pi^{-1}(P)$ is a compact set since $\pi$ is proper, so that $\left.\pi^{*} f \omega\right|_{\pi^{-1}(P)}<\infty$. Thus, $\lim _{Q \rightarrow P ; Q \in W-S} f^{2}(Q) \omega(Q)=0$, so that $f^{2} \omega$ is continuous. Q.E.D. for Corollary 5.11.

The question of semimeromorphic forms will be discussed in a subsequent paper.

I would like to conclude this paper by returning to Theorem 5.2 and consider the case when $\omega$ is a $(p, 0)$-form. This will tie up with the question asked in $\S 3$.

5.12 COROLlARY. If $V$ has normal crossings and $\omega$ is a $(p, 0)$-form with a pole of order one and $R(\omega) \in H^{p-q}\left(\bar{M}_{q}\right)_{\Delta}$, then there is a sufficiently small neighborhood $U_{q}$ of $\bar{M}_{q+1}$ in $W$ such that for all $\gamma_{p-q} \in H_{p-q}\left(V_{i_{1}} \cap \cdots \cap V_{i_{q}}\right)$,

$$
\int_{\gamma_{p-q}} R \omega=\int_{\gamma_{p-q} \cap\left(\bar{M}_{q}-U_{q}\right)} R \omega=\int_{\gamma_{p-q \cap M_{q}}} R \omega .
$$

Note. In general for $\omega$ a $(p, 0)$-form with a pole of order one, $R(\omega) \in$ $\sum_{q=1}^{p} H^{p-q}\left(\bar{M}_{q}\right)_{\Delta}$, i.e., $R(\omega)=\Sigma_{q} R_{q}(\omega)$ and the corollary will be true for each $R_{q}(\omega)$ and some $U_{q}$.

Proof. It suffices to assume $\omega$ is of the type

$$
\tilde{\theta} \wedge \sum_{U} e_{U} \frac{d f_{1, U}}{f_{1, U}} \wedge \ldots \wedge \frac{d f_{q, U}}{f_{q, U}}+\eta .
$$

Since $\eta$ is smooth and $d f_{i, U}$ is a $(1,0)$ form, this implies $\tilde{\theta}$ is a $(p-q, 0)$-form. Then let $\theta=R(\omega)=(2 \pi \sqrt{-1})^{q} \tilde{\theta} \mid \bar{M}_{q}$.

Let $\gamma_{p-q} \in H_{p-q}(\bar{M}(1, \ldots, q))$ and suppose $\gamma_{p-q} \cap \bar{M}_{q+1} \neq 0$ for all representatives of $\gamma_{p-q}$. Then we have $\gamma_{p-q} \cap V_{q+1}=\gamma_{p-q-2} \neq 0$ in $\bar{M}(1, \ldots, q, q+1)$.

Also,

$$
\int_{\gamma_{p-q}} \theta=\sum_{U \cap M_{q+1}=\varnothing} \int_{\gamma_{p-q} \cap U} e_{U} \cdot \theta+\sum_{U \cap M_{q+1} \neq \varnothing} \int_{\gamma_{p-q} \cap U} e_{U} \cdot \theta
$$

where $\{U\}$.is a cover of $W$ by coordinate charts.

To prove the corollary it suffices to show if $U \cap M_{q+1}=\varnothing$, then 
$\int_{\gamma_{p-q} \cap U} e_{U} \cdot \theta=0$. But this is so because in $U$, we have that

$$
\left.\gamma_{p-q} \cap U=\frac{(0 \times \cdots \times 0}{(q-1) \text {-times }} \times \mathrm{C}_{z_{q}} \times \gamma_{p-q-2}\right) \cap U
$$

since $\gamma_{p-q}$ intersects $V_{q+1}$ transversely. But $\theta$ is a $(p-q, 0)$-form, hence does not have a $d \bar{z}_{q}$-term which implies the integral must be zero. Q.E.D.

Note. The corollary does not say that if $\gamma_{p-q}$ is the dual cycle to $R(\omega)$ in $\bar{M}(1, \ldots, q)$ that $\gamma_{p-q}$ has a representative disjoint from $\bar{M}_{q+1}$. We can have $\gamma_{p-q} \cap V_{q+1}=\gamma_{p-q-2} \nsim 0$ in $\bar{M}(1, \ldots, q+1)$ but $\gamma_{p-q-2} \sim 0$ in $\bar{M}(1, \ldots, q)$. What this says is that the restriction of $R \omega$ to $U_{q} \cap \bar{M}_{q}$ is exact. However we have

5.13 CoRollary. Let $V$ have normal crossings and let $\omega$ be $a(p, 0)$-form with a pole of order one and $R(\omega) \in H^{p-q}\left(\bar{M}_{q}\right)_{\Delta}$. If in

$H_{p-q}(\bar{M}(1, \ldots, q)) \stackrel{I}{\rightarrow} H_{p-q-2}(\bar{M}(1, \ldots, q+1)) \stackrel{i_{*}}{\rightarrow} H_{p-q-2}(\bar{M}(1, \ldots, q))$

where $I$ is intersection and $i_{*}$ is induced from the inclusion, we have (image $I$ ) $\cap$ $\operatorname{Ker} i_{*}=0$, then $R(\omega) \in H^{p-q}\left(M_{q}\right)$, i.e., dual cycle to $R(\omega)$ lies in $H_{p-q}\left(M_{q}\right)$.

Proof. In the notation of Corollary 5.11, we have $\gamma_{p-q-2} \nsim 0$ in $H_{p-q-2}(\bar{M}(1, \ldots, q+1))$. But from the commutative diagram

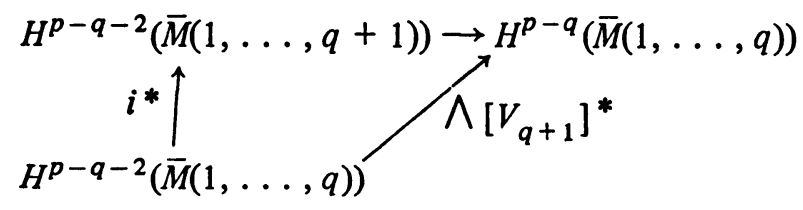

this implies that $\theta=\theta^{\prime} \wedge\left[V_{q+1}\right]^{*}$ where $\left[V_{q+1}\right]^{*}$ is the Poincare dual cycle to $V_{1} \cap \cdots \cap V_{q} \cap V_{q+1}$ in $\bar{M}(1, \ldots, q)$. But $\left[V_{q+1}\right]^{*}$ is a $(1,1)$-form which implies $\theta$ cannot be a $(p-q, 0)$-form contradiction. Q.E.D.

Note. If $V$ comes from a resolution, then the hypothesis that image $I \cap$ $\operatorname{Ker} i_{*}=0$ may always be true.

We know that if $V$ is ample, then $H^{p}(W-V)$ is generated by meromorphic $(p, 0)$-forms with poles of order $k \leqslant n=\operatorname{dim}_{\mathrm{C}} W$. Griffiths [8, §8] has given an algorithm for representing $\omega=\sum_{i=0}^{k-1} \omega_{i}$ where $\omega_{i}$ has a pole of order one and $\omega_{i}$ is a $(p-i, i)$-form. Using the fact that $\omega$ is meromorphic, one might be able to generalize Corollary 5.12 without too restrictive topological conditions on $V$ which are satisfied if $V$ is a resolution.

\section{BIBLIOGRAPHY}

1. A. Andreotti and T. Frankel, The Lefschetz theorem on hyperplane sections, Ann. of Math. (2) 69 (1959), 713-717.

2. C. H. Clemens, Jr., Picard-Lefschetz theorem for families of nonsingular algebraic 
varieties acquiring ordinary singularities, Trans. Amer. Math. Soc. 136 (1969), 93-108. MR 38 \#2135.

3. R. Courant and D. Hilbert, Methods of mathematical physics. Vol. II: Partial differential equations, Interscience, New York, 1962. MR 25 \#4216.

4. P. Deligne, Théorie de Hodge. II, Inst. Hautes Études Sci. Publ. Math. No. 40 (1972), 5-57.

5. G. L. Gordon, Differentials of the second kind (to appear).

6. - A Poincaré type theorem for polyhedra, Ann. Inst. Fourier (Grenoble) 22 (1972), 47-58.

7. P. A. Griffiths, Periods of integrals on algebraic manifolds: Summary of main results and discussion of open problems, Bull. Amer. Math. Soc. 76 (1970), 228-296. MR 41 \#3470.

8. P. A. Griffiths, On the periods of certain rational integrals. I, Ann. of Math. (2) 90 (1969), 460-495. MR 14 \#5357.

9. H. Hironaka, Resolution of singularities of an algebraic variety over a field of characteristic zero. I, II, Ann. of Math. (2) 79 (1964), 109-203, 205-326. MR 33 \#7333.

10. - Sub-analytic sub-sets (to appear).

11. W. V. D. Hodge and M. F. Atiyah, Integrals of the second kind on an algebraic variety, Ann. of Math. (2) 62 (1955), 56-91. MR 17, 533.

12. D. Liberman and $M$. Herrera, Residues and principle values on complex spaces, Math. Ann. 194 (1971), 259-294.

13. M. Lejeune and B. Teissier, Quelques calculs utiles pour la résolution des singularitiés, Seminar at École Polytechnique, 1972.

14. J. Leray, Le calcul différentiel et intégral sur une variété analytique complexe (Problème de Cauchy. III), Bull. Soc. Math. France 87 (1959), 81-180. MR 23 \#A3281.

15. J. Milnor, Differential structures, Mimeographed notes, Princeton, 1961.

16. Differentiable manifolds which are homotopy spheres, Mimeographed notes, Princeton, 1959.

17. - Singular points of complex hypersurfaces, Ann. of Math. Studies, no. 61, Princeton Univ. Press, Princeton, N.J., Univ. of Tokyo Press, Tokyo, 1968. MR 39 \#969.

18. B. G. Moǐsezon, Algebraic homology classes on algebraic varieties, Izv. Akad. Nauk SSSR Ser. Mat. 31 (1967), 225-268 = Math. USSR Izv. 1 (1967), 209-251. MR 35 \#4215.

19. D. Mumford, The topology of normal singularities of an algebraic surface and a criterion for simplicity, Inst. Hautes Ėtudes Sci. Publ. Math. No. 9 (1961), 5-22. MR 27 \#3643.

20. P. Orlik and P. Wagreich, Isolated singularities of algebraic surfaces with $C$ *actions, Ann. of Math. 93 (1971), 205-228.

21. F. Pham, Formules de Picard-Lefschetz généralisées et ramification des intégrales,

Bull. Soc. Math. France 93 (1965), 333-367. MR 33 \#4064.

22. J. B. Poly, Sur un théoreme de J. Leray en théorie des résidues (to appear).

23. G. Robin, Formes semi-méromorphes et cohomologie du complémentaire d'une hypersurface d'une variété analytique complexe, C. R. Acad. Sci. Paris Sér. A-B 272 (1971), A33-A35. MR 44 \#476.

24. R. Thom, Ensembles et morphismes stratifiés, Bull. Amer. Math. Soc. 75 (1969), 240-284. MR 39 \#970.

25. P. Wagreich, Elliptic singularities of surfaces, Amer. J. Math. 92 (1970), 419-454. MR 45 \#264.

26. A. Weil, Introduction à l'étude des variétés kähleriennes, Publ. Inst. Math. Univ. Nancago, VI, Actualités Sci. Ind., no. 1267, Hermann, Paris, 1958. MR 22 \#1921.

27. H. Whitney, Local properties of analytic varieties, Differential and Combinatorial Topology (A Sympos. in Honor of Marston Morse), Princeton Univ. Press, Princeton, N.J., 1965, pp. 205-244. MR $32 \# 5924$.

28. O. Zariski, Algebraic surfaces, Ergebnisse der Mathematik und ihrer Grenzgebiete, Band 3, Springer-Verlag, Berlin, 1935. 